\author{
UNIVERSIDADE DE SÃO PAULO \\ INSTITUTO DE QUÍMICA DE SÃO CARLOS \\ PROGRAMA DE PÓS-GRADUAÇÃO EM QUÍMICA
}

TEORIA DO FUNCIONAL DA DENSIDADE APLICADA AO ESTUDO DA INTERAÇÃO ENTRE OXIGÊNIO MOLECULAR E NANOCLUSTERS DE PLATINA DOPADOS COM Al, Cr e V.

Jaldyr de Jesus Gomes Varela Júnior

São Carlos 


\author{
UNIVERSIDADE DE SÃO PAULO \\ INSTITUTO DE QUÍMICA DE SÃO CARLOS \\ PROGRAMA DE PÓS-GRADUAÇÃO EM QUÍMICA
}

\title{
TEORIA DO FUNCIONAL DA DENSIDADE APLICADA AO ESTUDO DA INTERAÇÃO ENTRE OXIGÊNIO MOLECULAR E NANOCLUSTERS DE PLATINA DOPADOS COM Al, Cr e V.
}

\author{
Jaldyr de Jesus Gomes Varela Júnior \\ Orientador: Prof. Dr. Albérico Borges Ferreira da Silva \\ Tese Apresentada ao Instituto de Química de \\ São Carlos, da Universidade de São Paulo, \\ para a obtenção do título de Doutor em \\ Ciências - Área de Concentração: Físico \\ Química.
}

São Carlos 


\section{TEORIA DO FUNCIONAL DA DENSIDADE APLICADA AO ESTUDO DA INTERAÇÃO ENTRE OXIGÊNIO MOLECULAR E NANOCLUSTERS DE PLATINA DOPADOS COM Al, Cr e V.}

Jaldyr de Jesus Gomes Varela Júnior

Aprovado em: I I

BANCA EXAMINADORA:

Prof. Dr. Albérico Borges Ferreira da Silva (Orientador)
DFQM - IQSC - USP

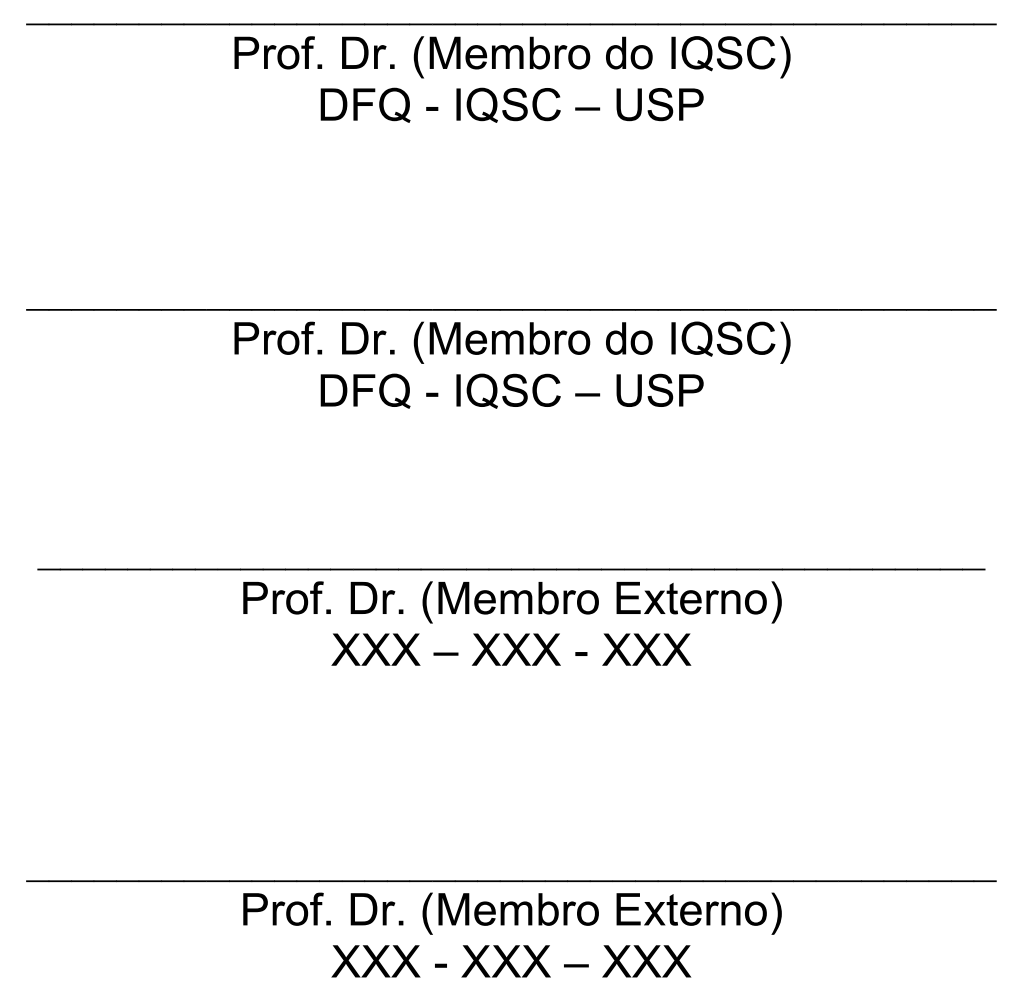


Você não sabe o quanto eu caminhei

Pra chegar até aqui

Percorri milhas e milhas

Antes de dormir, eu não cochilei

Os mais belos montes escalei

$\mathcal{N}$ as noites escuras de frio chorei..

(Toni Garrido)

O que você fizer será insignificante, mas é da maior importância que o faça.

(Mahatma Gandhi) 
Dedico este trabalho

Às minhas mães Maria da Paz F. Costa e Ildener Sousa Costa pela extrema dedicação, exemplo de vida e por terem sempre acreditado e confiado em mim.

Aos meus tios Boaventura, Benedito, Mário, Santos, Balbina Cristina, $\mathcal{M}^{a}$ do Socorro e Roseli pelo incentivo e ajuda quando solicitados.

Aos meus Irmãos Ennos e Débora e ao Gabriel pelo carinho. 
Ao Prof. Dr. Albérico Borges Ferreira da Silva, meu especial agradecimento, por sua valiosa orientação $e$ acima de tudo, pela paciência, amizade e incentivos sempre que foi solicitado. 


\section{AGRADECIMENTOS}

Agradeço primeiramente a Deus, por ter me dado a oportunidade de estar aqui neste momento, crescendo e aprendendo.

Ao Prof. Dr. Auro A. Tanaķa (DEQVI - VFMA) pelo espaço, amizade e pelo incansável suporte.

Ao Prof. Dr. Francisco Carlos $\mathcal{N a r t}$ (em memória) pelos ensinamentos, apoio e atenção dispensada durante os meus primeiros dias na cidade de São Carlos.

Ao Prof. Dr. Francisco das Chagas Alves Lima (DQ-UESPI) pela ajuda no inicio do desenvolvimento desse trabalho.

Ao Prof. Dr. Edson A. Ticianelli pela oportunidade, apoio e orientação nos meus primeiros anos de curso.

Aos amigos Adilson Luís Pereira Silva e Manuel de Jesus Santiago Farias pelas valiosas discussões, sugestões e convivência agradável durante esses anos.

Aos Alunos e Colegas Professores do Colégio Universitário da Universidade Federal do Maranhão pela convivência agradável e momentos de discussões sobre a escola, astronomia, ciências, música, etc.

Aos Colegas da Pós Graduação do IQSC: Rommel, Michael (Gorda), Benedito (Bibi), Vlisses, Zé Luis, Baixinho, Magão, Lenilson (Pho6s), Hércules, Ana Fornazari, Scooby, Tarzan, Roberto, Bruno e MMelke pelos momentos de discussões e convivência.

A todos os colegas do Laboratório de Eletroquímica da UFMA pela oportunidade de ter trabalhado com todos.

Aos Colegas do IFMA / CAMPUS - SANTA INNÊS pela convivência agradável e momentos de discussões sobre a escola e conhecimentos científicos.

À FAPEMA pela 6olsa concedida e pelo apoio financeiro concedido para realização de uma parte desta pesquisa (Edital Universal/Fapema $\mathcal{N}^{\circ} 10 / 2009$ ). 


\begin{tabular}{|c|c|}
\hline SUMÁRIO & \\
\hline 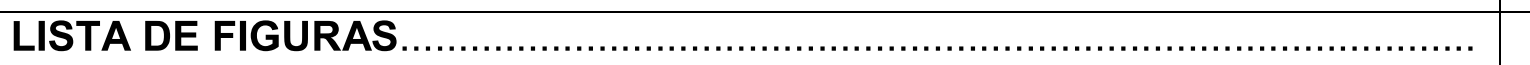 & 1 \\
\hline LISTA DE TABELAS & iii \\
\hline RESUMO & $\mathrm{v}$ \\
\hline ABSTRACT. & vi \\
\hline 1. INTRODUÇÃO & 1 \\
\hline 1.1 A Reação de Redução de Oxigênio. & 1 \\
\hline 1.2 A Teoria do Funcional da Densidade (DFT) & 7 \\
\hline \begin{tabular}{|l|l|} 
1.3 Estudos Teóricos da Reação de Redução de Oxigênio......................... & 1
\end{tabular} & 19 \\
\hline 2. OBJETIVO & 24 \\
\hline 3. DETALHES COMPUTACIONAIS & 25 \\
\hline 4. RESULTADOS E DISCUSSÃO & 28 \\
\hline $\begin{array}{l}\text { 4.1 Otimização e Propriedades Eletrônicas dos Clusters } \mathbf{P t}_{2} \text {, Pt-M e } \mathbf{M}_{\mathbf{2}} \\
\text { (M=Al, } \mathrm{Cr} \text { e V) }\end{array}$ & 28 \\
\hline $\begin{array}{l}4.2 \text { Otimização e Propriedades Eletrônicas dos Clusters } \mathbf{P t}_{3} \text { e } \mathbf{P t}_{2} \mathbf{M} \\
(\mathrm{M}=\mathrm{Al}, \mathrm{Cr} \text { e V) }\end{array}$ & 31 \\
\hline $\begin{array}{l}\text { 4.3 Otimização e Propriedades Eletrônicas dos Clusters } \mathbf{P t}_{4} \text { e } \mathbf{P t}_{3} \mathbf{M} \\
(\mathrm{M}=\mathrm{Al}, \mathrm{Cr} \text { e V) }\end{array}$ & 34 \\
\hline
\end{tabular}




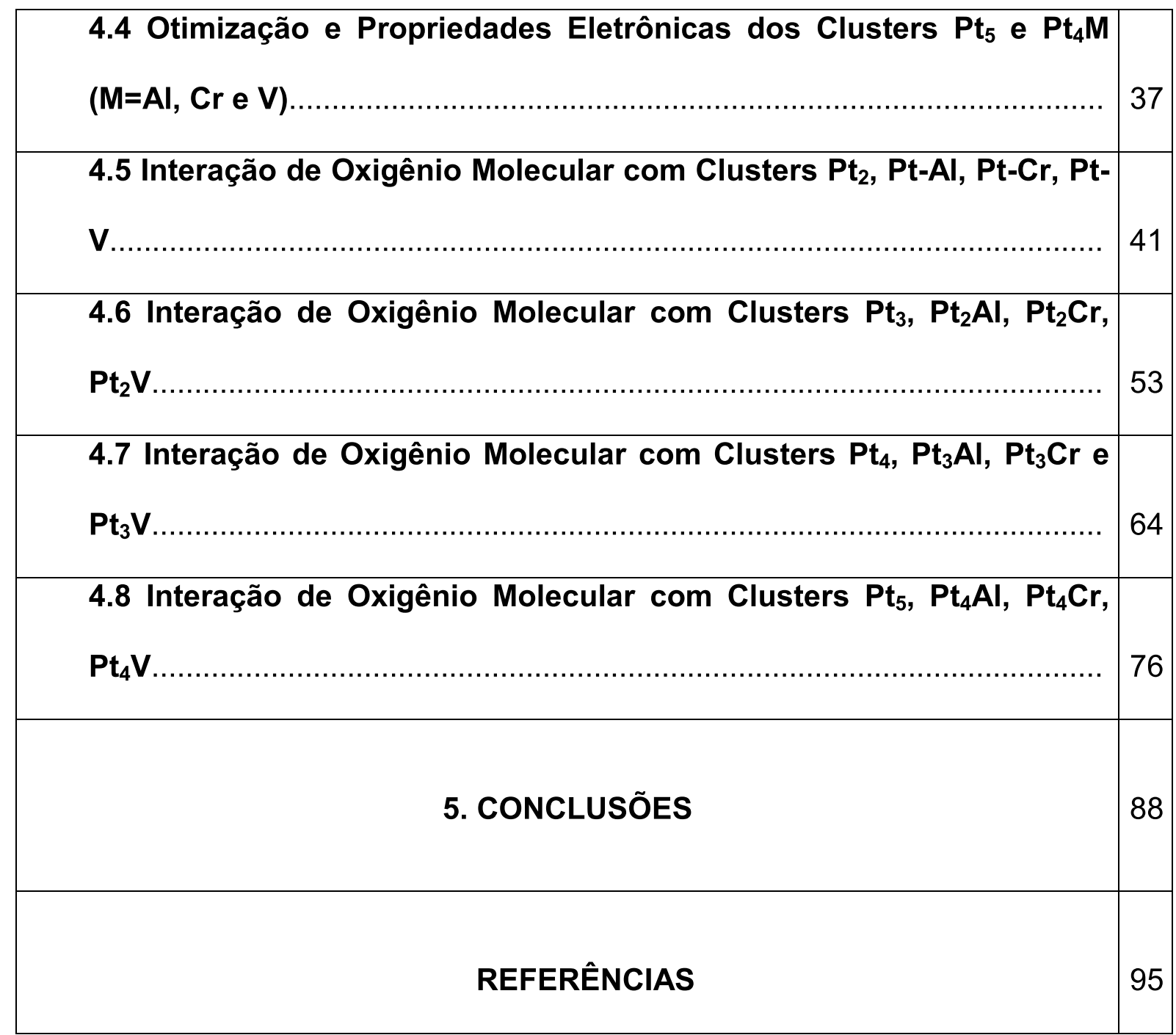




\begin{tabular}{|c|c|c|}
\hline & LISTA DE FIGURAS & \\
\hline FIGURA 1: & Modelos de adsorção de oxigênio na superfície do eletrodo......... & 3 \\
\hline FIGURA 2: & Possíveis rotas reacionais para a RRO em soluções ácidas......... & 4 \\
\hline FIGURA 3: & Esquema representativo do ciclo de autoconsistência...................... & 15 \\
\hline FIGURA 4: & $\begin{array}{l}\text { Estruturas usadas como entrada (input) dos cálculos de } \\
\text { otimização dos nano-clusters } \mathrm{Pt}_{3}, \mathrm{Pt}_{4}{\mathrm{e} \mathrm{Pt}_{5} \ldots \ldots \ldots \ldots \ldots \ldots \ldots \ldots \ldots \ldots}\end{array}$ & 26 \\
\hline FIGURA 5: & 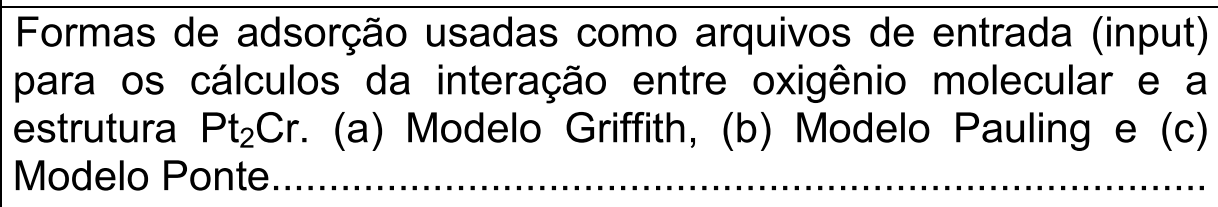 & 27 \\
\hline FIGURA 6: & $\begin{array}{l}\text { Geometrias das estruturas }(\mathrm{a}) \mathrm{Pt}_{4},(\mathrm{~b}) \mathrm{Pt}_{3} \mathrm{Al}, \text { (c) } \mathrm{Pt}_{3} \mathrm{Cr} \text { e (d) } \mathrm{Pt}_{3} \mathrm{~V} \\
\text { otimizadas com o método } \mathrm{B} 3 \mathrm{~L} Y \mathrm{P} / \mathrm{LANL} 2 \mathrm{DZ} \ldots \ldots \ldots \ldots \ldots \ldots \ldots \ldots \ldots\end{array}$ & 35 \\
\hline FIGURA 7: & $\begin{array}{l}\text { Geometrias das estruturas (a) } \mathrm{Pt}_{5},(\mathrm{~b}) \mathrm{Pt}_{4} \mathrm{Al}, \text { (c) } \mathrm{Pt}_{4} \mathrm{Cr} \text { e (d) } \mathrm{Pt}_{4} \mathrm{~V} \\
\text { otimizadas com o método } \mathrm{B} 3 \mathrm{~L} \mathrm{YP} / \mathrm{LANL} 2 \mathrm{DZ} \ldots \ldots \ldots \ldots \ldots \ldots \ldots \ldots \ldots \ldots\end{array}$ & 39 \\
\hline FIGURA 8: & 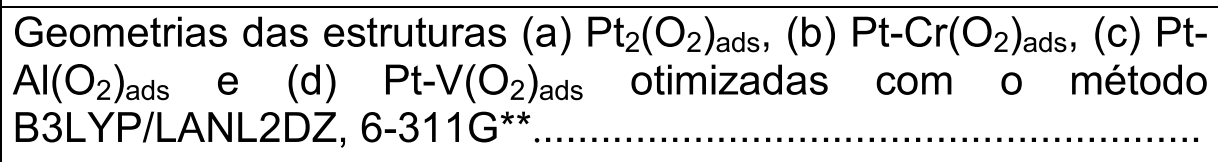 & 43 \\
\hline FIGURA 9: & 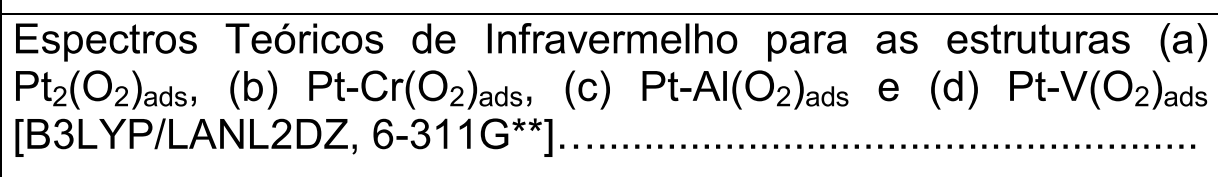 & 43 \\
\hline FIGURA 10: & 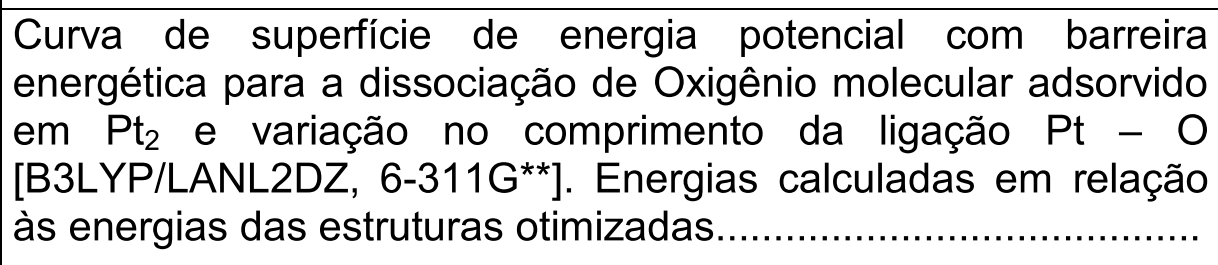 & 51 \\
\hline FIGURA 11: & $\begin{array}{l}\text { Curva de superfície de energia potencial com barreira } \\
\text { energética para a dissociação de Oxigênio molecular adsorvido } \\
\text { em } \mathrm{Pt}_{2}, \mathrm{Pt}-\mathrm{Cr} \text { e } \mathrm{Pt}-\mathrm{V} \text { [B3LYP/LANL2DZ, 6-311G**]. Energias } \\
\text { calculadas em relação às energias das estruturas otimizadas..... }\end{array}$ & 52 \\
\hline FIGURA 12: & 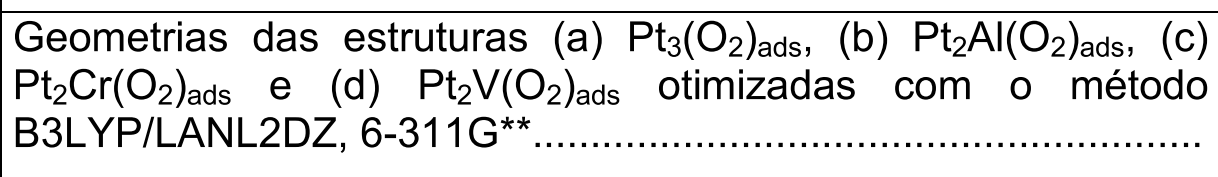 & 54 \\
\hline FIGURA 13: & Espectros Teóricos de Infravermelho para as estruturas (a) & \\
\hline
\end{tabular}




\begin{tabular}{|c|c|c|}
\hline & $\begin{array}{l}\mathrm{Pt}_{3}\left(\mathrm{O}_{2}\right)_{\text {ads }}, \text { (b) } \mathrm{Pt}_{2} \mathrm{Al}\left(\mathrm{O}_{2}\right)_{\text {ads, }} \text { (c) } \mathrm{Pt}_{2} \mathrm{Cr}\left(\mathrm{O}_{2}\right)_{\text {ads }} \text { e (d) } \mathrm{Pt}_{2} \mathrm{~V}\left(\mathrm{O}_{2}\right)_{\text {ads }} \\
\text { [B3LYY/LANL2DZ, 6-311G** }\end{array}$ & 55 \\
\hline FIGURA 14: & $\begin{array}{l}\text { Curva de superfície de energia potencial com barreira } \\
\text { energética para a dissociação de oxigênio molecular adsorvido } \\
\text { em } \mathrm{Pt}_{2} \mathrm{Al} \text { e } \mathrm{Pt}_{2} \mathrm{~V} \quad\left[\mathrm{~B} 3 \mathrm{LYP} / \mathrm{LANL} 2 \mathrm{DZ}, \quad 6-311 \mathrm{G}^{* *}\right] \text {. Energias } \\
\text { calculadas em relação às energias das estruturas otimizadas..... }\end{array}$ & 62 \\
\hline FIGURA 15: & 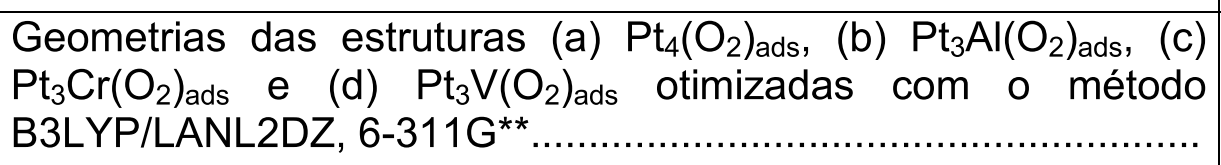 & 65 \\
\hline FIGURA 16: & $\begin{array}{l}\text { Espectros Teóricos de Infravermelho para as estruturas (a) } \\
\mathrm{Pt}_{4}\left(\mathrm{O}_{2}\right)_{\text {ads, }}(\mathrm{b}) \mathrm{Pt}_{3} \mathrm{Al}\left(\mathrm{O}_{2}\right)_{\text {ads, }} \text { (c) } \mathrm{Pt}_{3} \mathrm{Cr}\left(\mathrm{O}_{2}\right)_{\text {ads }} \text { e (d) } \mathrm{Pt}_{3} \mathrm{~V}\left(\mathrm{O}_{2}\right)_{\text {ads }} \\
\text { [B3LYP/LANL2DZ, 6-311G*] }\end{array}$ & 66 \\
\hline FIGURA 17: & $\begin{array}{l}\text { Curva de superfície de energia potencial com barreira } \\
\text { energética para a dissociação de oxigênio molecular adsorvido } \\
\text { em } \mathrm{Pt}_{3} \mathrm{Al} \text { e } \mathrm{Pt}_{3} \mathrm{~V} \quad\left[\mathrm{~B} 3 \mathrm{LYP} / \mathrm{LANL} 2 \mathrm{DZ}, \quad 6-311 \mathrm{G}^{* *}\right] \text {. Energias } \\
\text { calculadas em relação às energias das estruturas otimizadas.... }\end{array}$ & 75 \\
\hline FIGURA 18: & 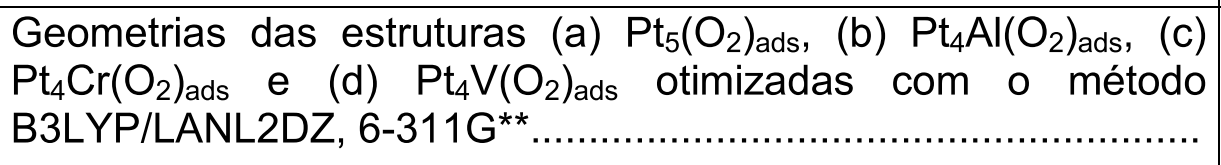 & 77 \\
\hline FIGURA 19: & 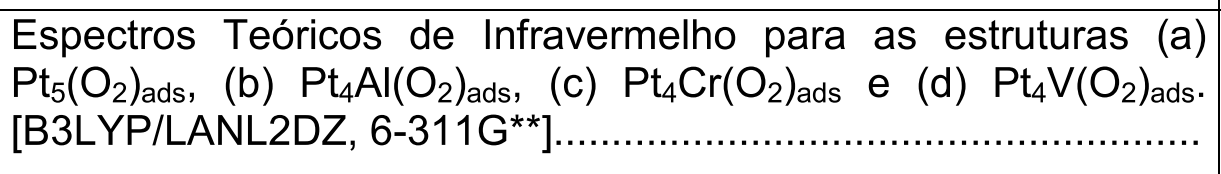 & 79 \\
\hline FIGURA 20: & $\begin{array}{l}\text { Curva de superfície de energia potencial com barreira } \\
\text { energética para a dissociação de oxigênio molecular adsorvido } \\
\text { em } \mathrm{Pt}_{4} \mathrm{Al} \text { e } \mathrm{Pt} \mathrm{t} \mathrm{V} \quad\left[\mathrm{B} 3 \mathrm{LYP} / \mathrm{LANL} 2 \mathrm{DZ}, \quad 6-311 \mathrm{G}^{\star *}\right] \text {. Energias } \\
\text { calculadas em relação às energias das estruturas otimizadas..... }\end{array}$ & 86 \\
\hline
\end{tabular}




\begin{tabular}{|c|c|c|}
\hline & LISTA DE TABELAS & \\
\hline Tabela 1: & $\begin{array}{l}\text { Geometrias otimizadas, propriedades energéticas e multiplicidade } \\
\text { de spin para os clusters } \mathrm{Pt}_{2}, \mathrm{Al}_{2}, \mathrm{Cr}_{2}, \mathrm{~V}_{2}, \mathrm{Pt}-\mathrm{Al}, \mathrm{Pt}-\mathrm{Cr} \text { e Pt-V. } \\
\text { Método B3LYP/LANL2DZ }\end{array}$ & 29 \\
\hline Tabela 2: & $\begin{array}{l}\text { Cargas atômicas de Mulliken e configurações eletrônicas dos } \\
\text { átomos presentes nos clusters heteronucleares } \mathrm{Pt}-\mathrm{Al}, \mathrm{Pt}-\mathrm{Cr} \text { e Pt-V. }\end{array}$ & 30 \\
\hline Tabela 3: & $\begin{array}{l}\text { Distâncias interatômicas, propriedades energéticas, multiplicidade } \\
\text { de spin, cargas atômicas de Mulliken e configurações eletrônicas } \\
\text { dos átomos presentes nos clusters } \mathrm{Pt}_{3}, \mathrm{Pt}_{2} \mathrm{Al}_{1}, \mathrm{Pt}_{2} \mathrm{Cr}_{\text {e }} \mathrm{Pt}_{2} \mathrm{~V} \text {...................... }\end{array}$ & 32 \\
\hline Tabela 4: & $\begin{array}{l}\text { Distâncias interatômicas, propriedades energéticas, multiplicidade } \\
\text { de spin, cargas atômicas de Mulliken e configurações eletrônicas } \\
\text { dos átomos presentes nos clusters } \mathrm{Pt}_{4}, \mathrm{Pt}_{3} \mathrm{Al}_{1}, \mathrm{Pt}_{3} \mathrm{Cr}_{\mathrm{a}} \text { e } \mathrm{Pt}_{3} \mathrm{~V} \ldots \ldots \ldots \ldots \ldots . . . . . . . . . .\end{array}$ & 35 \\
\hline Tabela 5: & 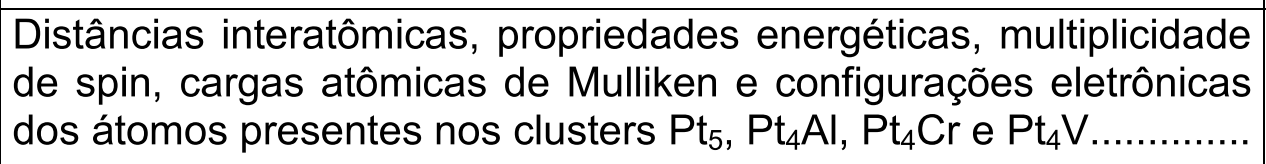 & 40 \\
\hline Tabela 6: & 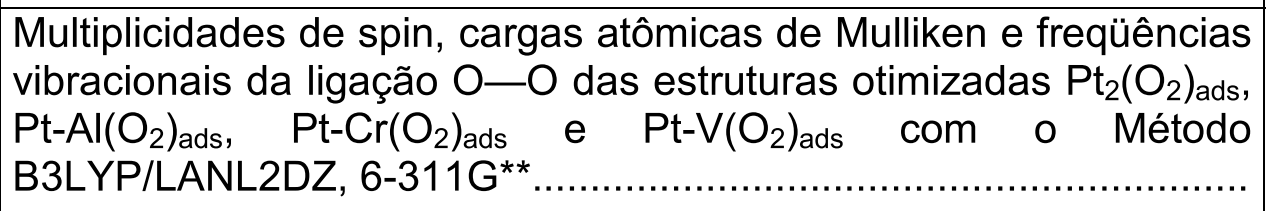 & 44 \\
\hline Tabela 7: & 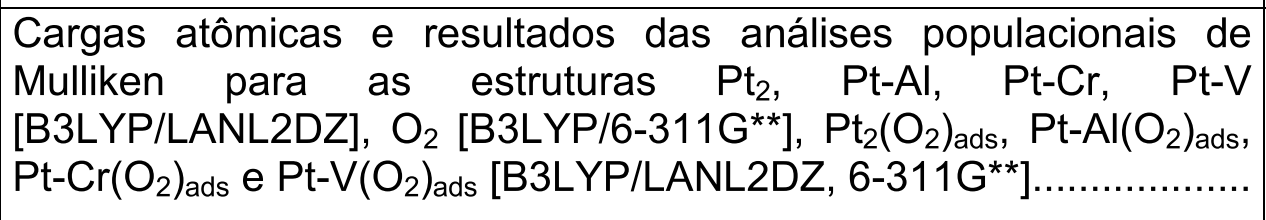 & 47 \\
\hline Tabela 8: & 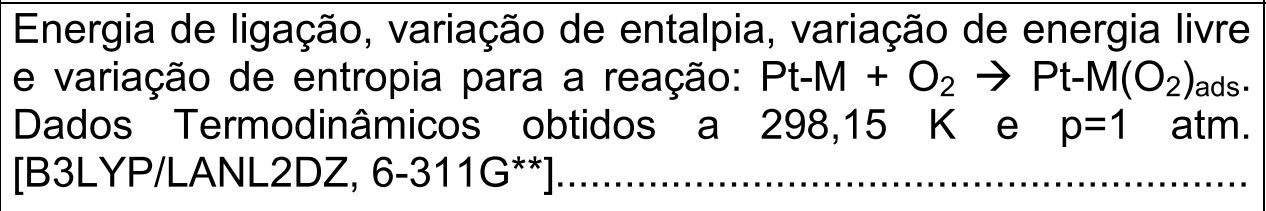 & 50 \\
\hline Tabela 9: & 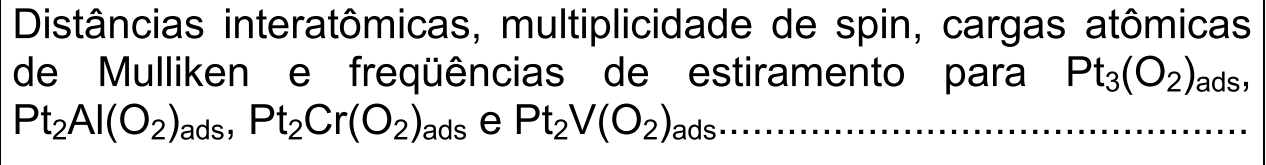 & 56 \\
\hline Tabela 10: & $\begin{array}{l}\text { Cargas atômicas e resultados das análises populacionais de } \\
\text { Mulliken para as estruturas } \mathrm{Pt}_{3}, \quad \mathrm{Pt}_{2} \mathrm{Al}, \quad \mathrm{Pt}_{2} \mathrm{Cr}, \quad \mathrm{Pt}_{2} \mathrm{~V} \\
\text { [B3LYP/LANL2DZ], } \mathrm{O}_{2}\left[\mathrm{~B} 3 \mathrm{LYP} / 6-311 \mathrm{G}^{* *}\right], \mathrm{Pt}_{3}\left(\mathrm{O}_{2}\right)_{\text {ads }}, \mathrm{Pt}_{2} \mathrm{Al}\left(\mathrm{O}_{2}\right)_{\text {ads }} \text {, } \\
\mathrm{Pt}_{2} \mathrm{Cr}\left(\mathrm{O}_{2}\right)_{\mathrm{ads}} \text { e } \mathrm{Pt} \mathrm{t}_{2} \mathrm{~V}\left(\mathrm{O}_{2}\right)_{\text {ads }}\left[\mathrm{B} 3 \mathrm{LYP} / \mathrm{LANL} 2 \mathrm{DZ}, 6-311 \mathrm{G}^{* *}\right] \ldots \ldots \ldots \ldots \ldots \ldots \ldots .\end{array}$ & 60 \\
\hline Tabela 11: & $\begin{array}{l}\text { Energia de ligação, variação de entalpia, variação de energia livre } \\
\text { e variação de entropia para a reação: } \mathrm{Pt}_{2} \mathrm{M}+\mathrm{O}_{2} \rightarrow \mathrm{Pt}_{2} \mathrm{M}\left(\mathrm{O}_{2}\right)_{\text {ads. }}\end{array}$ & \\
\hline
\end{tabular}




\begin{tabular}{|c|c|c|}
\hline & 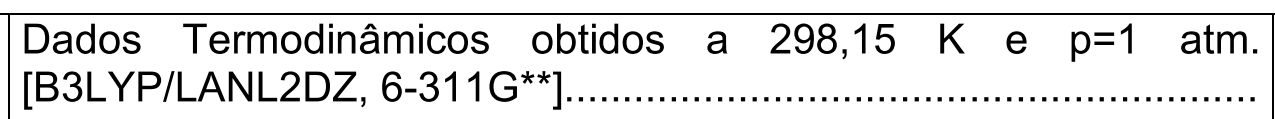 & 63 \\
\hline Tabela 12: & 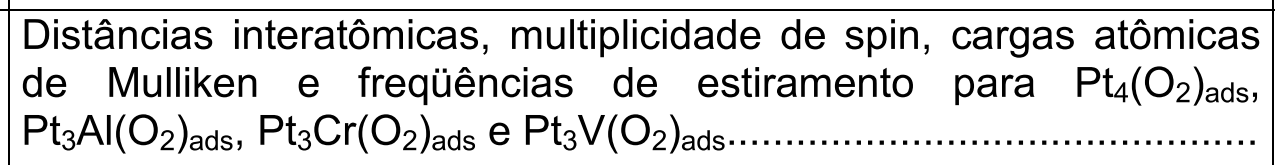 & 67 \\
\hline Tabela 13: & 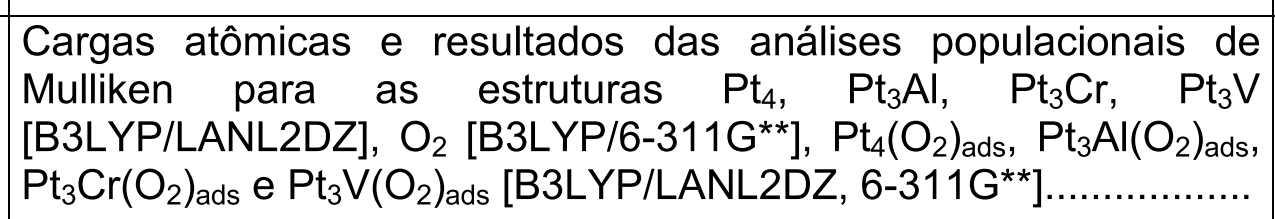 & 72 \\
\hline Tabela 14: & 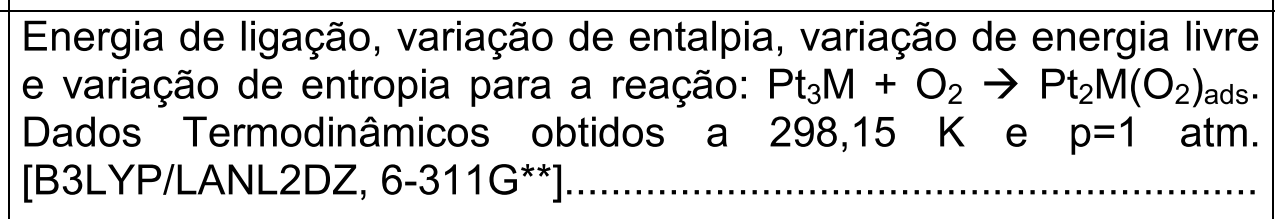 & 75 \\
\hline Tabela 15: & 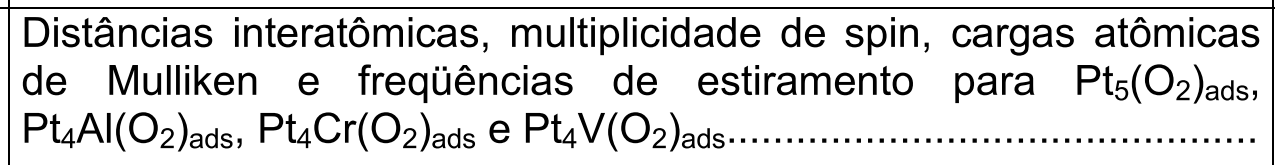 & 78 \\
\hline Tabela 16: & $\begin{array}{l}\text { Cargas atômicas e resultados das análises populacionais de } \\
\text { Mulliken para as estruturas } \mathrm{Pt}_{5}, \quad \mathrm{Pt}_{4} A \mathrm{Al}, \mathrm{Pt}_{4} \mathrm{Cr}, \mathrm{Pt}_{4} \mathrm{~V} \\
{[\mathrm{~B} 3 \mathrm{LYP} / \mathrm{LANL} 2 \mathrm{DZ}], \mathrm{O}_{2}\left[\mathrm{~B} 3 \mathrm{LYP} / 6-311 \mathrm{G}^{* *}\right], \mathrm{Pt}_{5}\left(\mathrm{O}_{2}\right)_{\text {ads, }}, \mathrm{Pt}_{4} \mathrm{Al}\left(\mathrm{O}_{2}\right)_{\text {ads }} \text {, }} \\
\mathrm{Pt}_{4} \mathrm{Cr}\left(\mathrm{O}_{2}\right)_{\text {ads }} \text { e } \mathrm{Pt} \mathrm{V}\left(\mathrm{O}_{2}\right)_{\text {ads }}\left[\mathrm{B} 3 \mathrm{LYP} / \mathrm{LANL} 2 \mathrm{DZ}, 6-311 \mathrm{G}^{\star *}\right] \ldots \ldots \ldots \ldots \ldots \ldots \ldots .\end{array}$ & 83 \\
\hline \begin{tabular}{|l} 
Tabela 17: \\
\end{tabular} & $\begin{array}{l}\text { Energia de ligação, variação de entalpia, variação de energia livre } \\
\text { e variação de entropia para a reação: } \mathrm{Pt}_{4} \mathrm{M}+\mathrm{O}_{2} \rightarrow \mathrm{Pt}_{4} \mathrm{M}\left(\mathrm{O}_{2}\right)_{\text {ads }} \text {. } \\
\text { Dados Termodinâmicos obtidos a } 298,15 \mathrm{~K} \text { e } \mathrm{p}=1 \text { atm. } \\
\left.\text { [B3LYP/LANL2DZ, } 6-311 \mathrm{G}^{\star *}\right] \ldots \ldots \ldots \ldots \ldots \ldots \ldots \ldots\end{array}$ & 87 \\
\hline
\end{tabular}




\section{Resumo}

Este trabalho apresenta um estudo aplicando a Teoria do Funcional da Densidade (DFT), utilizando o funcional B3LYP, para estudar a estrutura eletrônica de nanoclusters de platina dopados com $\mathrm{Al}, \mathrm{Cr}$ e $\mathrm{V}$ e suas interações com oxigênio molecular. As análises das populações de Mulliken e de NBO para a interação entre $\mathrm{O}_{2}$ e $\mathrm{Pt}_{2}$, Pt-Cr e Pt-V revelaram que ocorre transferência de carga dos orbitais $\mathrm{s}$ e d dos metais para os orbitais $p$ do oxigênio, resultando no preenchimento dos orbitais antiligantes da molécula de oxigênio, provocando a quebra da ligação $\mathrm{O}-\mathrm{O}$ e formação de ligações hibridizadas Metal - O, com energia de dissociação da ligação $\mathrm{O}$ - O em 1,0 eV sobre $\mathrm{Pt}_{2}$. Sobre Pt-Cr e Pt-V, esse valor decresce para 0,56 eV e $0,20 \mathrm{eV}$, respectivamente. Os estudos da interação entre oxigênio molecular e os clusters $\mathrm{Pt}_{3}, \mathrm{Pt}_{2} \mathrm{Al}, \mathrm{Pt}_{2} \mathrm{Cr}$ e $\mathrm{Pt}_{2} \mathrm{~V}$ mostram que ocorre adsorção não dissociativa de oxigênio molecular sobre o cluster $\mathrm{Pt}_{3}$ onde observamos uma adsorção segundo o modelo de ponte enquanto que sobre os clusters $\mathrm{Pt}_{2} \mathrm{Al}$ e $\mathrm{Pt}_{2} \mathrm{~V}$ ocorre adsorção dissociativa de oxigênio molecular. Por outro lado, com o cluster $\mathrm{Pt}_{2} \mathrm{Cr}$ a adsorção segue o modelo de Pauling, com o oxigênio molecular adsorvido em apenas um sítio do cluster, que foi o átomo $\mathrm{Pt}$, sem a quebra da ligação $\mathrm{O}-\mathrm{O}$. Curvas de superfície de energia potencial para a dissociação de oxigênio molecular sobre $\mathrm{Pt}_{2} \mathrm{Al}_{\mathrm{l}}$ e $\mathrm{Pt}_{2} \mathrm{~V}$ mostraram um valor de aproximadamente $0,21 \mathrm{eV}$ para a barreira de dissociação da ligação $\mathrm{O}-\mathrm{O}$ adsorvido sobre o cluster $\mathrm{Pt}_{2} \mathrm{Al}$ e aproximadamente $0,30 \mathrm{eV}$ para a barreira de dissociação da ligação $\mathrm{O}-\mathrm{O}$ adsorvido sobre o cluster $\mathrm{Pt}_{2} \mathrm{~V}$. Os estudos da interação entre oxigênio molecular e os clusters $\mathrm{Pt}_{4}, \mathrm{Pt}_{3} \mathrm{Al}, \mathrm{Pt}_{3} \mathrm{Cr}$ e $\mathrm{Pt}_{3} \mathrm{~V}$ mostram que ocorre adsorção não dissociativa de oxigênio molecular sobre os clusters $\mathrm{Pt}_{4} \mathrm{e}$ $\mathrm{Pt}_{3} \mathrm{Cr}$ onde foi observado que a adsorção segue o modelo de ponte. Por outro lado, sobre os clusters $\mathrm{Pt}_{3} \mathrm{Al}$ e $\mathrm{Pt}_{3} \mathrm{~V}$ a adsorção de oxigênio molecular também seguiu o modelo ponte, com dissociação da ligação $\mathrm{O}-\mathrm{O}$. Encontramos um valor de 0,46 eV para a barreira de dissociação da ligação $\mathrm{O}-\mathrm{O}$ adsorvido sobre o cluster $\mathrm{Pt}_{3} \mathrm{Al}$ e aproximadamente $0,28 \mathrm{eV}$ para a barreira energética de dissociação da ligação $\mathrm{O}$ $\mathrm{O}$ adsorvido sobre o cluster $\mathrm{Pt}_{3} \mathrm{~V}$. Os estudos para interação entre oxigênio molecular e os clusters $\mathrm{Pt}_{5}, \mathrm{Pt}_{4} \mathrm{Al}, \mathrm{Pt}_{4} \mathrm{Cr}$ e $\mathrm{Pt}_{4} \mathrm{~V}$ revelaram que ocorre adsorção dissociativa de oxigênio molecular sobre os clusters $\mathrm{Pt}_{4} \mathrm{Al}$ e $\mathrm{Pt}_{4} \mathrm{~V}$, onde observamos uma adsorção que segue o modelo de ponte enquanto que sobre o cluster $\mathrm{Pt}_{5} \mathrm{a}$ adsorção segue o modelo de ponte sem dissociação da ligação O—O. Por outro 
lado, a adsorção sobre $\mathrm{Pt}_{4} \mathrm{Cr}$ segue o modelo de Pauling, com o oxigênio molecular adsorvido em apenas um sítio do cluster, sem a quebra da ligação $\mathrm{O}-\mathrm{O}$. Curvas de superfície de energia potencial mostraram um valor de aproximadamente 0,62 eV para a barreira de dissociação da ligação $\mathrm{O}-\mathrm{O}$ adsorvido sobre o cluster $\mathrm{Pt}_{4} \mathrm{Al}$ e aproximadamente $0,20 \mathrm{eV}$ para a barreira energética de dissociação da ligação $\mathrm{O}-$ $\mathrm{O}$ adsorvido sobre o cluster $\mathrm{Pt}_{4} \mathrm{~V}$.

Palavras-Chave: Adsorção de oxigênio, clusters de platina, Teoria do Funcional da Densidade. 


\begin{abstract}
This work presents a study applying the Density Functional Theory (DFT) using the B3LYP functional to study the electronic structure of platinum nanoclusters doped with $\mathrm{Al}, \mathrm{Cr}$ and $\mathrm{V}$ and their interactions with molecular oxygen. The analysis of Mulliken populations and NBO for the interaction between $\mathrm{O}_{2}$ and $\mathrm{Pt}_{2}, \mathrm{Pt}-\mathrm{Cr}$ and $\mathrm{Pt}-\mathrm{V}$ showed that charge transfer occurs orbital sed metals for the $p$ orbitals of oxygen, resulting in the filling of the antibonding orbitals of the molecule oxygen, causing the cleavage of $\mathrm{O}-\mathrm{O}$ and bond forming hybridized Metal - $\mathrm{O}$, bond dissociation energy of $\mathrm{O}-\mathrm{O}$ on Pt2 about $1.0 \mathrm{eV}$. On Pt-Cr and Pt-V, this value decreases to $0.56 \mathrm{eV}$ and $0.20 \mathrm{eV}$, respectively. Studies of the interaction between molecular oxygen and the $\mathrm{Pt} 3$ clusters, $\mathrm{Pt}_{2} \mathrm{Al}, \mathrm{Pt}_{2} \mathrm{Cr}$ and $\mathrm{Pt}_{2} \mathrm{~V}$ show that no dissociative adsorption occurs for molecular oxygen on the cluster $\mathrm{Pt}_{3}$ where we observe a second adsorption model bridge while on clusters $\mathrm{Pt}_{2} \mathrm{Al}$ and $\mathrm{Pt}_{2} \mathrm{~V}$ dissociative adsorption occurs of molecular oxygen. Moreover, with the cluster $\mathrm{Pt}_{2} \mathrm{Cr}$ adsorption follows the model of Pauling, with molecular oxygen adsorbed on only one site in the cluster, which was the $\mathrm{Pt}$ atom, without the cleavage of the O-O. Curves of potential energy surface for dissociation of molecular oxygen on $\mathrm{Pt}_{2} \mathrm{Al}$ and $\mathrm{Pt}_{2} \mathrm{~V}$ showed a value of about $0.21 \mathrm{eV}$ for the bond dissociation barrier of the $\mathrm{O}-\mathrm{O}$ adsorbed on the cluster $\mathrm{Pt}_{2} \mathrm{Al}$ approximately $0.30 \mathrm{eV}$ and for the barrier The bond dissociation O-O adsorbed on the cluster $\mathrm{Pt}_{2} \mathrm{~V}$. Studies of the interaction between molecular oxygen and clusters $\mathrm{Pt}_{4}, \mathrm{Pt}_{3} \mathrm{Al}, \mathrm{Pt}_{3} \mathrm{Cr}$ and $\mathrm{Pt}_{3} \mathrm{~V}$ show that no dissociative adsorption occurs for molecular oxygen on clusters $\mathrm{Pt}_{4}$ and $\mathrm{Pt}_{3} \mathrm{Cr}$ where it was observed that the adsorption follows the model of the bridge. On the other hand, on clusters $\mathrm{Pt}_{3} \mathrm{Al}$ and $\mathrm{Pt}_{3} \mathrm{~V}$ adsorption of molecular oxygen also followed the model bridge, with bond dissociation $\mathrm{O}-\mathrm{O}$. We found a value of $0.46 \mathrm{eV}$ for the barrier to bond dissociation to O-O adsorbed on the cluster $\mathrm{Pt}_{3} \mathrm{Al}$ and approximately $0.28 \mathrm{eV}$ for bond dissociation of the O-O adsorbed on the cluster $\mathrm{Pt}_{3} \mathrm{~V}$. Studies on the interaction between molecular oxygen and clusters $\mathrm{Pt}_{5}, \mathrm{Pt}_{4} \mathrm{Al}, \mathrm{Pt}_{4} \mathrm{Cr}$ and $\mathrm{Pt}_{4} \mathrm{~V}$ revealed that occurs dissociative adsorption of molecular oxygen on clusters $\mathrm{Pt}_{4} \mathrm{Al}$ and $\mathrm{Pt}_{4} \mathrm{~V}$, where we observe an adsorption model that follows the bridge while on the adsorption cluster $\mathrm{Pt}_{5}$ follows the bridge model without the bond dissociation. Moreover, adsorption is modeled on $\mathrm{Pt}_{4} \mathrm{Cr}$ Pauling with oxygen adsorbed on only one site of the cluster, without the cleavage of the O-O. Curves of potential energy surface showed a value of about $0.62 \mathrm{eV}$ for the bond
\end{abstract}


dissociation barrier of the $\mathrm{O}-\mathrm{O}$ adsorbed on the cluster $\mathrm{Pt}_{4} \mathrm{Al}$ and approximately 0.20 $\mathrm{eV}$ energy barrier for bond dissociation of the $\mathrm{O}-\mathrm{O}$ adsorbed on the $\mathrm{Pt}_{4} \mathrm{~V}$ cluster.

Keywords: Oxygen adsorption, platinum clusters and Density Functional Theory. 


\section{INTRODUÇÃO}

\subsection{A Reação de Redução de Oxigênio}

As reações eletroquímicas envolvendo o oxigênio, em particular a reação de redução de oxigênio (RRO), continuam a despertar o interesse dos eletroquímicos por envolverem complexidades cinéticas, pela necessidade de melhores eletrocatalisadores e pela importância destas reações em sistemas de conversão de energia eletroquímica, como as células a combustível, baterias metal-ar e eletrolisadores, além de sínteses químicas, processos biológicos e de corrosão. Assim, nas últimas décadas, particularmente motivados pelo desenvolvimento da tecnologia de células a combustível, muitos esforços têm sido dedicados ao estudo da reação de redução de oxigênio (RRO) em soluções aquosas ácidas e alcalinas sobre diferentes materiais eletródicos [1-7]. Entre as principais metas permanecem o aumento da atividade eletrocatalítica dos materiais de eletrodos existentes e o desenvolvimento de novos eletrocatalisadores.

A RRO é uma reação multieletrônica que inclui várias etapas elementares (cinética completa) no mecanismo reacional. Tanto em eletrólitos ácidos como em alcalinos, a RRO ocorre segundo dois mecanismos globais já conhecidos $[8,9]$ :

\section{i) Mecanismo de redução direta ou mecanismo 4 elétron}

Soluções Alcalinas:
$\mathrm{O}_{2}+2 \mathrm{H}_{2} \mathrm{O}+4 \mathrm{e}^{-} \rightarrow 4 \mathrm{OH}^{-}$
$E^{\circ}=+0,401 V$

Soluções Ácidas: 
$\mathrm{O}_{2}+4 \mathrm{H}^{+}+4 \mathrm{e}^{-} \rightarrow 2 \mathrm{H}_{2} \mathrm{O}$

$E^{\circ}=+1,229 V$

ii) Mecanismo com formação de peróxido

Soluções Alcalinas:

$\mathrm{O}_{2}+\mathrm{H}_{2} \mathrm{O}+2 \mathrm{e}^{-} \rightarrow \mathrm{HO}_{2}^{-}+\mathrm{OH}^{-}$

$E^{\circ}=-0,065 V$

seguido por redução de peróxido

$\mathrm{HO}_{2}^{-}+\mathrm{H}_{2} \mathrm{O}+2 \mathrm{e}^{-} \rightarrow 3 \mathrm{OH}^{-}$

$E^{\circ}=-0,867 V$

ou decomposição química

$2 \mathrm{HO}_{2}^{-} \rightarrow 2 \mathrm{OH}^{-}+\mathrm{O}_{2}$

Soluções Ácidas:

$\mathrm{O}_{2}+2 \mathrm{H}^{+}+2 \mathrm{e}^{-} \rightarrow \mathrm{H}_{2} \mathrm{O}_{2}$

$E^{\circ}=+0,670 V$

sendo que o peróxido de hidrogênio pode sofrer decomposição química:

$2 \mathrm{H}_{2} \mathrm{O}_{2} \rightarrow 2 \mathrm{H}_{2} \mathrm{O}+\mathrm{O}_{2}$

ou o peróxido de hidrogênio pode ser reduzido numa etapa posterior:

$\mathrm{H}_{2} \mathrm{O}_{2}+2 \mathrm{H}^{+}+2 \mathrm{e}^{-} \rightarrow 2 \mathrm{H}_{2} \mathrm{O} \quad \mathrm{E}^{\circ}=+1,770 \mathrm{~V}$

A distinção entre os dois mecanismos é dificultada, pois a redução direta também pode envolver a formação de peróxido de hidrogênio, sendo que este não sofre dessorção ou decomposição, e sua redução ocorrendo posteriormente. Em potenciais mais catódicos, a redução direta a água ocorre paralelamente à formação de peróxido. A técnica de eletrodo de disco-anel rotatório (RRDE - Rotating ring-disk electrode) é a mais utilizada para verificar e diferenciar os dois mecanismos, sendo que o eletrodo 
anel é utilizado somente como um sensor para monitoramento de produção de peróxido produzido no eletrodo de disco [5,7-9].

As possíveis rotas reacionais e o número de elétrons envolvidos na RRO estão relacionadas com os diferentes tipos de adsorção da molécula de $\mathrm{O}_{2}$. Na Figura 1 são apresentadas as prováveis formas de adsorção da molécula de $\mathrm{O}_{2}$. De acordo com a Figura 1, existem três modelos pelas quais as moléculas de oxigênio podem se adsorver na superfície do eletrodo, sendo então conhecidas como modelos de Griffith, Pauling e Ponte (também conhecido como modelo Yeager) [9-11]:<smiles>[X]C(=O)O</smiles><smiles>[M]1oo1</smiles>

I

Modelo de Griffith

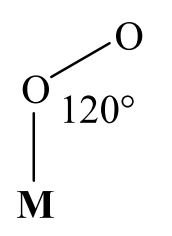

II<smiles>[M]O[M]O[M]</smiles>

III

\section{Modelo de Pauling}

Modelo de Ponte

Figura 1: Modelos de adsorção de oxigênio na superfície do eletrodo.

Os modelos de Griffith e Ponte favorecem a redução de oxigênio diretamente à água (mecanismo $4 \mathrm{e}^{-}$), enquanto que o modelo de Pauling favorece o mecanismo $2 \mathrm{e}^{-}$, resultando como produto $\circ \mathrm{H}_{2} \mathrm{O}_{2}$ notando-se que neste caso não ocorre a quebra da ligação O-O. Adicionalmente, as formas de adsorção poderão ocorrer simultaneamente e a preponderância de um ou outro mecanismo dependerá dos impedimentos estéricos, das propriedades eletrônicas e do espaçamento entre os sítios ativos, ou em outras palavras, do material eletródico e/ou das condições experimentais empregadas $[10,11]$. Sobre a platina, em meio ácido, a reação ocorre em várias etapas, e a primeira, onde ocorre a adsorção do $\mathrm{O}_{2}$ na superfície: 
$\mathrm{O}_{2}+\mathrm{Pt} \rightarrow \mathrm{Pt}-\mathrm{O}_{2}$

e a segunda, uma etapa eletroquímica dada por:

$\mathrm{Pt}-\mathrm{O}_{2}+\mathrm{H}^{+}+\mathrm{e}^{-} \rightarrow$ Produtos

que corresponde à etapa determinante da velocidade da reação. Do ponto de vista cinético, as demais etapas são poucos importantes, pois ocorrem após a etapa determinante da velocidade da reação.

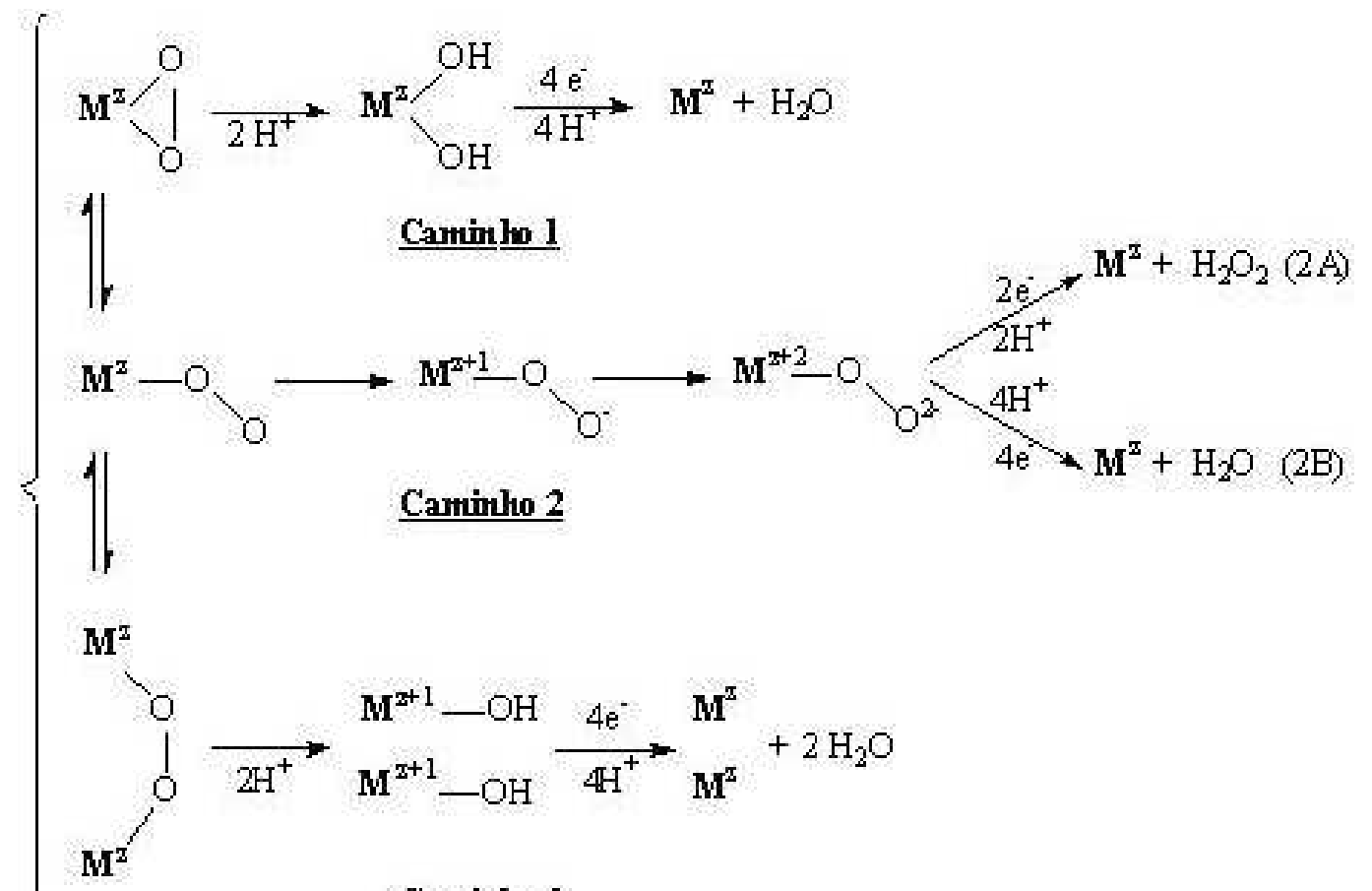

\section{Caminho 3}

Figura 2: Possíveis rotas reacionais para a RRO em soluções ácidas.

Em eletrólitos ácidos, platina e ligas de platina são considerados os melhores catalisadores para a redução de oxigênio, tanto em termos de menores sobrepotenciais desejados quanto em relação à estabilidade requerida em condições operacionais de 
uma célula a combustível. Sobre platina e metais da família da platina ocorre o mecanismo paralelo, mas a predominância é do mecanismo de redução direta a água (4 elétrons). Existem duas propostas para a primeira etapa reacional sobre estes metais. A primeira foi feita por Damjanovic et al. [12], que propõe uma transferência de prótons ocorrendo simultaneamente com uma transferência de carga, envolvendo $\mathrm{O}_{2} \leftrightarrow$ $\mathrm{O}_{2(\mathrm{ads})}$ ' seguido pela etapa determinante da velocidade da reação que é a transferência de carga $\mathrm{O}_{2(\mathrm{ads})}+\mathrm{H}^{+}+\mathrm{e}^{-} \rightarrow \mathrm{O}_{2} \mathrm{H}_{(\mathrm{ads})}$. A segunda proposta é de Yeager [9], cuja redução direta à água envolve primeiramente adsorção dissociativa da molécula de oxigênio sobre a superfície de platina que, provavelmente, ocorre simultaneamente com a transferência de carga, conforme ilustra o esquema da Figura 2.

Nas últimas décadas, estudos voltados para melhorar a atividade catalítica e a estabilidade de catalisadores para a RRO têm demonstrado a possibilidade de aplicação de ligas de platina (Pt-Cr, Pt-V, Pt-Fe, Pt-Ni, Pt-Co, Pt-Co-Cr, Pt-Ti, Pt-Al, etc.) em células a combustível [13-20]. Assim, catalisadores baseados em ligas de vários metais de transição são empregados para aumentar a atividade catalítica e reduzir custos. Com isso, Jalan e Taylor [13] estudaram diversas ligas de platina suportadas em carbono em células de ácido fosfórico (PAFC) e propuseram que a melhora na atividade catalítica resulta da diminuição da distância interatômica Pt-Pt nas ligas em comparação com a platina pura. A mesma interpretação foi apresentada por Apleby [14] e por Mukerjee e Srinivasan [15]. Entretanto, Glass et al. [16] propuseram que a melhora é específica para ligas altamente dispersas, porque esse efeito não foi encontrado para aglomerados de ligas Pt-Cr. Paffet et al. [17] realizaram um estudo da RRO sobre Pt-Cr em PAFC e mostraram um aumento na área superficial depois de um 
prolongado tempo de operação, devido à dissolução do metal não nobre. Beard e Ross [18] atribuem o aumento na atividade catalítica à presença das bordas de partículas com planos vicinais sobre a superfície dos catalisadores dispersos. Toda et al. [19] propuseram um mecanismo novo para a reação RRO sobre ligas Pt-M, baseado num aumento de vacâncias na banda 5-d da Pt, causada pela ligação metálica. Baseado nesse modelo, o aumento das vacâncias facilita a doação dos elétrons $2 \pi$ do oxigênio aos átomos de platina da superfície, facilitando a adsorção de $\mathrm{O}_{2}$ além de enfraquecer a ligação de O-O, aumentando a atividade eletrocatalítica. Recentemente, utilizando técnicas de espectroscopia de absorção de raios-X (XAS) Mukerjee et al. [20] mostraram que a melhora na atividade eletrocatalítica da platina deve-se a efeitos eletrônicos (vacâncias na banda $5 \mathrm{~d}$ da Pt), geométricos (distância Pt-Pt favorável e o número de coordenação da $\mathrm{Pt}$ ) e seu efeito sobre a quimissorção de espécies $\mathrm{OH}$ do eletrólito. Este estudo mostrou uma melhora na atividade catalítica na ordem $\mathrm{PtCr} / \mathrm{C}>$ $\mathrm{PtFe} / \mathrm{C}>\mathrm{PtMn} / \mathrm{C}>\mathrm{PtCo} / \mathrm{C}>\mathrm{PtNi} / \mathrm{C}>\mathrm{Pt}$. Aricó et al. [21] realizaram estudos de difração de raios-X para $\mathrm{Pt}$, $\mathrm{Pt}-\mathrm{Co}$ e $\mathrm{Pt}-\mathrm{Co}-\mathrm{Cr}$ e os resultados mostraram que $\mathrm{Pt}$ suportada em carbono tem estrutura cúbica de face centrada (fcc), porém, Pt-Co e PtCo-Cr suportados em carbono têm estrutura de face centrada tetragonal, o que está de acordo com a proposta de Glass [16]. Stamenkovic et al. [22] realizaram um estudo sobre a atividade de ligas $\mathrm{Pt}_{3} \mathrm{Ni}$ e $\mathrm{Pt}_{3} \mathrm{Co}$ com composições bem definidas e compararam com a Pt policristalina em soluções $\mathrm{H}_{2} \mathrm{SO}_{4} \quad 0,5 \mathrm{~mol} \mathrm{dm}^{-3}$ e $\mathrm{HClO}_{4} 0,1 \mathrm{~mol} \mathrm{dm}^{-3}$, num intervalo de temperatura entre 293 e $333 \mathrm{~K}$ e concluíram que a ordem da atividade eletrocatalítica depende da natureza dos ânions do eletrólito suporte. $\mathrm{Em}_{2} \mathrm{SO}_{4}$ : a atividade decresce na ordem $\mathrm{Pt}_{3} \mathrm{Ni}>\mathrm{Pt}_{3} \mathrm{Co}>\mathrm{Pt}$; e em $\mathrm{HClO}_{4}$ : "Pt-skin" $>\mathrm{Pt}_{3} \mathrm{Co}>\mathrm{Pt}_{3} \mathrm{Ni}$ 
$>$ Pt. Utilizando a técnica de RRDE foi encontrado que os parâmetros cinéticos da RRO e a produção de peróxido sobre as ligas é a mesma que Pt. O aumento na atividade catalítica para as ligas foi atribuído à inibição da formação de $\mathrm{Pt}-\mathrm{OH}_{\mathrm{ads}}$ sobre sítios de Pt.

\section{2- A Teoria do Funcional da Densidade (DFT)}

O objetivo principal da química quântica é obter soluções da equação de Schrödinger para determinar de forma precisa as propriedades de sistemas atômicos e moleculares. A equação de Schrödinger é uma equação de autovalores: quando um operador atua sobre uma função e resulta na mesma função multiplicada por uma constante. A função de onda $\boldsymbol{\Psi}$ é a autofunção do operador hamiltoniano $\widehat{\boldsymbol{H}}$, correspondente ao autovalor E (energia total do sistema). O operador Hamiltoniano pode ser escrito como:

$$
\widehat{H}=-\sum_{i=1}^{N} \frac{1}{2} \nabla_{i}^{2}-\sum_{A=1}^{M} \frac{1}{2 M_{A}} \nabla_{A}^{2}-\sum_{i=1}^{N} \sum_{A=1}^{M} \frac{Z_{A}}{\left|r_{i}-R_{A}\right|}+\sum_{i=1}^{N} \sum_{j>i}^{N} \frac{1}{\left|r_{i}-r_{j}\right|}+\sum_{A=1}^{M} \sum_{B>A}^{M} \frac{Z_{A} \cdot Z_{B}}{\left|R_{A}-R_{B}\right|}
$$

onde temos, respectivamente:

1) Energia cinética dos elétrons

2) Energia cinética do núcleo

3) Interação coulombica entre o núcleo e os elétrons

4) Interação coulombica entre os elétrons

5) Interação coulombica entre os núcleos 
A descrição da função de onda exata implica em uma descrição completa do sistema, ou seja, é possível calcular qualquer propriedade do mesmo, já que se têm todas as informações necessárias para as predições das propriedades atômicas.

Na prática, a equação de Schrödinger só possui solução exata para o átomo de hidrogênio ou cátions hidrogenóides. Para átomos polieletrônicos ou moléculas é necessário fazermos aproximações. A principal aproximação feita no sentido de resolver a equação de Schrödinger consiste na aproximação de Born e Oppenheimer [23], que permite a separação dos movimentos nucleares e eletrônicos, simplificando, assim, o tratamento do sistema. Essa aproximação considera o fato que, sendo os núcleos mais pesados que os elétrons, os núcleos movem-se lentamente em relação ao movimento dos elétrons. Assim, uma boa aproximação é obtida quando consideramos os elétrons na molécula movendo-se em um campo fixo formado pelos núcleos. Desse modo, o termo correspondente a energia cinética dos núcleos pode ser desprezado e a energia potencial de repulsão núcleo-núcleo pode ser considerada constante [23]. Logo, considerando a aproximação de Born-Oppenheimer e desprezando quaisquer efeitos relativísticos, o problema consiste em resolver a equação de Schrödinger, desacoplando o movimento eletrônico do movimento nuclear, reduzindo assim, o problema de $\mathrm{N}$ partículas a $\mathrm{N}$ problemas de partículas independentes:

$$
\widehat{H}(\vec{r}, \vec{R})=-\sum_{i=1}^{N} \frac{1}{2} \nabla_{\mathrm{i}}^{2}-\sum_{i=1}^{N} \sum_{A=1}^{M} \frac{Z_{A}}{\left|r_{i}-R_{A}\right|}+\sum_{i=1}^{N} \sum_{j>i}^{N} \frac{1}{\left|r_{i}-r_{j}\right|}
$$


onde temos, respectivamente:

1) Energia cinética dos elétrons

2) Interação coulombica entre o núcleo e os elétrons

3) Interação coulombica entre os elétrons

Entretanto, essa solução só é possível e exata para sistemas pequenos de átomos e moléculas. Para sistemas grandes, como sólidos, a solução torna-se fora de alcance. Existe, no entanto, outra maneira de resolver o problema, em que o objeto fundamental é a densidade eletrônica [ $\rho(\mathrm{r})$ ]. Ou seja, a equação de Schrödinger de N elétrons com a função de onda com $3 \mathrm{~N}$ variáveis (se não considerarmos o spin) pode ser escrita como uma equação da densidade eletrônica com somente três variáveis. A solução exata dessa equação foi dada por Hohenberg e Kohn (HK), em 1964, e é conhecida como "Teoria do Funcional da Densidade" (DFT - Density Functional Theory).

A teoria do funcional da densidade (DFT) tornou-se, nas útlimas décadas, um importante método para o estudo de estrutura eletônica de sólidos e moléculas. Problemas que antes eram tratados por métodos Hartree-Fock e pós Hartree-Fock são, agora, tratados utilizando-se a DFT, o que possibilita, em alguns casos, resultados que apresentam melhores concordâncias com os resultados experimentais disponíveis. A grande vantagem da metodologia do funcional da densidade sobre os métodos ab initio padrões está no ganho em velocidade computacional e espaço em memória [24]. Em contraste com métodos semi-empíricos, o hamiltoniano na DFT é bem definido e suas características não são obscurecidas por freqüentes aproximações no procedimento 
computacional. Nas derivadas das equações do DFT, nenhum parâmetro em princípio necessita ser ajustado ou determinado empiricamente.

As primeiras aplicações da densidade eletrônica [ $\rho(r)$ ] como meio de obtenção de energia de sistemas com mais de um elétron inicia-se quando Drude aplicou a teoria dos gases a um metal, considerado como gás homogêneo de elétrons, para desenvolver a sua teoria sobre condução térmica e elétrica [25]. Desde então, vários modelos (Drude, Sommerfeld, Thomas, Fermi, Dirac) foram propostos e aperfeiçoados. Thomas e Fermi $[26,27]$ propuseram um novo esquema baseado na densidade eletrônica dos sistemas, [ $\rho(r)$ ]. Com isso, torna-se mais simples a solução da equação de Schrödinger para sistemas grandes, uma vez que a complicada função de ondas de $\mathrm{N}$ elétrons na equação de Schrödinger é substituída pela densidade eletrônica, que é muito mais simples. Assim, utilizando-se de argumentos estatísticos para aproximar a distribuição de um gás de elétrons e desenvolver o funcional de energia, esse modelo assume que as propriedades eletrônicas dos sistemas reais sejam similares àquelas dos gases de férmions, isso é, um ensemble clássico de partículas não interagentes. Em seguida, o modelo de Thomas-Fermi foi aperfeiçoado para incluir, em adição, a energia de troca para um gás de elétrons desenvolvido por Dirac [24]. O funcional de energia [ $E(\rho)$ ] de Thomas-Fermi-Dirac é escrito como:

$$
E(\rho)=\lambda \int \rho^{\frac{5}{3}} d^{3} r-e \int \rho v_{N} d^{3} r-\frac{1}{2} e \int \rho v_{e} d^{3} r+U_{N N}-\frac{3}{4} e^{2}\left(\frac{3}{\pi}\right)^{\frac{1}{3}} \int \rho^{\frac{4}{3}} d^{3} r
$$

Impondo que $\mathrm{E}(\rho)$ seja estacionário com respeito a variações na densidade eletrônica $\rho$, teremos a equação de Thomas-Fermi-Dirac (TFD) [28]. 


$$
\rho=\frac{8 \pi}{3 h^{3}}\left(2 m e^{3}\right)^{\frac{3}{2}}\left[\frac{\left(2 m e^{3}\right)^{\frac{1}{2}}}{h}+\left(v-v(R)+\frac{\left(2 m e^{3}\right)}{h}\right)^{\frac{1}{2}}\right]^{3}
$$

A equação de Thomas-Fermi-Dirac (TFD) pode ser resolvida apenas numericamente e a solução da equação é independente do número de partículas. Porém, estes modelos de funcionais clássicos (TF e TFD) não podem competir em precisão com métodos auto-consistentes como, por exemplo, o método Hartree-Fock. O modelo de TFD falha ao reproduzir a estrutura quântica de camadas dos átomos. Além disso, o método não prediz ligações moleculares e os resultados para átomos não são muito precisos [28]. Apesar do limitado sucesso em reproduzir as propriedades dos sistemas reais, o modelo de Thomas-Fermi foi o protótipo da teoria do funcional da densidade, que focaliza a densidade eletrônica como variável fundamental.

A teoria do funcional da densidade está alicerçada em dois teoremas propostos por Hohenberg e Kohn [29] que podem ser definidos, independente da forma como o método do funcional está sendo apresentado, como:

1. A função de onda do estado fundamental e daí todas as propriedades deste estado são funcionais da densidade eletrônica $[\rho(r)]$;

2. A energia do estado fundamental de um sistema multieletrônico sob um dado potencial externo $v(r)$, pode ser escrito como:

$$
E[\rho(r)]=\int v(r) \rho(r) d^{3} r+F[\rho]
$$


Onde $F[\rho]$ é denominado funcional universal de $\rho(r)$, que independe do potencial externo $v(r)$. E substituindo a função densidade eletrônica exata deste estado por outra função aproximada $\tilde{\rho}(r)$, de modo que $\tilde{\rho}(r) \geq 0$ e

$$
\int \rho(r) d r=N
$$

a energia total será sempre maior ou igual a energia exata do sistema, ou seja:

$$
\tilde{E}=\tilde{E}[\tilde{\rho}(r)]=\int v(r) \tilde{\rho}(r) d^{3} r+F[\tilde{\rho}] \geq E[\rho(r)]=E_{0}
$$

pode-se, então, definir um funcional universal

$$
F[\rho]=\langle\Psi|\widehat{T}+\widehat{U}| \Psi\rangle
$$

pois $\mathrm{T}$ e $\mathrm{U}$ aplicam-se universalmente a todos os sistemas coulombianos. Entretanto, motivado pelo fato que as interações de Coulomb são de longo alcance, é conveniente separar do funcional universal $F[\rho]$ a parte coulombiana clássica, Kohn e Sham [30] apresentaram uma estratégia para calcular a estrutura eletrônica de sistemas de muitas partículas em que a energia do estado fundamental de um gás de elétrons não homogêneo interagindo com um potencial externo estático $v(r)$ é escrito como: 


$$
E[\rho(r)]=\int v(r) \rho(r) d^{3} r+\frac{1}{2} \iint \frac{\rho(r) \rho\left(r^{\prime}\right)}{\left|r-r^{\prime}\right|} d^{3} r d^{3} r^{\prime}+G[\rho]
$$

onde $G[\rho]$ também é um funcional universal, podendo ser escrito na forma

$$
G[\rho]=T_{0}[\rho]+E_{x c}[\rho]
$$

sendo $T_{0}[\rho]$ a energia cinética dos elétrons não interagentes e $E_{x c}[\rho]$ contém a energia de troca e a energia de correlação de um sistemas interagente com densidade $\rho(r)$. Sendo assim, o funcional de energia do estado de um sistema multieletrônico pode ser escrito

$$
E[\rho(r)]=\int v(r) \rho(r) d^{3} r+\frac{1}{2} \iint \frac{\rho(r) \rho\left(r^{\prime}\right)}{\left|r-r^{\prime}\right|} d^{3} r d^{3} r^{\prime}+T_{0}[\rho]+\int \rho(r) E_{x c}[\rho] d^{3} r
$$

De acordo com o teorema variacional, tomando a variação de $E[\rho(r)]$, com o vínculo que a carga eletrônica total seja fixa e aplicando a condição de extremo incluindo o vínculo obtemos

$$
\int \delta \rho(r)\left\{\frac{\delta T_{0}}{\delta \rho}+v(r)+\int \frac{\rho\left(r^{\prime}\right)}{\left|r-r^{\prime}\right|} d^{3} r^{\prime}+v_{x c}[\rho]-\mu\right\} d^{3} r=0
$$

que é a equação de Euler-Lagrange para um sistemas de partículas não interagentes movendo-se sob um potencial efetivo, sendo $v_{x c}[\rho]$ o potencial de troca-correlação

$$
v_{x c}[\rho]=\frac{\delta E_{x c}}{\delta \rho}
$$


e a energia cinética dos elétrons não interagentes pode ser escrito como:

$$
T_{0}[\rho]=-\frac{1}{2} \sum_{i} \int \psi_{i}^{*} \nabla^{2} \psi_{i} d^{3} r
$$

A solução da equação acima pode ser obtida resolvendo a equação de Schrödinger com Hamiltoniano monoeletrônico para um sistema auxiliar de partículas não interagentes

$$
\hat{h}^{K S} \psi_{i}(r)=\left(-\frac{1}{2} \nabla^{2}+v^{K S}[\rho]\right) \psi_{i}(r)=\epsilon_{i} \psi_{i}(r)
$$

com

$$
\rho(r)=\sum_{i=1}^{N}\left|\psi_{i}(r)\right|^{2}
$$

onde $\boldsymbol{v}^{\boldsymbol{K}}[\boldsymbol{\rho}]$ é o potencial efetivo de Kohn-Sham dado por

$$
v^{K S}[\rho]=v(r)+\int \frac{\rho\left(r^{\prime}\right)}{\left|r-r^{\prime}\right|} d^{3} r^{\prime}+v_{x c}[\rho]
$$

Dessa forma, $\boldsymbol{v}^{K S}$ depende de $\rho$ e, portanto, as 3 equações cima devem ser resolvidas autoconsistentemente e são conhecidas como equações Kohn-Sham [31]. Na Figura 3 é mostrado um esquema representativo do ciclo de autoconsistencia, onde, o processo interativo começa com uma aproximação para a densidade $\rho(r)$, em 
seguida obtêm-se $\boldsymbol{v}^{\boldsymbol{K} \boldsymbol{S}}(\boldsymbol{r})$ e encontra-se uma nova densidade $\rho^{\prime}(r)$ aplicando a equação de Schrödinger, até que $\rho(r)=\rho^{\prime}(r)$ para uma dada tolerância previamente estabelecida, sendo necessário fazer uma escolha a priori do funcional de trocacorrelação, $E_{x c}[\rho]$.

Assim, estabeleceu-se o caminho mais popular que leva ao formalismo de Hohenberg e Kohn a sua aplicação prática e o sucesso do método Kohn - Sham devese ao fato de não estar inteiramente baseado em funcionais da densidade, incorporando ao seu ciclo autoconsistente de equações, o conceito de orbitais monoeletrônicos.

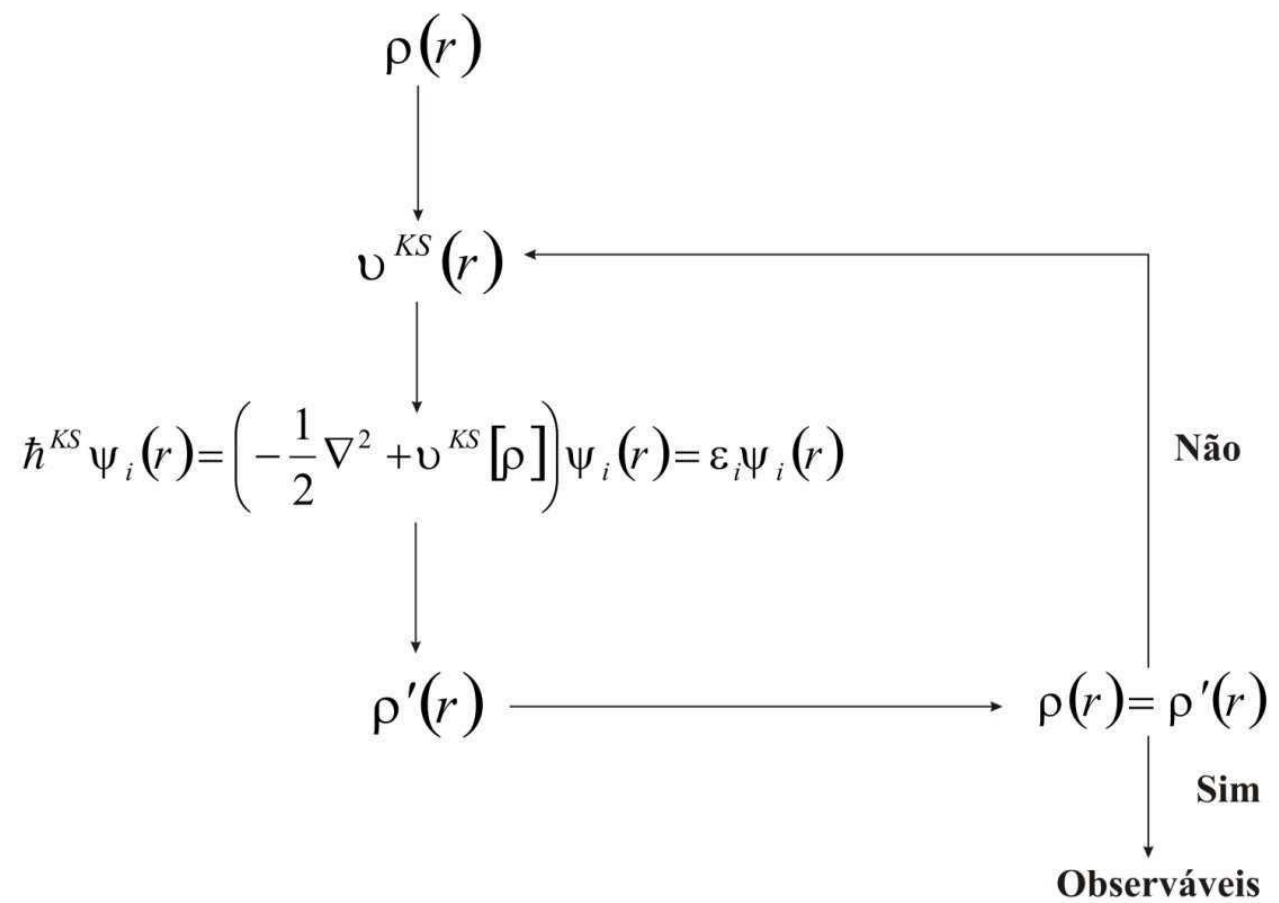

Figura 3: Esquema representativo do ciclo de autoconsistência. 
Como conseqüência, a DFT praticada nas equações de Kohn e Sham assemelha-se a uma teoria monoeletrônica, embora todos os efeitos de muitos corpos ainda sejam incluídos por meio de um único termo, o funcional de troca-correlação, onde aplicação prática do DFT depende inteiramente de uma aproximação simples e suficientemente precisa para $E_{x c}[\rho]$.

Apesar das equações de Kohn e Sham incorporarem a energia cinética $T[\rho]$, elas ainda deixam o termo de troca-correlação indeterminado. É necessário, portanto, encontrar uma maneira de contornar esta dificuldade. A mais simples aproximação para $\boldsymbol{T}[\boldsymbol{\rho}]$ foi apresentada por Kohn e Sham em 1965 e é conhecida como Aproximação Local da Densidade (LDA- Local Density Approximation) para a energia de trocacorrelação. Na aproximação LDA a energia de troca correlação para um sistema de elétrons homogêneo de densidade $\rho(r)$ no ponto $r$ é assumida como sendo igual à energia de troca correlação de um gás de elétrons homogêneo com a mesma densidade. Desse modo, tanto a energia de troca quanto a energia de correlação do gás homogêneo de elétrons são usadas localmente para aproximar $E$ do sistema sob estudo.

$$
E_{x c}^{L D A}[\rho]=\int \rho(r) \epsilon_{x c}^{h}[\rho(r)] d^{3} r
$$

onde $\epsilon_{x c}^{h}[\rho]$ é a energia de troca correlação por elétron de um gás de elétrons homogêneo de densidade $\rho=\rho(r)$. Sendo assim, tem-se:

$$
v_{x c}[\rho] \equiv \frac{d}{d \rho}\left\{\rho(r) \epsilon_{x c}^{h}[\rho(r)]\right\}
$$


Como conseqüência, a qualidade da descrição do sistema inomogêneo interagente depende de quão inomogêneo é sua densidade. Logo, seria de se esperar que a LDA fornecesse bons resultados apenas quando aplicada a sistemas nos quais a densidade varia pouco com relação à posição. Entretanto, em parte devido ao cancelamento sistemático de erros que tipicamente subestima a parte de correlação e superestima a parte de troca, a LDA tem mostrado sucesso quando aplicada no estudo de sistemas bem diferentes do líquido de elétrons tais como átomos, moléculas, sólidos e clusters [32]. Entretanto, sistemas como estes possuem densidades inomogêneas, e apesar dos resultados razoáveis, a precisão química ainda não foi alcançada [33]. Com isso, uma maneira de melhorar os resultados obtidos com LDA seria incluir gradientes ou suas derivadas com relação às coordenadas espaciais, na dependência do funcional. Portanto, a taxa de variação da densidade de sistemas interagentes poderia ser melhor descrita. A primeira tentativa foi expandir a densidade em gradientes como em uma série de potências, onde é desenvolvida uma série de funcionais chamados Gradient-Expansion Approximations (GEA) [34]. Posteriormente, percebeu-se que isso poderia ser feito de forma mais geral e sistemática, gerando, dessa maneira, a família dos funcionais conhecidos como Generalized-Gradient Approximations (GGA) [34, 35].

$$
E_{x c}^{G G A}[\rho]=\int f[\rho(r), \nabla \rho(r)] d^{3} r
$$

Existem várias propostas para o functional $E_{x c}^{G G A}[\rho]$. Na física são usadas propriedades conhecidas do funcional desenvolvido por Perdew, Burke e Ernzerhof (PBE), em 1996 [36]. Na química, por outro lado, é comum o ajuste de parâmetros 
usando conjuntos selecionados de moléculas, como é o caso do funcional B3LYP, no qual a parte de troca é a aproximação estabelecida por Becke [37], em 1988, e a parte de correlação foi dada por Lee, Yang e Parr [38], no mesmo ano.

Os funcionais de correlação devem combinar-se com os funcionais de troca, sendo estes largamente utilizados. O funcional híbrido B3LYP (ou Becke3LYP - o número 3 indica que este funcional possui três parâmetros), que é definido por:

$$
E_{x c}^{B 3 L Y P}=\left(1-a_{0}-a_{x}\right) E_{X}^{L S D A}+a_{0} E_{X}^{H F}+a_{x} E_{X}^{B 88}+\left(1-a_{c}\right) E_{C}^{V W N}+a_{c} E_{C}^{L Y P}
$$

Onde o termo $E_{X}^{L S D A}$ denota o funcional de troca baseado no método do gás uniforme de elétrons, $E_{X}^{H F}$ é o termo de troca nas equações Hartree-Fock, o termo $E_{X}^{B 88}$ é o funcional de Becke de $1988, \boldsymbol{E}_{C}^{V W N}$ denota o funcional de correlação desenvolvido por Vosko-Wilk-Nusair no procedimento LSDA e $E_{C}^{L Y P}$ utiliza o funcional de correlação de LYP. Os valores das constantes obtidas pelos ajustes com valores experimentais das energias de atomizações moleculares são: $a_{0}=0,20 ; a_{x}=0,72$ e $a_{c}=0,81$. [23]. O B3LYP tem sido o funcional de maior uso em cálculos de química quântica nos últimos anos devido à sua parametrização; portanto, ele foi o funcional escolhido para realização do estudo teórico abordado nesta tese. 


\section{3- Estudos Teóricos da Reação de Redução de Oxigênio}

Estudos que aplicaram a Teoria do Funcional da Densidade (DFT) com o funcional GGA-PW91 para investigar a adsorção de oxigênio atômico sobre diferentes metais de transição foram realizados por Hammer e NØrskov [40], com o intuito de entender as propriedades que afetam a energia de adsorção. Para adsorção sobre a superfície $\mathrm{Pt}(111)$, os cáculos reproduziram a estrutura observada experimentalmente e o calor de adsorção foi 2,68 eV. Esse resultado está em boa concordância com o valor 2,4 eV obtido experimentalmente para um quarto de uma monocamada de oxigênio adsorvido. De acordo com esses autores, três propriedades superficiais contribuem para melhora na facilidade da quebra de ligação dos adsorbatos: i) A energia do centro da banda-d, ii) o grau de ocupação da banda-d (número de elétrons d) e iii) o acoplamente entre a densidade dos estados do elemento e a densidade dos estados do metal de acordo com a abaixo:

$$
E_{d-h y b}=-2(1-f) \frac{V^{2}}{\left|\varepsilon_{d}-\varepsilon_{a}\right|}+2(1+f) \alpha V^{2}
$$

Estudos computacionais da adsorção de oxigênio sobre $\operatorname{Pt}(111)$ foi reportado por Eichler e Hafner [41]. Eles também aplicaram a Teoria do Funcional da Densidade (DFT) com o funcional GGA-PW91 e identificaram dois precursores distintos, porém quase degenerados, adsorvidos quimicamente sobre $\operatorname{Pt}(111)$ com distâncias de 1,8 $1,9 \AA$ A $O$ primeiro precursor foi o superóxido formado com a molécula adsorvida em ponte com comprimento de ligação de 1,39 Å, freqüência de estiramento da ligação 0 - 
$\mathrm{O}$ de $850 \mathrm{~cm}^{-1}$ e energia de adsorção de $0,72 \mathrm{eV}$. O segundo precursor foi o peróxido ligado entre três átomos de platina da superfície, com energia de adsorção de 0,68 eV, comprimento da ligação $\mathrm{O}-\mathrm{O}$ de $1,43 \AA$ com frequência de estiramento de $710 \mathrm{~cm}^{-1}$. Hyman and Medlin [42] estudaram o efeito do campo elétrico nas energias de adsorção de oxigênio atômico e molecular e, também, sobre a energia de dissociação de oxigênio molecular sobre $\mathrm{Pt}(111)$. Os autores verificaram que campos elétricos positivos, que correspondem a superfícies carregadas positivamente provocam uma diminuição na energia de adsorção das espécies oxigenadas enquanto isso, campos elétricos negativos aumentam a energia de adsorção. Os autores mostraram que essas variações dependem fortemente da quantidade de átomos $\mathrm{Pt}$ no cluster $\mathrm{Pt}(111)$. Na superfície com 10 átomos de platina, a energia de adsorção de oxigênio atômico decresce cerca de $0,25 \mathrm{eV}$ na presença de um campo elétrico de 0,51 V/Å. Os Resultados obtidos para oxigênio molecular adsorvido e o seu estado de transição mostraram comportamentos similares.

Feibelman et al. [43] estimaram a energia de adsorção dos átomos de oxigênio sobre a superfície $\mathrm{Pt}(111)$. Esses autores observaram que o número de coordenação da platina foi um fator importante na seletividade do sitio de adsorção e encontraram que sítios fcc são preferenciais em relação aos sítios hcp por cerca de $0,4 \mathrm{eV}$. Xu et al. [44] realizaram estudos semelhantes para a adsorção de oxigênio atômico e molecular, além da dissociação de $\mathrm{O}_{2}$ sobre ligas $\mathrm{Pt}_{3} \mathrm{Co}$ e $\mathrm{Pt}_{3} \mathrm{Fe}$, e sobre uma monocamada de $\mathrm{Pt}$ sobre essas duas ligas. Eles mostraram uma relação linear entre a energia de ligação e a barreira de dissociação de $\mathrm{O}_{2}$ sobre os metais de transição e as ligas estudadas. Os resultados de DFT mostraram que átomos de Co na superfície de $\mathrm{Pt}_{3} \mathrm{Co}$ permitiram a adsorção dissociativa de $\mathrm{O}_{2}$ mais facilmente que sobre a superfície Pt (111). A energia 
de ativação sobre $\mathrm{Pt}_{3}$ Co foi $0,24 \mathrm{eV} / \mathrm{O}_{2}$ comparado com $0,77 \mathrm{eV} / \mathrm{O}_{2}$ sobre $\mathrm{Pt}$ pura, e a energia de ligação foi maior para $\mathrm{Pt}_{3} \mathrm{Co}\left(0,92\right.$ eV/O $\mathrm{O}_{2}$ e 4,29 eV/O) em comparação com Pt pura $\left(0,62 \mathrm{eV} / \mathrm{O}_{2}\right.$ e $\left.3,88 \mathrm{eV} / \mathrm{O}\right)$. Eles observaram que a monocamada de $\mathrm{Pt}$ sobre $\mathrm{Pt}_{3} \mathrm{Co}(111)$ foi a superfície menos reativa em termos de energia de ligação $\left(0,34 \mathrm{eV} / \mathrm{O}_{2}\right.$ e 3,50 eV/O) e resultados similares foram encontrados para $\mathrm{Pt}_{3} \mathrm{Fe}(111)$.

Balbuena et al. [45] realizaram estudos da redução de oxigênio em ligas de Co, Ni e Cr, embutidos em matrizes de Pt, usando o método DFT com o funcional B3PW91 combinado com o pseudopotencial LANL2DZ. Os autores postularam que $\mathrm{Cr}$ e Co podem atuar como sítios ativos para a dissociação de $\mathrm{O}_{2}$ em vez de serem oxidados como sítios de sacrifício e identificaram XPt e XXPt ( $\mathrm{X}=\mathrm{Cr}$ e Co) como sítios mais ativos para promover a dissociação de $\mathrm{O}_{2}$. Gobal et al. [46] estudaram a adsorção de oxigênio atômico e molecular sobre nano clusters $\mathrm{Pd}_{\mathrm{x}} \mathrm{Cu}_{3-\mathrm{x}}(\mathrm{x}=0-3)$ neutros e carregados negativamente usando a metodologia B3PW91/LANL2TZ(f). Foi observado que as formas e as energias de adsorção dependem fortemente da carga e da composição dos clusters. O Modo de Adsorção mais estável para oxigênio molecular sobre todos os clusters neutros foi o modelo ponte, com oxigênio adsorvido sobre os sítios Pd-Cu, com energia de adsorção $-103,7 \mathrm{~kJ} \mathrm{~mol}^{-1}$, enquanto que sobre os clusters carregados negativamente o modelo ponte sobre os sítios $\mathrm{Pd}-\mathrm{Pd}$, com energia de adsorção $-140,9 \mathrm{~kJ} \mathrm{~mol}^{-1}$ e sobre $\mathrm{Cu}-\mathrm{Cu}$, com energia de adsorção $-172,9 \mathrm{~kJ} \mathrm{~mol}^{-1}$, foram mais estáveis. Além disso, os autores mostraram que (i): o aumento do teor de cobre no cluster e (ii) a adição de carga negativa no cluster promove a quebra da ligação $\mathrm{O}-\mathrm{O}$, aumentando a atividade do cluster frente a dissociação de oxigênio molecular. 
Anderson e Albu realizaram estudos teóricos para a reação de redução de oxigênio [47]. Inicialmente eles estudaram o potencial reversível e a energia de ativação para a reação não catalisada de redução de oxigênio à água aplicando o método MP2/6-31G**. O valor calculado para o potencial reversível da reação foi de 1,18 V para a reação via $4 e^{-}$e $0,61 \mathrm{~V}$ para a reação ocorrendo via mecanismo $2 \mathrm{e}^{-}$.

$$
\begin{array}{llll}
\mathrm{O}_{2}(\mathrm{~g})+\mathrm{H}^{+}(\mathrm{aq})+\mathrm{e}^{-}(\mathrm{U}) & \rightarrow & \mathrm{HO}_{2} \cdot(\mathrm{aq}) \\
\mathrm{HO}_{2} \cdot(\mathrm{aq})+\mathrm{H}^{+}(\mathrm{aq})+\mathrm{e}-(\mathrm{U}) & \rightarrow & \mathrm{H}_{2} \mathrm{O}_{2}(\mathrm{aq}) \\
\mathrm{H}_{2} \mathrm{O}_{2}(\mathrm{aq})+\mathrm{H}^{+}(\mathrm{aq})+\mathrm{e}^{-}(\mathrm{U}) & \rightarrow & \mathrm{HO} \cdot(\mathrm{g}) \\
& & \mathrm{H}_{2} \mathrm{O}(\mathrm{aq}) \\
\mathrm{HO} \cdot(\mathrm{g}) & +\mathrm{H}^{+}(\mathrm{aq})+\mathrm{e}^{-}(\mathrm{U}) & \rightarrow & \mathrm{H}_{2} \mathrm{O}(\mathrm{aq})
\end{array}
$$

Estudos envolvendo macrociclos de metais de transição, particularmente, metaloftalocianinas e metaloporfirinas, para a redução de oxigênio em soluções aquosas têm sido realizados [48-50]. Esses compostos pertencem a uma classe que fornecem oportunidades singulares para se examinar em detalhes os fatores envolvidos na ativação e posterior redução da molécula de oxigênio. Tais fatores envolvem os efeitos associados com substituições nas posições axiais e periféricas do anel macrociclo, a natureza do centro metálico e o grau de conjugação do anel. Estudos da reatividade destes compostos com a molécula de oxigênio também são importantes para esclarecimentos de processos biológicos, de fotossíntese e de catálise. Adicionalmente, aspectos teóricos relacionados com este tema também despertam muita atenção, uma vez que permitem revelar o papel de efeitos localizado e coletivo, bem como estabelecer relações entre estrutura química e atividade catalítica. Zagal et al. [51-54] realizaram estudos aplicando os métodos PM3 (Parametric Method 3) e HF 
(Hartree-Fock) para compreender a reatividade de ftalocianinas de cobalto substituídas (CoPcs). Os autores calcularam parâmetros intermoleculares relacionados (doadorreceptor) e concluíram que:

$$
\eta_{D-R}=\frac{1}{2}\left(\varepsilon_{L U M O / R}-\varepsilon_{H O M O / D}\right)
$$

onde $\varepsilon_{\text {LUMO/R }}$ é a energia do LUMO (orbital molecular desocupado de menor energia) do receptor e $\varepsilon_{\text {HOMO/D }}$ é a energia do HOMO (orbital molecular ocupado de maior energia) do doador de elétrons. Quando a diferença de energia decresce, a interação entre os orbitais (doador e receptor) é mais forte e a reação torna-se mais rápida. 


\section{OBJETIVO}

O objetivo deste trabalho é estudar a interação entre oxigênio molecular e nanoclusters de platina $\left(\mathrm{Pt}_{\mathrm{n}}, \mathrm{n}=2-5\right)$ dopados com Alumínio, Cromo e Vanádio. Para este fim, será aplicado a Teoria do Funcional da Densidade (DFT), utilizando o funcional híbrido de três parâmetros de Becke com as correções de gradiente fornecidas pelos funcionais de Lee, Yang e Parr (B3LYP), para estudar a estrutura eletrônica destes clusters e a interação com oxigênio molecular. 


\section{DETALHES COMPUTACIONAIS}

Este estudo foi conduzido por meio de cálculos de estrutura eletrônica baseados na teoria do funcional da densidade. A otimização das geometrias das estruturas, além dos cálculos de energia de ligação, distribuição de cargas e freqüências vibracionais dos nano-clusters $\mathrm{Pt}-\mathrm{M}, \mathrm{Pt}_{2} \mathrm{M}, \mathrm{Pt}_{3} \mathrm{M}$ e $\mathrm{Pt}_{4} \mathrm{M}(\mathrm{M}=\mathrm{Cr}$, V e Al) e sua interação com oxigênio e intermediários da reação de redução de oxigênio, foram realizadas com a utilização do programa GAUSSIAN 03, Revision B04 [55]. Foram empregados cálculos baseados na teoria do funcional da densidade (DFT), utilizando o funcional híbrido de três parâmetros de Becke com as correções de gradiente fornecidas pelos funcionais de Lee, Yang e Parr (B3LYP) $[35,56,57]$. As funções de bases LANL2DZ (Los Alamos National Laboratory 2-double-z, usada para os átomos $\mathrm{Pt}, \mathrm{V}, \mathrm{Cr}, \mathrm{Al}, \mathrm{O}), 6-311 \mathrm{G}^{* *}$ (usada para o átomo O). A presença de polarização na camada d pode assegurar uma possível transferência de carga do substrato para o adsorbato. Os cálculos de frequências vibracionais das estruturas otimizadas foram usados para gerar as energias do ponto zero (ZPE), entalpias $(H)$, entropias (S), energias livres de Gibbs (G) e as correções do ponto zero.

A avaliação das estruturas eletrônicas foram realizadas através do método NBO (Natural Bond Orbitals), que localiza os orbitais canônicos e os transforma em orbitais de centro, orbitais de ligação, orbitais de caroço e de pares isolados, de acordo com a visão de estrutura química de Lewis. A análise dos Orbitais de NBO foi conduzida de maneira a encontrar as propriedades eletrônicas das estruturas mais estáveis e relevantes.

Na Figura 4 estão apresentadas as estruturas usadas como entrada (input) dos cálculos de otimização dos nano-clusters de platina, bem como os nano-clusters 
de platina dopados com com um átomo de alumínio, cromo e vanádio. Todas as possíveis multiplicidades de spin foram analisadas, no intuito de verificar a diferença energética entre as diferentes multiplicidades.
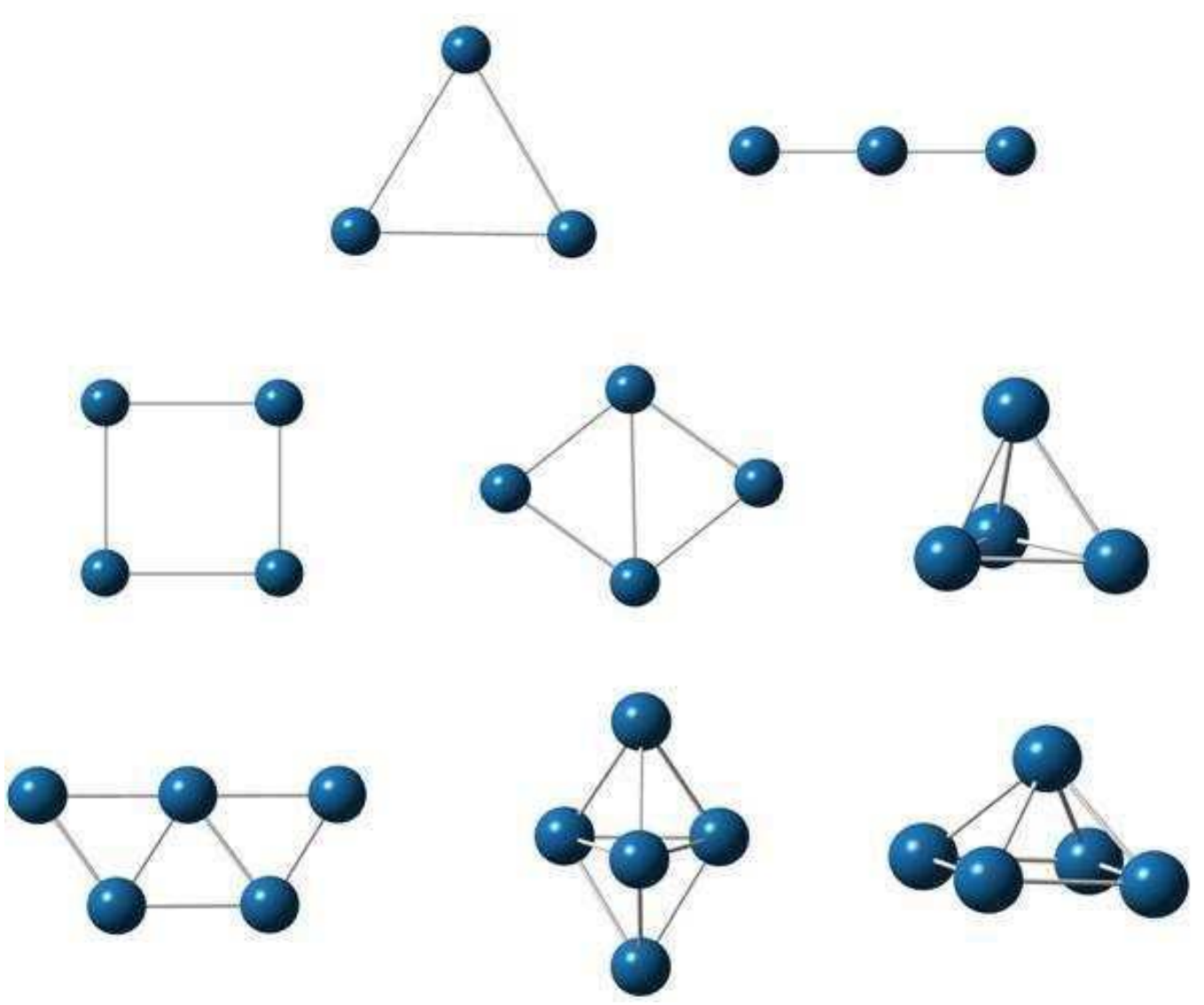

Figura 4: Estruturas usadas como entrada (input) dos cálculos de otimização dos nano-clusters $\mathrm{Pt}_{3}, \mathrm{Pt}_{4}$ e $\mathrm{Pt}_{5}$.

Na Figura 5 estão apresentadas as estruturas usadas como entrada (input) para os cálculos da interação entre oxigênio molecular e a estrutura $\mathrm{Pt}_{2} \mathrm{Cr}$. Cálculos semelhantes foram realizados para estudar a interação entre oxigênio molecular e todos clusters abordados nesta tese. Todas as possíveis multiplicidades de spin para estas estruturas foram analisadas, no intuito de encontrar a forma mais estável de adsorção. 


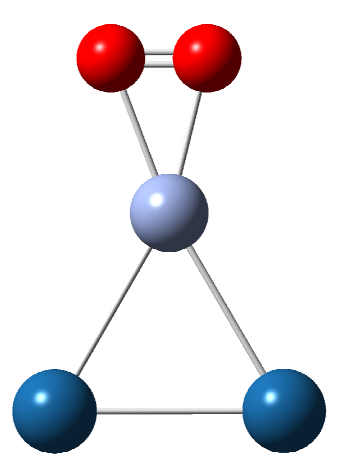

(a)

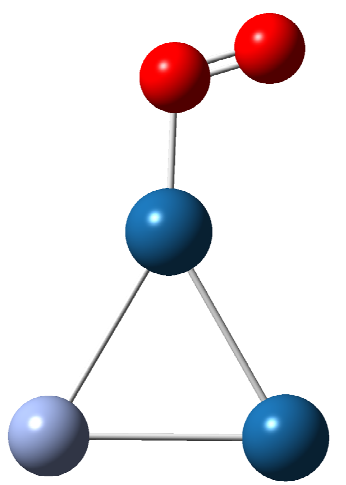

(b)

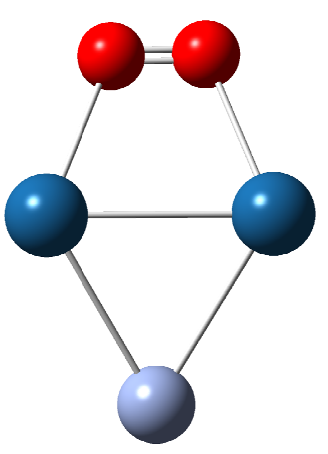

(c)

Figura 5: Formas de adsorção usadas como arquivos de entrada (input) para os cálculos da interação entre oxigênio molecular e a estrutura $\mathrm{Pt}_{2} \mathrm{Cr}$. (a) Modelo Griffith, (b) Modelo Pauling e (c) Modelo Ponte.

O valor da energia de ligação por átomo para os clusters foi calculado usando a seguinte equação:

$$
\mathbf{E}_{\mathbf{L}}(\text { Cluster })=\left[\mathbf{E}_{\text {total }}(\text { Cluster })-\mathbf{n}\left(\mathbf{E}_{\text {átomo }}\right)\right] / \mathbf{n}
$$

onde $E_{L}$ é a energia de ligação por átomo, $E_{\text {total }}$ a energia total do cluster e $n$ o número de átomos do elemento no cluster e $E_{\text {átomo }} a$ energia calculada para um átomo.

A energia de ligação entre oxigênio molecular e os clusters $\mathrm{Pt}-\mathrm{M}, \mathrm{Pt}_{2} \mathrm{M}, \mathrm{Pt}_{3} \mathrm{M}$ e $\mathrm{Pt}_{4} \mathrm{M}$ foi calculada segundo a equação:

$$
\mathbf{E}_{\mathrm{ads}}=\mathbf{E}_{\mathrm{Pt}-\mathrm{M}\left(\mathbf{O}_{2}\right)}-\mathbf{E}_{\mathrm{Pt}-\mathbf{M}}-\mathbf{E}_{\mathbf{O}_{2}}
$$




\section{RESULTADOS E DISCUSSÕES}

\subsection{Otimização e Propriedades Eletrônicas dos Clusters $\mathrm{Pt}_{2}, \mathrm{Pt}-\mathrm{M}$ e $\mathrm{M}_{2}(\mathrm{M}=\mathrm{Al}$, Cr e V)}

Na Tabela 1 são apresentados os resultados obtidos através dos cálculos usando o funcional B3LYP, combinado com a função de base LANL2DZ, para as distâncias interatômicas, energias totais, multiplicidades de spin, energias de ligação, potenciais verticais de ionização, afinidades eletrônicas e frequências vibracionais para os clusters diatômicos $\mathrm{Pt}_{2}, \mathrm{Al}_{2}, \mathrm{Pt}-\mathrm{Al}, \mathrm{Cr}_{2}, \mathrm{PtCr}, \mathrm{V}_{2}$ e $\mathrm{Pt}-\mathrm{V}$. Alguns resultados experimentais foram incluídos, entre parênteses, para fins de comparação. O estado fundamental para $\mathrm{Pt}_{2}$ é triplete, onde a ligação $\mathrm{Pt}-\mathrm{Pt}$ forma hibridização com os orbitais atômicos naturais, sendo $82 \%$ de contribuição 6 s e $18 \%$ de contribuição $5 \mathrm{~d}_{z}{ }^{2}$, com distância interatômica igual a 2,376 Å e frequência vibracional de $284,82 \mathrm{~cm}^{-1}$. Gupta et al. [58] encontraram, através de estudos de STM (Scanning tunneling microscope), um valor médio de $2,45 \AA$ para o comprimento de ligação do dímero $\mathrm{Pt}_{2}$ adsorvido sobre grafite. Balbuena et al. [59] realizaram cálculos teóricos usando a metodologia B3PW91/LANL2DZ e obtiveram o valor para distância interatômica do dímero $\mathrm{Pt}_{2}$ igual a 2,41 $\AA$. O valor encontrado para a energia de ligação para o cluster $\mathrm{Pt}_{2}$ foi 1,31 eV/átomo, resultado em concordância com os obtidos experimentalmente por Taylor et al. [60] através da técnica R2PI (Resonant two-photon ionization spectroscopy) que foi de 1,37 eV/átomo. 
Tabela 1: Geometrias otimizadas, propriedades energéticas e multiplicidade de spin para os clusters $\mathrm{Pt}_{2}, \mathrm{Al}_{2}, \mathrm{Cr}_{2}, \mathrm{~V}_{2}, \mathrm{Pt}-\mathrm{Al}, \mathrm{Pt}-\mathrm{Cr}$ e Pt-V. Método B3LYP/LANL2DZ.

\begin{tabular}{|c|c|c|c|c|c|c|c|}
\hline Cluster & m & $d_{M-M}(A)$ & $\mathrm{E}_{0}$ (hartree) & $E_{L}(e V)$ & E.A. $(\mathrm{eV})$ & E.I. $(\mathrm{eV})$ & $v\left(\mathrm{~cm}^{-1}\right)$ \\
\hline Pt-Pt & 3 & $2,376(2,45)^{61}$ & $-238,252$ & $-1,31$ & $1,98(1,89)^{62}$ & $9,28(8,70)^{61}$ & 284,82 \\
\hline Al-Al & 3 & 2,854 & $-3,910$ & $-0,65$ & $1,18(1,15)^{63}$ & $5,86(5,40)^{64}$ & 235,14 \\
\hline Pt-Al & 2 & 2,290 & $-121,140$ & $-1,75$ & 1,82 & 7,80 & 364,45 \\
\hline $\mathrm{Cr}-\mathrm{Cr}$ & 5 & 1,800 & $-172,441$ & $-1,27$ & $0,39(0,50)^{65}$ & $6,75(6,40)^{66}$ & 598,94 \\
\hline $\mathrm{Pt}-\mathrm{Cr}$ & 5 & 2,365 & $-205,426$ & $-1,10$ & 1,42 & 7,69 & 213,97 \\
\hline$V-V$ & 3 & 1,754 & $-142,256$ & $-0,30$ & $0,40(0,26)^{67}$ & $6,35(6,35)^{68}$ & 706,80 \\
\hline Pt-V & 4 & 2,266 & $-190,426$ & $-1,56$ & 1,31 & 7,52 & 310,13 \\
\hline
\end{tabular}

O estado fundamental encontrado para $\mathrm{Al}_{2}$ é triplete com distância interatômica igual a 2,854 A e frequência vibracional $235,14 \mathrm{~cm}^{-1}$. Os resultados de afinidade eletrônica e potencial vertical de ionização estão próximos com resultados obtidos experimentalmente obtidos por Gantefoer et. al. [63]. O cluster $\mathrm{Cr}_{2}$ com multiplicidade de spin igual a cinco foi o estado de menor energia enquanto que para o $V_{2}$ o estado fundamental é o estado triplete e os resultados de energia de ionização para o cluster $V_{2}$ estão em boa concordância com resultados obtidos experimentalmente por James et al. [68] usando a técnica R2PI. Para os clusters bimetálicos Pt-Al, Pt-Cr e Pt-V o estado fundamental encontrado foi duplete, quinteto e quarteto, respectivamente. Podemos observar na Tabela 2 que os átomos de metais não nobres $\mathrm{Al}, \mathrm{Cr}$ e $\mathrm{V}$ cedem carga para os átomos de platina e as análises de NBO mostram que existe transferência de carga dos orbitais s dos átomos $\mathrm{Cr}$ e V para orbitais d da platina e transferência de carga dos orbitais s e p do Al para os orbitais d da platina. A ligação entre Pt-Al é formada com hibridização de orbitais, sendo $29 \%$ de $\operatorname{Al}\left[3 s(7 \%)+3 p_{z}(93 \%)\right]$ e $71 \%$ de Pt[6s $\left.(86 \%)+5 d_{z}^{2}(14 \%)\right]$. Esta é uma ligação de caráter $\sigma_{s-p}$ com contribuição majoritária da Platina para o spin $\alpha .0$ Alumínio tem participação maior no spin $\beta$, formando uma ligação $\sigma_{s-s} \operatorname{com} 88 \%$ de 
Al[3s $(99,9 \%)]$ com 12\% de Pt[6s(97\%)]. No cluster Pt-Cr a ligação $\mathrm{Pt}-\mathrm{Cr}$ é formada basicamente devido a contribuição dos orbitais $\mathrm{s}$ e d de cada átomo, com duas ligações no spin a: a natureza da primeira ligação é de $56 \%$ de $\operatorname{Pt}\left[6 s(94 \%)+5 d_{z} 2\right.$ $(5 \%)]$ e $44 \% \operatorname{Cr}\left[4 s(93 \%)+3 d_{x} 2 y 2(7 \%)\right]$ e na segunda, ligação $\sigma_{d-d}$,temos $28 \%$ de $\operatorname{Pt}\left[5 d_{z} 2(99 \%)\right]$ e $72 \%$ de $\operatorname{Cr}\left[3 d_{x} 2-y 2(99,9 \%)\right]$. Além disso, foi observado uma ligação $\sigma_{s-s}$ no spin $\beta$ com $75 \%$ de $\operatorname{Pt}\left[6 s(96 \%)+5 d_{z} 2(4 \%)\right]$ e $25 \%$ de $\operatorname{Cr}\left[4 s(89 \%)+3 d_{x} 2-y 2\right.$ (10\%)]. Para o cluster Pt-V a ligação $\mathrm{Pt}-\mathrm{V}$ também é formada devido a contribuição dos orbitais s e d de cada átomo, envolvendo duas ligações no spin $\alpha$ : a natureza da primeira ligação é $\sigma_{s}$ - s com $52 \%$ de $\operatorname{Pt}[6 s(98 \%)]$ e $48 \%$ V[4s(99\%)] e na segunda, ligação $\sigma_{d-d}$,temos $73 \%$ de $\operatorname{Pt}\left[5 d_{x} 2-y 2(100 \%)\right]$ e $27 \%$ de $V\left[3 d_{x} 2-y 2(98 \%)\right]$. Por fim, também foi observado uma ligação no spin $\beta$ com $66 \%$ de $\operatorname{Pt}\left[6 s(95 \%)+5 d_{x} 2-y 2\right.$ $(5 \%)]$ e $34 \%$ de $V\left[4 s(89 \%)+3 d_{z} 2(11 \%)\right]$

Tabela 2: Cargas atômicas de Mulliken e configurações eletrônicas dos átomos presentes nos clusters heteronucleares Pt-Al, Pt-Cr e Pt-V.

\begin{tabular}{|c|c|c|c|}
\hline Cluster (Pt-M) & $\mathbf{q}_{\mathrm{Pt}}(\mathbf{e})$ & $\mathbf{q}_{\mathrm{M}}(\mathbf{e})$ & Configuração eletrônica (NBO) \\
\hline $\mathrm{Pt}-\mathrm{Al}$ & $-0,577$ & $+0,577$ & $\mathrm{Pt}=[$ core $] 6 \mathrm{~S}^{0.89} 5 \mathrm{~d}^{9.63} 6 \mathrm{p}^{0.01}$ \\
& & & $\mathrm{Al}=\left[\right.$ core] $3 \mathrm{~S}^{1.83} 3 \mathrm{p}^{0.63}$ \\
\hline $\mathrm{Pt}-\mathrm{Cr}$ & $-0,539$ & $+0,539$ & $\begin{array}{c}\mathrm{Pt}=[\text { core }] 6 \mathrm{~S}^{1.33} 5 \mathrm{~d}^{9.14} 6 \mathrm{p}^{0.01} \\
\mathrm{Cr}=[\text { core }] 4 \mathrm{~S}^{0.72} 3 \mathrm{~d}^{4.78} 4 \mathrm{p}^{0.02}\end{array}$ \\
\hline $\mathrm{Pt}-\mathrm{V}$ & $-0,548$ & $+0,548$ & $\begin{array}{c}\mathrm{Pt}=[\text { core }] 6 \mathrm{~S}^{1,11} 5 \mathrm{~d}^{9.38} 6 \mathrm{p}^{0.01} 6 \mathrm{~d}^{0.01} \\
\mathrm{~V}=[\text { core }] 4 \mathrm{~S}^{0.81} 3 \mathrm{~d}^{3.59} 4 \mathrm{p}^{0.04}\end{array}$ \\
\hline
\end{tabular}




\subsection{Otimização e Propriedades Eletrônicas dos Clusters $\mathrm{Pt}_{3}$ e $\mathrm{Pt}_{2} \mathrm{M}$ (M=Al, $\mathrm{Cr}$ e V)}

$\mathrm{Na}$ Figura 5 estão apresentadas as geometrias encontradas para as estruturas de menor energia dos clusters $\mathrm{Pt}_{3}, \mathrm{Pt}_{2} \mathrm{Al}, \mathrm{Pt}_{2} \mathrm{Cr}$ e $\mathrm{Pt}_{2} \mathrm{~V}$ otimizadas com o método B3LYP/LANL2DZ e na Tabela 3, estão reunidas informações sobre distâncias interatômicas, multiplicidades de spin, configurações eletrônicas dos átomos, potenciais verticais de ionização e afinidades eletrônicas. Podemos observar na Tabela 3 que os átomos de metais não nobres $\mathrm{Al}$, $\mathrm{Cr}$ e $\mathrm{V}$ cedem carga para os átomos de platina e as análises de NBO mostram que existe transferência de carga dos orbitais $\mathrm{s}$ dos átomos $\mathrm{Cr}$ e $\mathrm{V}$ para orbitais $\mathrm{d}$ da platina e transferência de carga dos orbitais $s$ e $p$ do Al para os orbitais $d$ da platina. A estrutura mais estável do cluster $\mathrm{Pt}_{3}$ é singlete com geometria triangular (equilátero), energia total 357,4275 hartree, potencial vertical de ionização 7,98 eV e afinidade eletrônica 2,17 eV. Ervin et al. [62] estudaram a estrutura eletrônica e vibracional de trímeros de metais de transição e encontraram o valor 1,87 eV para a afinidade eletrônica do trímero $\mathrm{Pt}_{3}$. De acordo com a análise dos orbitais naturais observamos que as três ligações formadas nesse cluster são idênticas, com contribuições perfeitamente simétricas de ambos os átomos de $\mathrm{Pt}$, hibridizados também da mesma maneira $\left(\mathrm{s}^{48 \%}\right.$ $+\mathrm{d}^{51 \%}$ ). O estado fundamental encontrado para $\mathrm{Pt}_{2} \mathrm{Al}$ é duplete com geometria triangular (isósceles), energia total -240,300 hartree, potencial vertical de ionização 7,21 eV e afinidade eletrônica 2,58 eV. As análises de NBO mostraram que no spin alfa temos uma ligação sigma entre $\mathrm{Pt1}-\mathrm{Pt2}$ onde ambos os átomos de $\mathrm{Pt}$ contribuem com $50 \%$ nas ligações com simetria e hibridização igual de ambos os átomos $\left(s^{87 \%}+d^{11 \%}\right)$, formando uma ligação $\sigma_{s-s}$ entre esses átomos. No spin $\beta$ ocorrem mais duas ligações simétricas eletronicamente, entretanto uma é do tipo d-d 
envolvendo orbitais $d$ em praticamente $100 \%$ e a outra é hibridizada $\left(s^{85 \%}+d^{14 \%}\right)$, formando, assim como no spin alfa, uma ligação simples s-s.

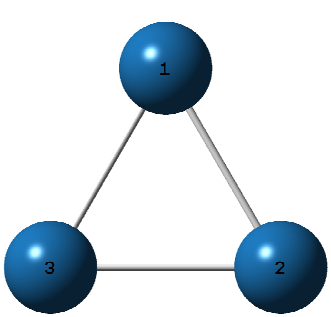

(a)

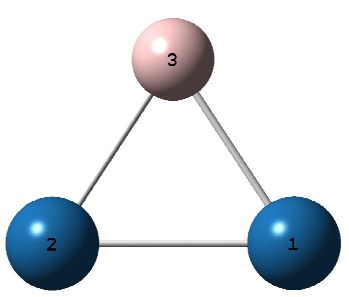

(b)

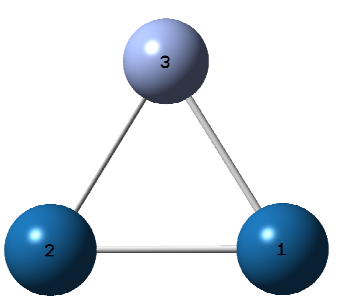

(c)

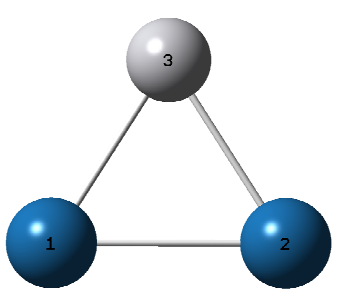

(d)

Figura 5: Geometrias das estruturas (a) $\mathrm{Pt}_{3}$, (b) $\mathrm{Pt}_{2} \mathrm{Al}$, (c) $\mathrm{Pt}_{2} \mathrm{Cr}$ e (d) $\mathrm{Pt}_{2} \mathrm{~V}$ otimizadas com o método B3LYP/LANL2DZ.

Tabela 3: Distâncias interatômicas, propriedades energéticas, multiplicidade de spin, cargas atômicas de Mulliken e configurações eletrônicas dos átomos presentes nos clusters $\mathrm{Pt}_{3}, \mathrm{Pt}_{2} \mathrm{Al}, \mathrm{Pt}_{2} \mathrm{Cr}$ e $\mathrm{Pt}_{2} \mathrm{~V}$.

\begin{tabular}{|c|c|c|c|c|c|c|}
\hline Cluster & m & $\mathbf{d}_{M-M}(\AA)$ & $\mathbf{Q}_{\mathrm{M}}(\mathbf{e})$ & Configuração eletrônica & E.A.(eV) & E.I.(eV) \\
\hline $\mathrm{Pt}_{3}$ & 1 & $\begin{array}{l}1-2=2.521 \\
1-3=2,521 \\
2-3=2,521\end{array}$ & $\begin{array}{l}\mathrm{Pt}_{1}=0,000 \\
\mathrm{Pt}_{2}=0,000 \\
\mathrm{Pt}_{3}=0,000\end{array}$ & $\begin{array}{l}\mathrm{Pt}_{1}\left[\text { core] } 6 S^{0.71} 5 d^{9.18} 6 p^{0.05}\right. \\
\mathrm{Pt}_{2} \text { [core]6S } 6 S^{0.77} d^{9.18} 6 p^{0.05} \\
\mathrm{Pt}_{3} \text { [core]6S } 6 S^{0.77} 5 d^{9.18} 6 p^{0.05}\end{array}$ & $2,17(1,87)^{62}$ & 7,98 \\
\hline $\mathrm{Pt}_{2} \mathrm{Al}$ & 2 & $\begin{array}{l}1-2=2.630 \\
1-3=2.400 \\
2-3=2,400\end{array}$ & $\begin{array}{l}\mathrm{Pt}_{1}=-0,426 \\
\mathrm{Pt}_{2}=-0,426 \\
\mathrm{Al}_{3}=+0,852\end{array}$ & $\begin{array}{l}\mathrm{Pt}_{1}\left[\text { core] } 6 \mathrm{~S}^{1.08} 5 \mathrm{~d}^{9.14} 6 \mathrm{p}^{0.10}\right. \\
\mathrm{Pt}_{2}\left[\text { core] } 6 \mathrm{~S}^{1.08} 5 \mathrm{~d}^{9.14} 6 \mathrm{p}^{0.10}\right. \\
\mathrm{Al}_{3}\left[\text { core] } 3 \mathrm{~S}^{1.43} 3 \mathrm{p}^{0.93} 4 \mathrm{p}^{0.01}\right.\end{array}$ & 2,58 & 7,21 \\
\hline $\mathrm{Pt}_{2} \mathrm{Cr}$ & 5 & $\begin{array}{l}1-2=2.565 \\
1-3=2.452 \\
2-3=2.452\end{array}$ & $\begin{array}{l}\mathrm{Pt}_{1}=-0,318 \\
\mathrm{Pt}_{2}=-0,318 \\
\mathrm{Cr}_{3}=+0,637\end{array}$ & $\begin{array}{l}\mathrm{Pt}_{1} \text { [core]6S } \mathrm{S}^{0.92} 5 \mathrm{~d}^{9.34} 6 \mathrm{p}^{0.05} \\
\mathrm{Pt}_{2} \text { [core]6S } 6 \mathrm{~S}^{0.92} 5 \mathrm{~d}^{9.34} 6 \mathrm{p}^{0.05} \\
\mathrm{Cr}_{3} \text { [core] } 4 \mathrm{~S}^{0.52} 3 \mathrm{~d}^{4.76} 4 \mathrm{p}^{0.10}\end{array}$ & 1,35 & 7,25 \\
\hline $\mathrm{Pt}_{2} \mathrm{~V}$ & 2 & $\begin{array}{l}1-2=2.986 \\
1-3=2.246 \\
2-3=2.246\end{array}$ & $\begin{array}{l}\mathrm{Pt}_{1}=-0,371 \\
\mathrm{Pt}_{2}=-0,371 \\
\mathrm{~V}_{3}=+0,742\end{array}$ & $\begin{array}{l}\text { Pt1[core]6S } S^{1.16} 5 d^{9.11} 6 p^{0.04} \\
\text { Pt2[core]6S } S^{1.16} 5 d^{9.11} 6 p^{0.04} \\
\text { V3[core]4S } S^{0.62} 3 d^{3.57} 4 p^{0.21} 4 d^{0.01}\end{array}$ & 1,65 & 6,78 \\
\hline
\end{tabular}

$\mathrm{O}$ estado fundamental encontrado para $\mathrm{Pt}_{2} \mathrm{Cr}$ é quinteto com geometria triangular (isósceles), energia total -324,616 hartree, potencial vertical de ionização $7,25 \mathrm{eV}$ e afinidade eletrônica $1,35 \mathrm{eV}$. No spin $\alpha$, a estrutura $\mathrm{Pt}_{2} \mathrm{Cr}$ forma uma ligação Pt - Pt com cada átomo de Pt doando 50\% de densidade e utilizado orbitais híbridos $[\mathrm{s}(46 \%)+\mathrm{d}(54 \%)]$. A ligação entre Pt1-Cr3 o átomo de Pt participa um pouco mais na sobreposição (59\%), mas ainda formando híbridos [Pt1 s(48\%) + 
$d(52 \%)$ ]. O mesmo foi observado para a ligação Pt2 - Cr3. Entretanto, No spin $\beta$ apenas a ligação $\mathrm{Pt}$ - Pt permanece simétrica em termos de densidade. Para as ligações $\mathrm{Pt}$ - Cr o átomo de platina forma ligações $\sigma$ hibridizadas com participação nas ligações de cerca de $83 \%$. Além disso, as análises revelaram que os átomos de cromo também sofrem hibridização $(s(48,6 \%)+d(51,4 \%)]$ nestas ligações. O estado fundamental encontrado para $\mathrm{Pt}_{2} \mathrm{~V}$ é duplete com geometria triangular (isósceles), energia total $-309,610$ hartree, potencial vertical de ionização 6,78 eV e afinidade eletrônica 1,65 eV. As análises de NBO destes clusters revelaram que no spin a temos múltiplas ligações para esta estrutura, onde as principais são: Pt1-V3, com 91\% de participação do átomo de $\mathrm{Pt}$ com seus orbitais d e $9 \%$ de $\mathrm{V}$ hibridizado $[p(50 \%)+d(50 \%)] ;$ Existem ainda outras duas ligações de dois centros entre estes átomos, só que com menor participação do átomo de Pt (87\%) e (64\%). Notamos nestas ligações que à medida que o átomo de $\mathrm{Pt}$ diminui sua participação na ligação, a participação dos orbitais d também diminuem. Temos também uma ligação Pt2-V3 com 91\% de participação do átomo de Pt com seus orbitais d e 9\% de $V$ hibridizado $[p(50 \%)+d(50 \%)]$. No spin $\beta$ existem duas ligações idênticas quanto à participação dos átomos e orbitais: em Pt1-V3 e Pt2-V3 a participação de ambos os átomos é de $70 \%$ de $\operatorname{Pt}[\mathbf{s}(80 \%)+\mathrm{d}(20 \%)]$ e $30 \%$ de $\mathrm{V}[\mathrm{s}(41 \%)+\mathrm{p}(12 \%)+$ $d(46 \%)]$.

Analisando a Tabela 3 observamos que o potencial vertical de ionização para $\mathrm{Pt}_{3}$ é 7,98 eV e quando um átomo de platina é substituído por $\mathrm{Al}, \mathrm{Cr}$ e $\mathrm{V}$ os valores dos potenciais verticais de ionização decrescem para 7,21 eV, 7,25 eV e 6,78 eV, respectivamente. Essa diminuição no potencial vertical de ionização favorece a transferência do cluster para o adsorbato, provocando uma alteração na reatividade para a adsorção e dissociação de $\mathrm{O}_{2}$ sobre estes clusters. 


\subsection{Otimização e Propriedades Eletrônicas dos Clusters $\mathrm{Pt}_{4}$ e $\mathrm{Pt}_{3} \mathrm{M}$ (M=Al, Cr e V)}

$\mathrm{Na}$ Figura 6 estão apresentadas as geometrias encontradas para as estruturas mais estáveis dos clusters $\mathrm{Pt}_{4}, \mathrm{Pt}_{3} \mathrm{Al}, \mathrm{Pt}_{3} \mathrm{Cr}$ e $\mathrm{Pt}_{3} \mathrm{~V}$ otimizadas com o método B3LYP/LANL2DZ e na Tabela 4, estão reunidas informações sobre distâncias interatômicas, multiplicidades de spin, configurações eletrônicas dos átomos, potenciais verticais de ionização e afinidades eletrônicas. Podemos observar na Tabela 4 que os átomos de metais não nobres $\mathrm{Al}$, $\mathrm{Cr}$ e V cedem carga para os átomos de platina e as análises de NBO mostram que existe transferência de carga dos orbitais $\mathrm{s}$ dos átomos $\mathrm{Cr}$ e $\mathrm{V}$ para orbitais $\mathrm{d}$ da platina e transferência de carga dos orbitais $s$ e $p$ do Al para os orbitais $d$ da platina. A estrutura mais estável do cluster $\mathrm{Pt}_{4}$ é triplete com geometria piramidal triangular, energia total 476,608 hartree, potencial vertical de ionização 6,67 eV e afinidade eletrônica 1,80 eV. De acordo com a análise dos orbitais naturais no cluster $\mathrm{Pt}_{4}$ temos apenas uma ligação no spin $\alpha$, formada pelos átomos Pt1-Pt2, completamente simétrica e hibridizada em $s^{84 \%}+p^{5 \%}+d^{11 \%}$ para ambos os átomos. No spin beta foram observadas múltiplas ligações: entre Pt1 e Pt3 temos uma ligação formada pela contribuição de $41 \% \operatorname{Pt} 1[\mathrm{~s}(46 \%)+\mathrm{d}(52 \%)]$ e $59 \%$ de Pt3 $[\mathrm{s}(32 \%)+\mathrm{d}(66 \%)]$; Por outro lado, a ligação formada entre os átomos Pt1 e Pt4, é resultante da ligação entre os orbitais hibridizados com contribuição de $42 \%$ de Pt1 $[s(47 \%)+d(53 \%)]$ e $58 \%$ de Pt4 $[s(33 \%)+d(67 \%)]$. As ligações entre os átomos Pt2-Pt3 e Pt2-Pt4 são idênticas às ligações encontradas para Pt1-Pt4. A última ligação observada no spin beta é formada entre os átomos Pt3-Pt4 com 50\% de overlap por parte de cada átomo e as seguintes hibridizações $s^{31 \%}+d^{67 \%}$, em que se nota mais uma vez que neste spin as ligações envolvendo os orbitais d são mais favorecidas. 


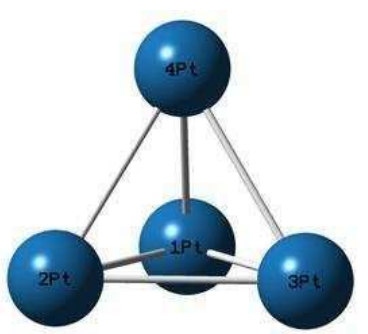

(a)

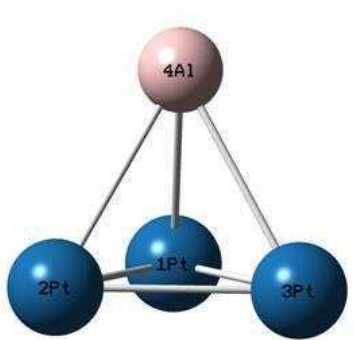

(b)

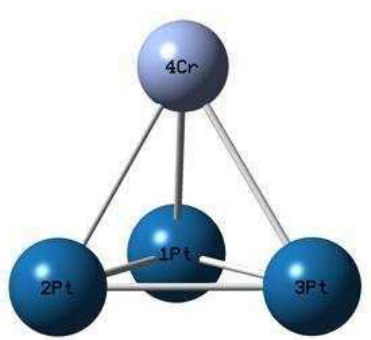

(c)

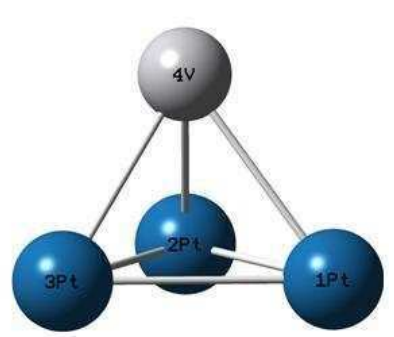

(d)

Figura 6: Geometrias das estruturas (a) $\mathrm{Pt}_{4}$, (b) $\mathrm{Pt}_{3} \mathrm{Al}$, (c) $\mathrm{Pt}_{3} \mathrm{Cr}$ e (d) $\mathrm{Pt}_{3} \mathrm{~V}$ otimizadas com o método B3LYP/LANL2DZ.

$\mathrm{O}$ estado fundamental encontrado para $\mathrm{Pt}_{3} \mathrm{Al}$ é duplete com geometria piramidal triangular, energia total $-359,50$ hartree, potencial vertical de ionização $7,06 \mathrm{eV}$ e afinidade eletrônica 2,19 eV. Para a estrutura otimizada $\mathrm{Pt}_{3} \mathrm{Al}$ temos apenas uma ligação no spin alfa, com simetria de overlap e maior participação dos orbitais s na ligação entre Pt2 e Pt3 $[s(90 \%)+d(8 \%)]$. No spin beta encontramos três ligações idênticas ocorrendo entre os átomos Pt1-Pt2, Pt1-Pt3 e Pt2-Pt3, onde observamos que as ligações ocorrem com $50 \%$ de contribuição de cada átomo com orbitais híbridos $[\mathrm{s}(44 \%)+\mathrm{d}(55 \%)]$ para todos os átomos.

Tabela 4: Distâncias interatômicas, propriedades energéticas, multiplicidade de spin, cargas atômicas de Mulliken e configurações eletrônicas dos átomos presentes nos clusters $\mathrm{Pt}_{4}, \mathrm{Pt}_{3} \mathrm{Al}, \mathrm{Pt}_{3} \mathrm{Cr}$ e $\mathrm{Pt}_{3} \mathrm{~V}$.

\begin{tabular}{|c|c|c|c|c|c|c|}
\hline Cluster & m & $\mathbf{d}_{M-M}(\AA)$ & $\mathbf{Q}_{M}(\mathbf{e})$ & Configuração eletrônica & E.A.(eV) & E.I.(eV) \\
\hline $\mathrm{Pt}_{4}$ & 3 & $\begin{array}{l}1-2=2,783 \\
1-3=2,636 \\
2-3=2,636 \\
1-4=2,636 \\
2-4=2,636 \\
3-4=2,623\end{array}$ & $\begin{array}{l}\mathrm{Pt}_{1}=+0,004 \\
\mathrm{Pt}_{2}=+0,004 \\
\mathrm{Pt}_{3}=-0,004 \\
\mathrm{Pt}_{4}=-0,004\end{array}$ & $\begin{array}{l}\mathrm{Pt}_{1}[\text { core }] 6 S^{0.65} 5 d^{9.29} 6 p^{0.09} \\
\mathrm{Pt}_{2}[\text { core }] 6 S^{0.65} 5 d^{9.29} 6 p^{0.09} \\
\mathrm{Pt}_{3}[\text { core }] 6 S^{0.65} 5 d^{9.21} 6 p^{0.11} \\
\mathrm{Pt}_{4}[\text { core }] 6 S^{0.65} 5 d^{9.21} 6 p^{0.11}\end{array}$ & 1,80 & 6,67 \\
\hline $\mathrm{Pt}_{3} \mathrm{Al}$ & 2 & $\begin{array}{l}1-2=2,587 \\
1-3=2,575 \\
2-3=2,569 \\
1-4=2,677 \\
2-4=2,679 \\
3-4=2,667\end{array}$ & $\begin{array}{l}\mathrm{Pt}_{1}=-0,231 \\
\mathrm{Pt}_{2}=-0,231 \\
\mathrm{Pt}_{3}=-0,231 \\
\mathrm{Al}_{4}=+0,694\end{array}$ & 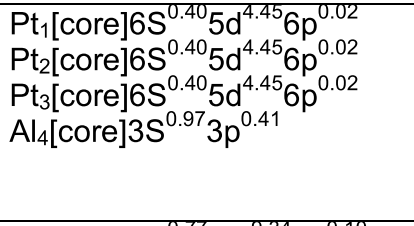 & 2,19 & 7,06 \\
\hline $\mathrm{Pt}_{3} \mathrm{Cr}$ & 7 & $\begin{array}{l}1-2=2,634 \\
1-3=2,634 \\
2-3=2,592 \\
1-4=2,664\end{array}$ & $\begin{array}{l}\mathrm{Pt}_{1}=-0,223 \\
\mathrm{Pt}_{2}=-0,223 \\
\mathrm{Pt}_{3}=-0,223 \\
\mathrm{Cr}_{4}=+0,669\end{array}$ & $\begin{array}{l}\mathrm{Pt}_{1}[\text { core }] 6 \mathrm{~S}^{0.71} 5 \mathrm{~d}^{9.34} 6 \mathrm{p}^{0.10} \\
\mathrm{Pt}_{2}\left[\text { core] } 6 \mathrm{~S}^{0.77} 5 \mathrm{~d}^{9.34} 6 \mathrm{p}^{0.10}\right. \\
\mathrm{Pt}_{3}\left[\text { core] } 6 \mathrm{~S}^{0.77} 5 \mathrm{~d}^{9.34} 6 \mathrm{p}^{0.10}\right. \\
\mathrm{Cr}_{4}\left[\text { core] } 4 \mathrm{~S}^{0.30} 3 \mathrm{~d}^{4.97} 4 \mathrm{p}^{0.13}\right.\end{array}$ & 1,31 & 6,61 \\
\hline
\end{tabular}




\begin{tabular}{|c|c|c|c|c|c|c|}
\hline & & $\begin{array}{l}2-4=2,664 \\
3-4=2,664\end{array}$ & & & & \\
\hline $\mathrm{Pt}_{3} \mathrm{~V}$ & 6 & $\begin{array}{l}1-2=2,609 \\
1-3=2,748 \\
2-3=2,748 \\
1-4=2,561 \\
2-4=2,561 \\
4-5=2,477\end{array}$ & $\begin{array}{l}\mathrm{Pt}_{1}=-0,235 \\
\mathrm{Pt}_{2}=-0,235 \\
\mathrm{Pt}_{3}=-0,305 \\
\mathrm{~V}_{4}=+0,776\end{array}$ & $\begin{array}{l}\mathrm{Pt}_{1}[\text { core }] 6 \mathrm{~S}^{0.80} 5 d^{9.25} 6 \mathrm{p}^{0.12} \\
\mathrm{Pt}_{2}[\text { core }] 6 \mathrm{~S}^{0.80} 5 \mathrm{~d}^{9.25} 6 \mathrm{p}^{0.12} \\
\mathrm{Pt}_{3}[\text { core }] 6 \mathrm{~S}^{1.08} 5 \mathrm{~d}^{9.13} 6 \mathrm{p}^{0.12} 6 \mathrm{~d}^{0.01} \\
\mathrm{~V}_{4}[\text { core }] 4 \mathrm{~S}^{0.38} 3 \mathrm{~d}^{3.71} 4 \mathrm{p}^{0.25} 4 \mathrm{~d}^{0.01}\end{array}$ & 1,64 & 7,30 \\
\hline
\end{tabular}

Para o cluster $\mathrm{Pt}_{3} \mathrm{Cr}$ o estado menos energético encontrado foi septeto com geometria piramidal triangular, energia total $-443,793$ hartree, potencial vertical de ionização 6,61 eV e afinidade eletrônica 1,31 eV. A ligações existentes neste cluster encontradas após análises de NBO foram uma ligação Pt no spin alfa entre Pt1-Pt2 com $50 \%$ de contribuição de cada átomo, formando uma ligação entre os orbitais híbridos Pt1[s(90\%) + p(10\%)] e Pt2 [s(90\%) + p(10\%)]. Para o spin beta as análises de NBO revelaram a existência de três ligações: a primeira entre Pt1-Pt2, formada pela contribuição de $50 \%$ de cada átomo resultante da ligação entre os orbitais híbridos $\operatorname{Pt} 1[\mathrm{~s}(43 \%)+\mathrm{d}(56 \%)]$ e $\operatorname{Pt} 2[\mathrm{~s}(43 \%)+\mathrm{d}(56 \%)]$. A segunda e a terceira ligações encontradas foram entre Pt1-Pt3 e Pt2-Pt3, ambas com as mesmas contribuições e hibridizações que a ligação citada anteriormente. Por fim, o cluster $\mathrm{Pt}_{3} \mathrm{~V}$, também apresentou a geometria piramidal triangular como a mais estável, com multiplicidade de spin igual a 6. Neste cluster temos quatro ligações: para Pt1-V4 a contribuição é exclusiva da Pt (75\%), com hibridização dos orbitais s e d de ambos os átomos, sendo esta maior no átomo de Vanádio; Temos uma ligação Pt2-Pt3, quase com a mesma contribuição dos átomos (54\% e 46\%, respectivamente), com hibridização também semelhantes $\left(\mathrm{s}^{58 \%}+\mathrm{d}^{41 \%}\right)$. Temos uma ligação com distorção de densidade bem evidente sobre o átomo de Pt em Pt2-V4, sendo $81 \%$ de Pt2 $[s(37 \%)+d(63 \%)]$ e $18 \%$ de $V 4[s(30 \%)+d(68 \%)] ;$ A última ligação para a estrutura 
é Pt3-V4, também com participação majoritária do átomo de Pt 82\% Pt3 [s(371\%) + $d(63 \%)]$ e $18 \%$ de V4 [s(29\%)+ d(69\%).

\subsection{Otimização e Propriedades Eletrônicas dos Clusters $\mathrm{Pt}_{5}$ e $\mathrm{Pt}_{4} \mathrm{M}$ (M=Al, $\mathrm{Cr}$ e V)}

$\mathrm{Na}$ Figura 7 estão apresentadas as geometrias encontradas para as estruturas de menor energia dos clusters $\mathrm{Pt}_{5}, \mathrm{Pt}_{4} \mathrm{Al}, \mathrm{Pt}_{4} \mathrm{Cr}_{\text {e }} \mathrm{Pt}_{4} \mathrm{~V}$ otimizadas com o método B3LYP/LANL2DZ e na Tabela 5, estão reunidas informações sobre distâncias interatômicas, multiplicidades de spin, configurações eletrônicas dos átomos, potenciais verticais de ionização, afinidades eletrônicas. A estrutura mais estável do cluster $\mathrm{Pt}_{5}$ é singlete com geometria bipirâmide trigonal, energia total 595,738 hartree, potencial vertical de ionização $6,10 \mathrm{eV}$ e afinidade eletrônica 3,12 eV. Li e Balbuena [59] realizaram cálculos teóricos usando a metodologia B3PW91/LANL2DZ e encontraram valores de potencial de ionização e afinidade eletrônica igual a 6,67 eV e 2,72 eV, respectivamente. De acordo com a Análise dos Orbitais Naturais temos cinco ligações no cluster Pt. São elas: para Pt1 - Pt4 acontecem três ligações. A primeira com $23 \%$ de $\operatorname{Pt} 1\left[5 d_{z} 2(99 \%)\right]$ e $77 \%$ de $\operatorname{Pt} 4\left[5 d_{z} 2(99 \%)\right]$. Esta ligação é pura e do tipo $\sigma_{d-d}$; a segunda tem contribuição invertida, isto é, $77 \%$ de $\mathrm{Pt} 1[\mathrm{~d}(99 \%)]$ e $23 \%$ de $\mathrm{Pt} 4[\mathrm{~d}(99 \%)]$, com as mesmas características da anterior; a terceira tem contribuição principal de Pt4[d(98\%)] (84\%) e $16 \%$ de $\mathrm{Pt} 1[\mathrm{~s}(88 \%)+\mathrm{d}(11 \%)]$, formando uma ligação $\mathrm{d}-\mathrm{d}$; A quarta ligação do cluster é Pt2 - Pt5, cuja natureza é 43\% de Pt2[s(6\%) + d(94\%)] e 57\% de Pt5[s(2\%) + d(98\%)], A última ligação é entre Pt2 e Pt5, onde também ocorre contribuição invertida. 
$\mathrm{O}$ estado fundamental encontrado para $\mathrm{Pt}_{4} \mathrm{Al}$ é quarteto com geometria pirâmide de base quadrada, energia total $-478,667$ hartree, potencial vertical de ionização $7,03 \mathrm{eV}$ e afinidade eletrônica $3,28 \mathrm{eV}$. No cluster $\mathrm{PtAl}_{4}$ temos três ligações para o spin $\alpha$ : Em Pt1 - Al temos $57 \%$ de Pt [6s(95\%)+ 5dz $2(5 \%)]$ e $43 \%$ de $\mathrm{Al}\left[3 \mathrm{~s}(39 \%)+3 \mathrm{p}_{\mathrm{z}}(61 \%)\right]$. Na ligação Pt2 - Al as contribuições são de $57 \%$ de Pt2[6s(95\%)+5 $\left.d_{z} 2(5 \%)\right]$ e $43 \%$ de Al[3s(39\%) + 3p $\left.(61 \%)\right]$. Em Pt3 - Al temos $60 \%$ de $\operatorname{Pt} 3\left[6 s(95 \%)+5 d_{z} 2(5 \%)\right]$ e $40 \%$ de Al[3s $\left.(19 \%)+3 p_{z}(81 \%)\right]$. Todas estas ligações são do tipo $\sigma_{s-p}$ com maior contribuição dos átomos de Platina. Onde, os orbitais $6 \mathrm{~s}$ destes átomos contribuem com mais de $90 \%$ nas ligações, enquanto que o átomo de $\mathrm{Al}$, minoritário nas ligações, contribui bastante com seus orbitais $3 p_{z}$. Para o spin $\beta$ temos 06 ligações. A primeira, entre os átomos de Pt1 e Pt3, forma hibridização com contribuição de orbitais naturais de $44 \%$ de $\mathrm{Pt} 1\left[6 \mathrm{~s}(31 \%)+5 \mathrm{p}_{\mathrm{z}}(1 \%)+5 \mathrm{~d}_{\mathrm{z}^{2}}(68 \%)\right]$ e $53 \%$ de Pt3[6s $\left.(13 \%)+5 p_{z}(1 \%)+5 d_{z} 2(86 \%)\right]$. As ligações Pt1 - Pt4, Pt2-Pt3 e Pt2Pt4 são ligações idênticas a Pt1-Pt3. As ligações entre Pt1 - Al e Pt2-Al são idênticas com contribuições de $71 \%$ de Pt1[6s(37\%) $\left.+5 d_{z_{2}}(62 \%)\right]$ com $29 \%$ de $\operatorname{Al}\left[3 s(49 \%)+3 p_{z}(51 \%)\right]$.
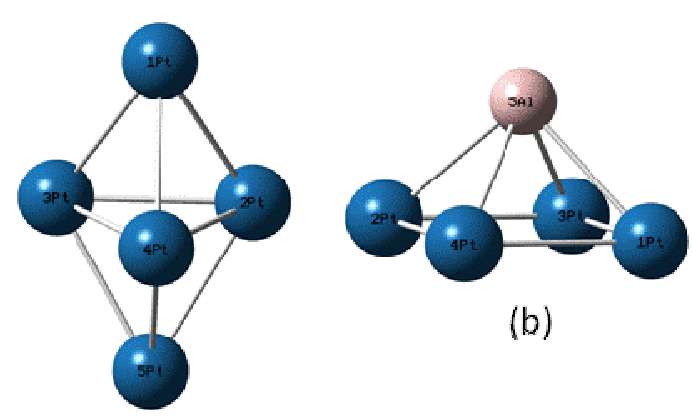

(b)

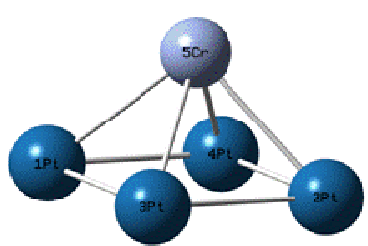

(c)

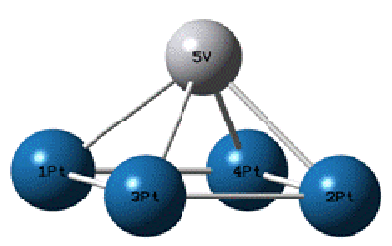

(d)

(a)

Figura 7: Geometrias das estruturas (a) $\mathrm{Pt}_{5}$, (b) $\mathrm{Pt}_{4} \mathrm{Al}$, (c) $\mathrm{Pt}_{4} \mathrm{Cr}$ e (d) $\mathrm{Pt}_{4} \mathrm{~V}$ otimizadas com o método B3LYP/LANL2DZ. 
O estado fundamental encontrado para $\mathrm{Pt}_{4} \mathrm{Cr}$ é septeto com geometria pirâmide de base quadrada, energia total $-562,890$ hartree, potencial vertical de ionização 4,68 eV e afinidade eletrônica 2,95 eV. Somente no spin a temos ligações envolvendo o átomo de $\mathrm{Cr}$. A única ligação $\mathrm{Pt}-\mathrm{Pt}$ acontece entre os átomos de números 1 e 2. Estes formam uma ligação $\sigma$ essencialmente com contribuição dos orbitais 6 s de cada átomo $(96,7 \%)$ numa ligação de contribuição de $50 \%$ para cada átomo. Em Pt3 - Cr e Pt4 - Cr os átomos de Pt são majoritários na ligação $(77,5 \%)$, formando ligações $\sigma_{s}$ - d com contribuições de $\operatorname{Pt}[6 s(94 \%)+$ $\left.5 d_{x y}(5 \%)\right]$ e $\operatorname{Cr}\left[4 s(43 \%)+3 d_{z} 2(53 \%)\right]$. No spin $\beta$ só existem ligações entre os átomos de Platina. São sete ligações $\mathrm{Pt}$ - Pt com contribuições de $50 \%$ para cada átomo na maioria das ligações (a percentagem de contribuição pode variar em cerca de 1,7\% para mais ou para menos). Todas as ligações Pt1-Pt3, Pt1-Pt4, Pt2-Pt3 e Pt2-Pt4 são idênticas e envolvem hibridização entre os orbitais $6 s$ e $5 d_{x y}$ dos átomos de $\mathrm{Pt}$ com $50 \%$ de de contribuição $\operatorname{Pt}\left[6 s(45 \%)+5 d_{x y}(54 \%)\right]$. Além dessas ligações, observamos que as ligações Pt1-Pt2 e Pt3-Pt4 são idênticas e envolvem basicamente os orbitais $5 d_{x y}$, com $50 \%$ de contribuição de cada átomo de platina.

$\mathrm{O}$ estado fundamental encontrado para $\mathrm{Pt}_{4} \mathrm{~V}$ é sexteto com geometria pirâmide de base quadrada, energia total $-548,005$ hartree, potencial vertical de ionização 7,54 eV e afinidade eletrônica 2,0 eV. Análises de NBO mostraram que No spin a temos todos os átomos de Pt ligando-se a V. Em Pt1 - Pt2 temos uma ligação uma ligação $\sigma_{\mathrm{s}-\mathrm{s}}$ ocorrendo hibridização com contribuições atômicas iguais sendo $\left[6 s(67 \%)+5 d_{x y}(33 \%)\right]$. Para Pt1 - V as contribuições são de $74 \%$ de $\operatorname{Pt}\left[6 s(28 \%)+5 d_{x y}(71 \%)\right]$ e $26 \%$ de $V\left[4 s(24 \%)+3 d_{x y}(74 \%)\right]$, formando uma ligação do tipo $\pi_{d}-d$ localizada entre os planos $x$ e y. Em Pt2 - V, Pt3 - V e Pt4 - V as hibridizações e ligações são as mesmas que na ligação Pt1 - V. Nas ligações Pt - 
Pt temos para Pt3 - Pt4 duas ligações $\sigma \mathrm{s}-\mathrm{s}$ iguais com participação de $50 \%$ de cada átomo $\left[6 \mathrm{~s}(67 \%)+5 \mathrm{~d}_{\mathrm{xy}}(33 \%)\right]$. Nota-se aqui uma participação bem mais expressiva dos átomos de $\mathrm{Pt}$ nas ligações $\mathrm{Pt}-\mathrm{V}$, o que resulta na formação de ligações do tipo $d-d$. No spin $\beta$ foram encontradas 04 ligações Pt - Pt: Pt1 - Pt3, Pt1 - Pt4, Pt2 - Pt3 e Pt2 - Pt4. Todas estas ligações apresentam a seguinte contribuição $43 \%\left[6 \mathrm{~s}(25 \%)+5 d_{x y}(74 \%)\right]$ e $57 \%\left[6 s(6 \%)+5 d_{x y}(93 \%)\right]$, respectivamente. Todas são ligações $\pi_{d-d}$ localizadas entre os planos $x$ e y. Pt2 - V e Pt1 - $V$ também apresentaram ligações $\pi_{d}-d$ sobre os eixos $x$ e y com contribuição de $81 \%$ dos átomos de Pt[6s $\left.(46 \%)+5 d_{x y}(54 \%)\right]$ e $19 \%$ de $V[4 s(13 \%)+$ $\left.3 d_{x y}(85 \%)\right]$.

Tabela 5: Distâncias interatômicas, propriedades energéticas, multiplicidade de spin, cargas atômicas de Mulliken e configurações eletrônicas dos átomos presentes nos clusters $\mathrm{Pt}_{5}, \mathrm{Pt}_{4} \mathrm{Al}, \mathrm{Pt}_{4} \mathrm{Cr}$ e $\mathrm{Pt}_{4} \mathrm{~V}$.

\begin{tabular}{|c|c|c|c|c|c|c|}
\hline Cluster & $\mathbf{m}$ & $d_{M-M}(A)$ & $Q_{M}(e)$ & Configuração eletrônica & E.A.(eV) & E.I.(eV) \\
\hline $\mathrm{Pt}_{5}$ & 1 & $\begin{array}{l}1-2=2,631 \\
1-3=2,631 \\
1-4=2,631 \\
2-3=2,619 \\
2-4=2,619 \\
3-4=2,619 \\
2-5=2,631 \\
3-5=2,631 \\
4-5=2,631\end{array}$ & $\begin{array}{l}\mathrm{Pt}_{1}=-0,040 \\
\mathrm{Pt}_{2}=+0,030 \\
\mathrm{Pt}_{3}=+0,030 \\
\mathrm{Pt}_{4}=+0,020 \\
\mathrm{Pt}_{5}=-0,040\end{array}$ & $\begin{array}{l}\mathrm{Pt}_{1} \text { [core] } 6 \mathrm{~S}^{0.73} 5 \mathrm{~d}^{9.30} 6 \mathrm{p}^{0.03} 6 \mathrm{~d}^{0.01} \\
\mathrm{Pt}_{2} \text { [core] } 6 \mathrm{~S}^{0.02} 5 \mathrm{~d}^{9.26} 6 \mathrm{p}^{0.04} 6 \mathrm{~d}^{0.01} \\
\mathrm{Pt}_{3} \text { [core] } 6 \mathrm{~S}^{0.02} 5 \mathrm{~d}^{9.26} 6 \mathrm{p}^{0.04} 6 \mathrm{~d}^{0.01} \\
\mathrm{Pt}_{4} \text { [core] } 6 \mathrm{~S}^{0.02} 5 \mathrm{~d}^{9.37} 6 \mathrm{p}^{0.04} 6 \mathrm{~d}^{0.01} \\
\mathrm{Pt}_{5} \text { [core] } 6 \mathrm{~S}^{.73} 5 \mathrm{~d}^{9.30} 6 \mathrm{p}^{0.03} 6 \mathrm{~d}^{0.01}\end{array}$ & 3,12 & 6,10 \\
\hline $\mathrm{Pt}_{4} \mathrm{Al}$ & 4 & $\begin{array}{l}1-3=2,677 \\
1-4=2,677 \\
1-5=2,558 \\
2-3=2,677 \\
2-4=2,677 \\
2-5=2,558 \\
3-5=2,558 \\
4-5=2,558\end{array}$ & $\begin{array}{l}\mathrm{Pt}_{1}=-0,256 \\
\mathrm{Pt}_{2}=-0,256 \\
\mathrm{Pt}_{3}=-0,256 \\
\mathrm{Pt}_{4}=-0,256 \\
\mathrm{Al}_{5}=+1,026\end{array}$ & $\begin{array}{l}\mathrm{Pt}_{1}[\text { core }] 6 \mathrm{~S}^{0.86} 5 \mathrm{~d}^{9.23} 6 \mathrm{p}^{0.03} \\
\mathrm{Pt}_{2}\left[\text { core } 6 \mathrm{~S}^{0.86} 5 \mathrm{~d}^{9.23} 6 \mathrm{p}^{0.03}\right. \\
\mathrm{Pt}_{3}[\text { core }] 6 \mathrm{~S}^{0.86} 5 \mathrm{~d}^{9.23} 6 \mathrm{p}^{0.03} \\
\mathrm{Pt}_{4}[\text { core }] 6 \mathrm{~S}^{0.86} 5 \mathrm{~d}^{9.23} 6 \mathrm{p}^{0.03} \\
\mathrm{Al}_{5}[\text { core }] 3 \mathrm{~S}^{1.21} 3 \mathrm{p}^{1.27} 4 \mathrm{p}^{0.02}\end{array}$ & 3,28 & 7,03 \\
\hline $\mathrm{Pt}_{4} \mathrm{Cr}$ & 7 & $\begin{array}{l}1-3=2,706 \\
1-4=2,706 \\
1-5=2,592 \\
2-3=2,706 \\
2-4=2,706 \\
2-5=2,592 \\
3-5=2,592 \\
4-5=2,592\end{array}$ & $\begin{array}{l}\mathrm{Pt}_{1}=-0,186 \\
\mathrm{Pt}_{2}=-0,186 \\
\mathrm{Pt}_{3}=-0,196 \\
\mathrm{Pt}_{4}=-0,196 \\
\mathrm{Cr}_{5}=+0,765\end{array}$ & $\begin{array}{l}\mathrm{Pt}_{1}[\text { core }] 6 \mathrm{~S}^{1.12} 5 \mathrm{~d}^{9.05} 6 \mathrm{p}^{0.03} \\
\mathrm{Pt}_{2}[\text { core }] 6 \mathrm{~S}^{1.12} 5 \mathrm{~d}^{9.05} 6 \mathrm{p}^{0.03} \\
\mathrm{Pt}_{3}[\text { core }] 6 \mathrm{~S}^{1.07} 5 \mathrm{~d}^{9.07} 6 \mathrm{p}^{0.03} \\
\mathrm{Pt}_{4}[\text { core }] 6 \mathrm{~S}^{1.07} 5 \mathrm{~d}^{9.07} 6 \mathrm{p}^{0.03} \\
\mathrm{Cr}_{5}[\text { core }] 4 \mathrm{~S}^{0.42} 3 \mathrm{~d}^{4.78} 4 \mathrm{p}^{0.05} 4 \mathrm{~d}^{0.01}\end{array}$ & 2,95 & 4,68 \\
\hline $\mathrm{Pt}_{4} \mathrm{~V}$ & 6 & $\begin{array}{l}1-3=2,677 \\
1-4=2,677 \\
1-5=2,461 \\
2-3=2,677 \\
2-4=2,677 \\
2-5=2,461\end{array}$ & $\begin{array}{l}\mathrm{Pt}_{1}=-0,184 \\
\mathrm{Pt}_{2}=-0,184 \\
\mathrm{Pt}_{3}=-0,184 \\
\mathrm{Pt}_{4}=-0,184 \\
\mathrm{~V}_{5}=+0,735\end{array}$ & $\begin{array}{l}\mathrm{Pt}_{1}[\text { core }] 6 \mathrm{~S}^{1.06} 5 \mathrm{~d}^{9.10} 6 \mathrm{p}^{0.03} \\
\mathrm{Pt}_{2}[\text { core }] 6 \mathrm{~S}^{1.00} 5 \mathrm{~d}^{9.10} 6 \mathrm{p}^{0.03} \\
\mathrm{Pt}_{3}[\text { core }] 6 \mathrm{~S}^{1.00} 5 \mathrm{~d}^{9.10} \mathrm{p}^{0.03} \\
\mathrm{Pt}_{4}\left[\text { core] } 6 \mathrm{~S}^{1.00} 5 \mathrm{~d}^{9.110} 6 \mathrm{p}^{0.03}\right. \\
\mathrm{V}_{5}[\text { core }] 4 \mathrm{~S}^{0.48} 3 \mathrm{~d}^{3.69} 4 \mathrm{p}^{0.07} 5 \mathrm{~S}^{0.01} 4 \mathrm{~d}^{0.02}\end{array}$ & 2,01 & 7,54 \\
\hline
\end{tabular}




\subsection{Interação de Oxigênio Molecular com Clusters $\mathrm{Pt}_{2}, \mathrm{Pt}-\mathrm{Al}, \mathrm{Pt}-\mathrm{Cr}, \mathrm{Pt}-\mathrm{V}$.}

Como já foi abordado anteriormente, existem três modelos pelas quais as moléculas de oxigênio podem se adsorver na superfície do eletrodo, sendo então conhecidas como modelos de Griffith, Pauling e Ponte (Yeager) [9-11]. Sendo assim, as diferentes possibilidades de adsorção foram testadas como entrada para os cálculos de interação de oxigênio molecular com as estruturas otimizadas dos clusters bimetálicos. Para a adsorção em ponte (também conhecido como modelo Yeager), duas espécies negativas têm sido identificadas experimentalmente [69-72]. Esses adsorbatos carregados negativamente possuem papel fundamental como intermediários da reação de redução de oxigênio. Um deles é o superóxido $\left(\mathrm{O}_{2}{ }^{\delta-}\right)$ caracterizado por uma frequência de estiramento da ligação $\mathrm{O}-\mathrm{O}$ na escala de $v=870-875 \mathrm{~cm}^{-1}[69,70]$. A outra espécie é peróxido $\left(\mathrm{O}_{2}^{2 \delta-}\right)$, que pode ser caracterizado por freqüência de estiramento da ligação $\mathrm{O}-\mathrm{O}$ na escala de $v=700$ $710 \mathrm{~cm}^{-1}[69,70]$. Para o superóxido a ordem de ligação da ligação O—O é estimada como sendo 1,5 e para a espécie peróxido como sendo 1,0 [69]. Na Figura 8 são apresentadas as estruturas otimizadas para a adsorção de oxigênio molecular sobre os clusters $\mathrm{Pt}_{2}$, Pt-Al, Pt-Cr e Pt-V e na Tabela 6 estão resumidos os valores de cargas atômicas de Mulliken, multiplicidades de spin e freqüências vibracionais, extraídas dos espectros teóricos de infravermelho calculados com B3LYP/LANL2DZ, 6-311G** e mostrados na Figura 9, para ligação $\mathrm{O}-\mathrm{O}$ das estruturas otimizadas $\mathrm{Pt}_{2}\left(\mathrm{O}_{2}\right)_{\text {ads }}, \mathrm{Pt}-\mathrm{Al}\left(\mathrm{O}_{2}\right)_{\text {ads }}, \mathrm{Pt}-\mathrm{Cr}\left(\mathrm{O}_{2}\right)_{\text {ads }}$ e $\mathrm{Pt}-\mathrm{V}\left(\mathrm{O}_{2}\right)_{\text {ads. }}$ Os resultados obtidos mostraram que o estado fundamental para $\mathrm{Pt}_{2}\left(\mathrm{O}_{2}\right)$ ads é singlete, onde ocorre a adsorção 
acompanhada de dissociação da molécula de oxigênio, com cada átomo de oxigênio ligado simetricamente aos átomos de platina com distância $\mathrm{Pt}-\mathrm{O}$ igual a 1,95 A. A ligação $O=O$ no oxigênio molecular tem comprimento igual a $1,21 \AA$ e frequência vibracional $1626,00 \mathrm{~cm}^{-1}$ enquanto que no $\mathrm{Pt}_{2}\left(\mathrm{O}_{2}\right)$ ads à distância interatômica $\mathrm{O}-\mathrm{O}$ passa a ser de $1,403 \AA$ com freqüência vibracional de $810,24 \mathrm{~cm}^{-1}$ e com carga de Mulliken igual a $-0,278$. Esses dados podem caracterizar o estado superóxido para o oxigênio adsorvido, como intermediário da reação de redução de oxigênio em $\mathrm{Pt}_{2}$. Além disso a ligação $\mathrm{Pt}-\mathrm{Pt}$ sofre relaxação após a adsorção de oxigênio e seu comprimento aumenta em $0,20 \AA$. Para estrutura $\mathrm{Pt}-\mathrm{Cr}\left(\mathrm{O}_{2}\right)_{\text {ads }} \mathrm{O}$ estado fundamental é quinteto, e a adsorção de oxigênio molecular também ocorre acompanhada de dissociação da molécula de oxigênio. Entretanto, os átomos de oxigênio estão ligado assimetricamente aos átomos de $\mathrm{Pt}$ e $\mathrm{Cr}$, com distância $\mathrm{Pt}-\mathrm{O}$ igual a 1,96 $\AA$ e ângulo O-Pt- $\mathrm{Cr}$ de $69,7^{\circ}$ enquanto que a distância $\mathrm{Cr}-\mathrm{O}$ encontrada foi de $1,84 \AA$ com ângulo O-Cr-Pt igual a $78,5^{\circ}$. A distância interatômica $\mathrm{O}-\mathrm{O}$ passa a ser de 1,44 $\AA$, com freqüência vibracional de $811,61 \mathrm{~cm}^{-1}$ e com carga de Mulliken igual a $-0,392$ para o átomo de oxigênio ligado ao $\mathrm{Cr}$ e -0,315 para o átomo de oxigênio ligado a $\mathrm{Pt}$. Esses dados podem caracterizar o estado peróxido para o oxigênio adsorvido na estrutura $\mathrm{Pt}-\mathrm{Cr}\left(\mathrm{O}_{2}\right)$ ads como intermediário da reação de redução de oxigênio. Observamos também uma relaxação na ligação $\mathrm{Pt}-\mathrm{Cr}$, após a interação com oxigênio molecular, onde o comprimento de ligação sofre um aumento de $0,12 \AA$.

$\mathrm{Na}$ estrutura $\mathrm{Pt}-\mathrm{V}\left(\mathrm{O}_{2}\right)_{\text {ads }}$ o oxigênio molecular também sofre adsorção dissociativa e o estado fundamental encontrado foi o quarteto. Assim como na estrutura Pt$\mathrm{Cr}\left(\mathrm{O}_{2}\right)_{\text {ads }}$, os átomos de oxigênio ligam-se assimetricamente aos átomos de $\mathrm{Pt}$ e $\mathrm{V}$, com distância $\mathrm{Pt}$-O igual a 1,96 Å e ângulo O-Pt-V 69,0 enquanto que a distância V-O encontrada foi de $1,79 \AA$ com ângulo O-V-Pt igual a $78,6^{\circ}$. O comprimento da 


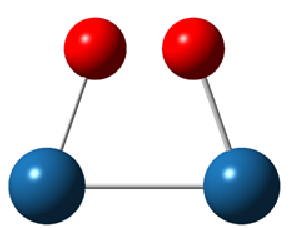

(a)

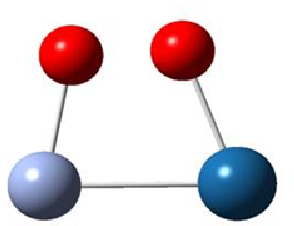

(b)

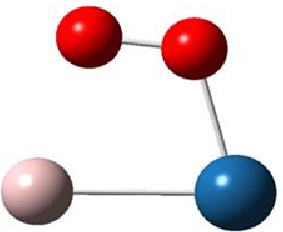

(c)

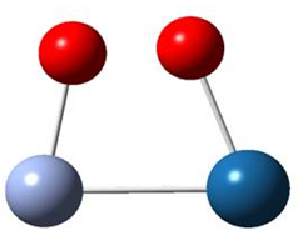

(d)

Figura 8: Geometrias das estruturas (a) $\mathrm{Pt}_{2}\left(\mathrm{O}_{2}\right)_{\text {ads }}$, (b) $\mathrm{Pt}-\mathrm{Cr}\left(\mathrm{O}_{2}\right)_{\text {ads }}$, (c) $\mathrm{Pt}-\mathrm{Al}\left(\mathrm{O}_{2}\right)_{\text {ads }}$ e (d) $\mathrm{Pt}-\mathrm{V}\left(\mathrm{O}_{2}\right)_{\text {ads }}$ otimizadas com o método B3LYP/LANL2DZ, 6-311G**.
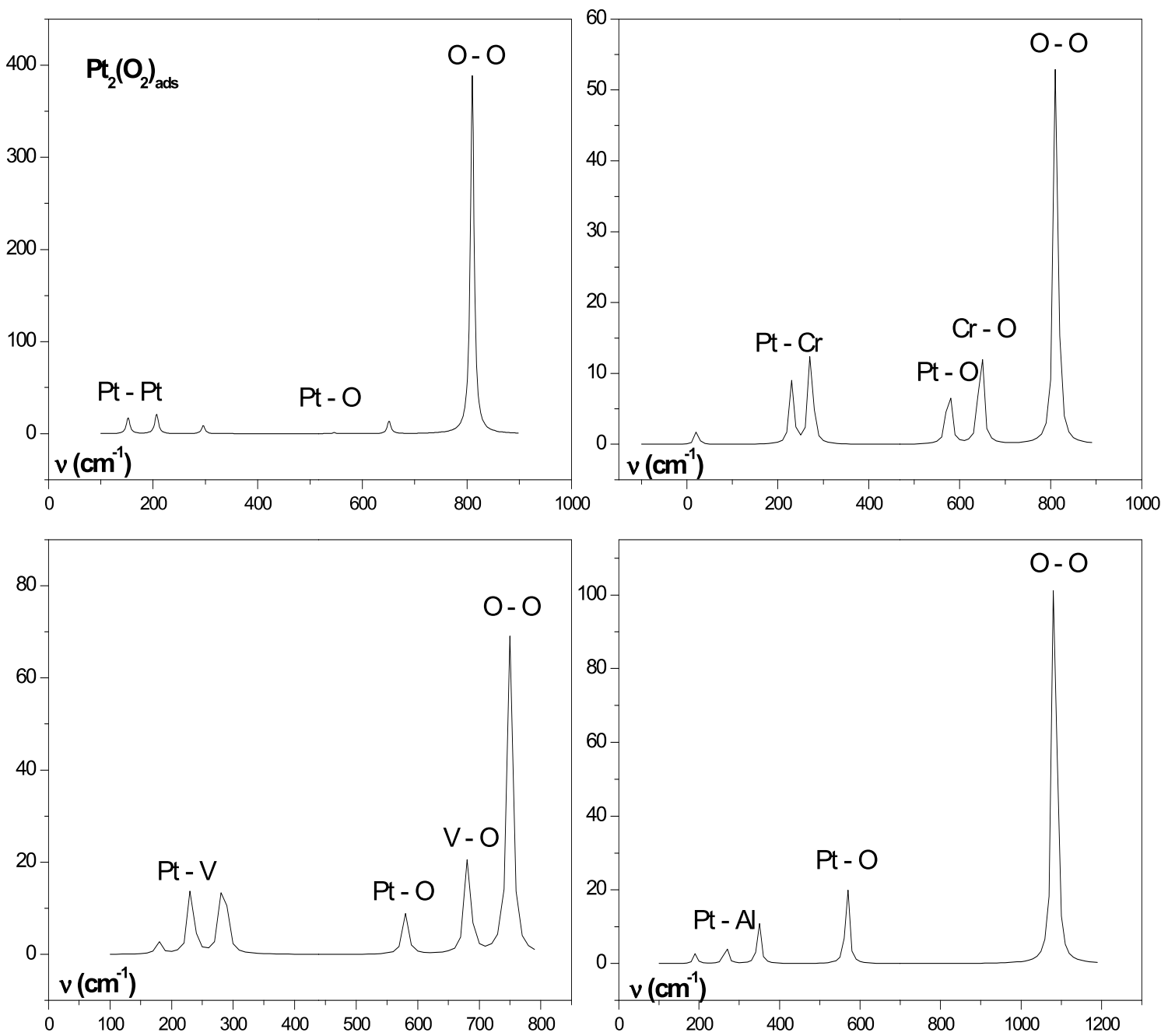

Figura 9: Espectros Teóricos de Infravermelho para as estruturas (a) $\mathrm{Pt}_{2}\left(\mathrm{O}_{2}\right)_{\text {ads }}$, (b) $\mathrm{Pt}-\mathrm{Cr}\left(\mathrm{O}_{2}\right)_{\text {ads }}$, (c) $\mathrm{Pt}-\mathrm{Al}\left(\mathrm{O}_{2}\right)_{\text {ads }}$ e (d) $\mathrm{Pt}-\mathrm{V}\left(\mathrm{O}_{2}\right)_{\text {ads }}$ [B3LYP/LANL2DZ, 6-311G ${ }^{* *}$. 
ligação $\mathrm{Pt}-\mathrm{V}$ sofre aumento de $0,20 \AA$ e a distância interatômica $\mathrm{O}-\mathrm{O}$ é $1,44 \AA$, com freqüência vibracional de $749,93 \mathrm{~cm}^{-1}$ e com carga de Mulliken igual a $-0,381$ para o átomo de oxigênio ligado ao $\mathrm{V}$ e $-0,313$ para o átomo de oxigênio ligado a Pt. Esses resultados podem caracterizar o estado peróxido para o oxigênio adsorvido na estrutura $\mathrm{Pt}-\mathrm{V}\left(\mathrm{O}_{2}\right)_{\text {ads }}$.

Tabela 6: Multiplicidades de spin, cargas atômicas de Mulliken e freqüências vibracionais da ligação $\mathrm{O}-\mathrm{O}$ das estruturas otimizadas $\mathrm{Pt}_{2}\left(\mathrm{O}_{2}\right)_{\text {ads }}$, Pt-Al $\left(\mathrm{O}_{2}\right)_{\text {ads }}$, Pt$\mathrm{Cr}\left(\mathrm{O}_{2}\right)_{\text {ads }}$ e Pt-V $\left(\mathrm{O}_{2}\right)_{\text {ads }}$ com o Método B3LYP/LANL2DZ, 6-311G**.

$\begin{array}{ccccccc}\text { Estrutura } & \mathbf{M} & \mathbf{q}_{\mathbf{P t}}(\mathbf{e}) & \mathbf{q}_{\mathbf{M}}(\mathbf{e}) & \mathbf{q}_{\mathbf{O}(3)}(\mathbf{e}) & \mathbf{q}_{\mathbf{O}(4)}(\mathbf{e}) & \mathbf{v}_{\mathbf{O}--\mathrm{o}}\left(\mathbf{c m}^{-1}\right) \\ & & & & & & \\ \mathrm{Pt}_{2}\left(\mathrm{O}_{2}\right)_{\text {ads }} & 1 & +0,278 & +0,278 & -0,278 & -0,278 & 810,24 \\ \mathrm{Pt}-\mathrm{Al}\left(\mathrm{O}_{2}\right)_{\text {ads }} & 2 & -0,266 & +0,738 & -0,188 & -0,284 & 1083,54 \\ \mathrm{Pt}-\mathrm{Cr}\left(\mathrm{O}_{2}\right)_{\text {ads }} & 5 & -0,134 & +0,841 & -0,392 & -0,315 & 811,61 \\ \mathrm{Pt}-\mathrm{V}\left(\mathrm{O}_{2}\right)_{\text {ads }} & 4 & -0,141 & +0,836 & -0,381 & -0,313 & 749,93\end{array}$

Por outro lado, a geometria otimizada para a estrutura $\mathrm{Pt}-\mathrm{Al}\left(\mathrm{O}_{2}\right)_{\mathrm{ads}}$ mostrou que a adsorção de oxigênio molecular no cluster Pt-Al segue o modelo Pauling, com multiplicidade de spin 2, onde a adsorção ocorre em apenas um sítio catalítico, que foi o átomo de platina, formando um ângulo de ligação Pt-O-O igual a $111,53^{\circ}$, distância de ligação $\mathrm{Pt}-\mathrm{O}$ 1,98 Å. Esse valor está próximo do calculado para a interação entre átomo de platina e oxigênio molecular, onde a estrutura encontrada foi semelhante a do modelo Pauling, com distância Pt—O aproximadamente a 1,95 $\AA$ e ângulo Pt-O-O aproximadamente $119^{\circ}$. Além disso, após a adsorção o comprimento de ligação $\mathrm{O}-\mathrm{O}$ aumentou em $0,11 \AA$, quando comparado com o comprimento da ligação $O=O$, com freqüência de estiramento da ligação $1083,54 \AA$ e o comprimento da ligação Pt—Al após a adsorção aumentou em 0,18 A. A carga calculada para o átomo de oxigênio ligado ao átomo Pt foi -0,188 e -0,284 para o outro átomo de oxigênio. 
A Tabela 7 apresenta as cargas atômicas e resultados das análises populacionais de Mulliken para as estruturas $\mathrm{Pt}_{2}\left(\mathrm{O}_{2}\right)_{\text {ads }}, \mathrm{Pt}-\mathrm{Al}\left(\mathrm{O}_{2}\right)_{\text {ads }}, \mathrm{Pt}-\mathrm{Cr}\left(\mathrm{O}_{2}\right)_{\text {ads }}$ e $\mathrm{Pt}-\mathrm{V}\left(\mathrm{O}_{2}\right)_{\text {ads. }}$ A distribuição da população eletrônica para os estados do oxigênio molecular ou atômico adsorvido fornece outras informações acerca do aumento no comprimento da ligação $\mathrm{O}-\mathrm{O}$ resultando ou não na quebra da ligação e no estado final do adsorbato encontrado, como sendo peróxido ou superóxido. Para efeito de comparação as populações dos orbitais das estruturas antes da interação também foram colocadas. As cargas atômicas de Mulliken mostram novamente um fluxo de carga no sentido dos clusters para os átomos de oxigênio. Nos resultados obtidos para $\mathrm{Pt}_{2}\left(\mathrm{O}_{2}\right)_{\text {ads }}$ as populações dos orbitais $\mathrm{p}$ dos átomos $\mathrm{Pt}$ são um pouco maiores após a interação com oxigênio. Além disso, podemos observar um decréscimo considerável na população dos orbitais s da platina. Em ambos os casos o átomo de Pt tem participação minoritária na ligação (32\%). Em Pt1 - O4 as contribuições são de $\operatorname{Pt}\left[6 s(26 \%)+5 d_{x}^{2}-y^{2}(74 \%)\right]$ e $O\left[2 s(5 \%)+2 p_{x}(95 \%)\right]$. Em Pt2 - O3 as contribuições são de mesma natureza. São ligações $\sigma_{d}-p$, pois os átomos de $\mathrm{Pt}$ contribuem com seus orbitais $5 d_{x}{ }^{2}-y^{2}$. Na ligação $O$ - O existe a formação de uma ligação $\pi$ com $87 \%$ de contribuição dos orbitais $p_{x}$ de cada átomo de Oxigênio. Notase aqui que há um grande aumento na participação dos orbitais $6 \mathrm{~s}$ de $\mathrm{Pt}$, concomitante a uma diminuição de contribuição dos orbitais $5 \mathrm{~d}$. Consequentemente as populações dos orbitais $p$ dos átomos de oxigênio aumentam consideravelmente. Sendo assim, podemos inferir que elétrons são transferidos do orbital s da platina para os orbitais $p$ do oxigênio. Essa transferência resulta no preenchimento dos orbitais antiligantes $\left(\pi_{\mathrm{g}}{ }^{*}\right)$ da molécula de oxigênio, causando a quebra da ligação $\mathrm{O}-\mathrm{O}$ e formando as ligações hibridizadas entre $\mathrm{Pt}-\mathrm{O}$. Os resultados obtidos para $\mathrm{Pt}-\mathrm{Cr}\left(\mathrm{O}_{2}\right)_{\text {ads }}$ e $\mathrm{Pt}-\mathrm{V}\left(\mathrm{O}_{2}\right)_{\text {ads }}$ e apresentaram comportamentos semelhantes. A 
transferência de carga e a interação ocorre de forma assimétrica e as populações dos orbitais $\mathrm{p}$ dos átomos $\mathrm{Pt}$ e $\mathrm{M}(\mathrm{M}=\mathrm{Cr}$ e $\mathrm{V})$ são um pouco maiores após a interação com oxigênio. Além disso, podemos observar um decréscimo na população dos orbitais s e d da $\mathrm{Pt}$ e do segundo metal $\mathrm{M}(\mathrm{M}=\mathrm{Cr}$ e $\mathrm{V})$, sendo que nos orbitais s a diminuição é maior quando comparado com as populações dos orbitais de Pt-M ( $\mathrm{M}=\mathrm{Cr}$ e $\mathrm{V})$. Consequentemente as populações dos orbitais $p$ dos átomos de oxigênio aumentaram, de forma assimétrica, resultando em cargas atômicas diferentes entre os átomos de oxigênio ligados com os diferentes metais. Para a estrutura $\mathrm{Pt}-\mathrm{Cr}\left(\mathrm{O}_{2}\right)_{\text {ads }}$, na ligação entre $\mathrm{Pt}$ e $\mathrm{Cr}$ a principal contribuição é dos átomos de $\operatorname{Pt}$ com 65\%[s(68\%) + d(30\%)] e 35\% de $\operatorname{Cr}[\mathbf{s}(75 \%)+d(23 \%)]$ e a ligação Pt1 O4 sendo $32 \%$ de $\mathrm{Pt} 1[\mathrm{~s}(24 \%)+\mathrm{d}(75 \%)]$ e $68 \%$ de $\mathrm{O} 4[\mathrm{~s}(6 \%)+\mathrm{p}(94 \%)]$, formando uma ligação $d-p$; Entre $\mathrm{Cr}$ e o outro oxigênio a ligação é formada com contribuições de $25 \%$ para $\operatorname{Cr} 2[\mathrm{~s}(20 \%)+\mathrm{d}(80 \%)]$ e $75 \%$ de O3[s(4\%) + p(96\%)], formando outra ligação $d-p$; Temos ainda a ligação $O$ - O com $50 \%$ de densidade para cada átomo, com formação de hibridização [s(11\%) + p(89\%)]; Estas ligações todas para o spin alfa. No spin beta $\mathrm{Pt}-\mathrm{Cr}$ observou-se a contribuição de $81 \%$ de $\operatorname{Pt}[\mathbf{s}(67 \%)+d(32 \%)]$ e $19 \%$ de $\operatorname{Cr}[s(66 \%)+d(32 \%)]$; Para Pt $-O$ temos $32 \%$ de $\operatorname{Pt}[s(25 \%)+d(75 \%)]$ e $68 \%$ de O[s(7\%) + p(93\%)]; Em Cr - O a maior contribuição é do átomo de oxigênio ( $88 \%$ ) com $91 \%$ de seus orbitais $2 p$ envolvidos juntos a uma contribuição de $12 \%$ de $\operatorname{Cr}[s(16 \%)+d(84 \%)]$; Por último, no spin beta, temos a ligação $\mathrm{O}-\mathrm{O}$ de contribuições iguais e $88 \%$ dos orbitais $2 p_{z}$ de cada átomo. $\mathrm{Na}$ interação do cluster PtV com $\mathrm{O}_{2}$ tivemos a formação de cinco ligações no spin alfa. São elas: entre Pt e V a participação principal é da Pt com 73\%[s(63\%) + d(36\%)] e $27 \%$ de $\mathrm{V}[\mathrm{s}(65 \%)+\mathrm{d}(33 \%)]$ formando uma ligação $\sigma$ entre estes átomos; Em Pt - O 
Tabela 7: Cargas atômicas e resultados das análises populacionais de Mulliken para as estruturas $\mathrm{Pt}_{2}$, Pt-Al, Pt-Cr, Pt-V [B3LYP/LANL2DZ], O $\mathrm{O}_{2}$ [B3LYP/6-311G**], $\mathrm{Pt}_{2}\left(\mathrm{O}_{2}\right)_{\text {ads }}, \mathrm{Pt}-\mathrm{Al}\left(\mathrm{O}_{2}\right)_{\text {ads }}, \mathrm{Pt}-\mathrm{Cr}\left(\mathrm{O}_{2}\right)_{\text {ads }}$ e Pt-V $\left(\mathrm{O}_{2}\right)_{\text {ads }}$ [B3LYP/LANL2DZ, 6-311G**].

\begin{tabular}{|c|c|c|c|c|c|}
\hline Estrutura & & $\operatorname{Pt}(1)$ & $\operatorname{Pt}(2)$ & $\mathrm{O}(3)$ & $\mathrm{O}(4)$ \\
\hline \multirow[t]{4}{*}{$\mathrm{Pt}_{2}$} & Carga & 0 & 0 & - & - \\
\hline & $S$ & 3,01 & 3,01 & - & - \\
\hline & $P$ & 6,06 & 6,06 & - & - \\
\hline & $\mathrm{D}$ & 8,92 & 8,92 & - & - \\
\hline \multirow[t]{4}{*}{$\mathrm{O}_{2}$} & Carga & - & - & 0 & 0 \\
\hline & $\mathrm{S}$ & - & - & 3,93 & 3,93 \\
\hline & $P$ & - & - & 4,04 & 4,04 \\
\hline & D & - & - & 0,03 & 0,03 \\
\hline \multirow{4}{*}{$\mathrm{Pt}_{2}\left(\mathrm{O}_{2}\right)_{\mathrm{ads}}$} & Carga & 0,278 & 0,278 & $-0,278$ & $-0,278$ \\
\hline & $S$ & 2,65 & 2,65 & 3,95 & 3,95 \\
\hline & $\mathrm{P}$ & 6,12 & 6,12 & 4,30 & 4,30 \\
\hline & D & 8,95 & 8,95 & 0,02 & 0,02 \\
\hline Estrutura & & $\mathrm{Pt}(2)$ & $\mathrm{Al}(1)$ & $\mathrm{O}(3)$ & $\mathrm{O}(4)$ \\
\hline \multirow[t]{4}{*}{ Pt-Al } & Carga & 0 & 0 & - & - \\
\hline & $S$ & 2,96 & 1,65 & - & - \\
\hline & $\mathrm{P}$ & 6,12 & 0,77 & - & - \\
\hline & $D$ & 9,50 & 0 & - & - \\
\hline \multirow[t]{4}{*}{$\mathrm{O}_{2}$} & Carga & - & - & 0 & 0 \\
\hline & $\mathrm{s}$ & - & - & 3,93 & 3,93 \\
\hline & $P$ & - & - & 4,04 & 4,04 \\
\hline & D & - & - & 0,03 & 0,03 \\
\hline \multirow[t]{4}{*}{$\mathrm{Pt}-\mathrm{Al}\left(\mathrm{O}_{2}\right)_{\text {ads }}$} & Carga & $-0,266$ & $+0,738$ & $-0,188$ & $-0,284$ \\
\hline & $S$ & 2,99 & 1,69 & 3,94 & 3,93 \\
\hline & $P$ & 6,17 & 0,57 & 4,22 & 4,33 \\
\hline & D & 9,08 & 0 & 0,022 & 0,02 \\
\hline Estrutura & & $\mathrm{Pt}(1)$ & $\mathrm{Cr}(2)$ & $\mathrm{O}(3)$ & $\mathrm{O}(4)$ \\
\hline \multirow[t]{4}{*}{$\mathrm{Pt}-\mathrm{Cr}$} & Carga & 0 & 0 & - & - \\
\hline & $\mathrm{S}$ & 3,43 & 2,62 & - & - \\
\hline & $\mathrm{P}$ & 6,06 & 6,02 & - & - \\
\hline & D & 9,05 & 4,81 & - & - \\
\hline \multirow{4}{*}{$\mathrm{O}_{2}$} & Carga & - & - & 0 & 0 \\
\hline & $S$ & - & - & 3,93 & 3,93 \\
\hline & $P$ & - & - & 4,04 & 4,04 \\
\hline & $D$ & - & - & 0,03 & 0,03 \\
\hline \multirow[t]{4}{*}{$\mathrm{Pt}-\mathrm{Cr}\left(\mathrm{O}_{2}\right)_{\text {ads }}$} & Carga & $-0,134$ & $+0,841$ & $-0,392$ & $-0,315$ \\
\hline & $S$ & 3,02 & 2,33 & 3,96 & 3,93 \\
\hline & $P$ & 6,14 & 6,11 & 4,34 & 4,45 \\
\hline & $\mathrm{D}$ & 8,98 & 4,72 & 0,01 & 0,01 \\
\hline Estrutura & & $\operatorname{Pt}(1)$ & $V(2)$ & $\mathrm{O}(3)$ & $\mathrm{O}(4)$ \\
\hline \multirow[t]{4}{*}{ Pt-V } & Carga & 0 & 0 & - & - \\
\hline & $S$ & 3,25 & 2,71 & - & - \\
\hline & $P$ & 6,06 & 6,01 & - & - \\
\hline & $\mathrm{D}$ & 9,23 & 3,93 & - & - \\
\hline \multirow[t]{4}{*}{$\mathrm{O}_{2}$} & Carga & - & - & 0 & 0 \\
\hline & S & - & - & 3,93 & 3,93 \\
\hline & $P$ & - & - & 4,04 & 4,04 \\
\hline & $\mathrm{D}$ & - & - & 0,03 & 0,03 \\
\hline \multirow[t]{4}{*}{ Pt-V $\left(\mathrm{O}_{2}\right)_{\text {ads }}$} & Carga & $-0,141$ & $+0,836$ & $-0,392$ & $-0,313$ \\
\hline & $\mathrm{S}$ & 3,05 & 2,33 & 3,92 & 3,93 \\
\hline & $P$ & 6,13 & 6,09 & 4,33 & 4,43 \\
\hline & D & 8,96 & 3,73 & 0,02 & 0,01 \\
\hline
\end{tabular}


o átomo oxigênio tem contribuição de $70 \%$ e $92 \%$ de seus orbitais $2 p$ com $30 \%$ de participação de Pt com $73 \%$ de seus orbitais d; Em $V$ - O, o átomo de oxigênio tem maior contribuição (84\%) contra $16 \%$ de $V$ numa ligação não hibridizada $(99,9 \%$ orbitais $p$ de $\mathrm{O}$; $98 \%$ orbitais d de $\mathrm{V}$ ) e outra ligação hibridizada com $16 \%$ de $V[s(25 \%)+d(75 \%)]$ e $84 \%$ de O[s(10\%) + d(90\%)]; Na ligação O - O foi observado uma ligação $\pi$ com $88 \%$ de participação dos orbitais $2 p$ e $50 \%$ de contribuição de cada átomo; No spin $\beta$ as ligações são: $P t-V$ temos $82 \%$ de $P t[s(67 \%)+d(33 \%)]$ e $18 \%$ de $\mathrm{V}[\mathrm{s}(65 \%)+\mathrm{d}(33 \%)]$ também formando uma ligação hibridizada; Em Pt-O a participação principal é do átomo $\mathrm{O}(69 \%)$ formando uma ligação com hibridização mais acentuada em Pt[s(25\%) $+d(75 \%)]$; Entre $\vee$ e 0 , o calcogênio tem maior contribuição eletrônica (88\%) numa ligação hibridizada; Por último, na ligação O - O temos contribuições iguais de $50 \%$ de cada átomo com formação de ligação nodal $\left(\pi_{p-p}\right)$. Estas foram as ligações no spin beta. Em ambos os casos a interação entre $\mathrm{O}_{2}$ e $\mathrm{Pt}-\mathrm{M}(\mathrm{M}=\mathrm{Cr}$ e $\mathrm{V})$ resulta no preenchimento dos orbitais antiligantes $\left(\pi_{\mathrm{g}}{ }^{*}\right)$ da molécula de oxigênio, causando a quebra da ligação $\mathrm{O}-\mathrm{O}$ e formando a ligações hibridizadas $\mathrm{Pt}-\mathrm{O}$ e $\mathrm{M}-\mathrm{O}$.

Por outro lado, no Pt-Al $\left(\mathrm{O}_{2}\right)_{\text {ads, }}$, que apresentou estrutura semelhante ao modelo de Pauling para oxigênio adsorvido, a transferência de carga ocorre principalmente dos orbitais $d$ da platina para o orbital $p$ do oxigênio, e do orbital $p$ do alumínio para o orbital d da platina. As análises de NBO revelaram que no spin $\alpha$ em $\mathrm{Al}$ - Pt o átomo de Pt tem maior participação com 85\% [6s(58\%) + 5d $\left.d_{x y}(42 \%)\right]$ e 16\% de $\operatorname{Al}\left[4 \mathrm{~s}(1,5 \%)+3 p_{z}(98,5 \%)\right]$. Esta é uma ligação $\sigma$ estendendo-se no eixo $z$ dos planos cartesianos. Pt2 - O3 forma uma ligação $\pi$ com os orbitais $5 d_{x y}$ da Pt (63\%) e os orbitais $p_{z}$ do Oxigênio (90\%) numa ligação com contribuição majoritária do átomo de Oxigênio (78\%). A ligação O - O é do tipo m (83\% de $p_{z}$ O3/87\% de $p_{z}$ O4) com 
contribuições iguais de cada átomo. No spin $\beta$ a ligação $\mathrm{Pt}-\mathrm{Al}$ também ocorre com maior participação $(83,6 \%)$ do átomo de $\mathrm{Pt}\left[6 \mathrm{~s}(60 \%)+5 \mathrm{~d}_{\mathrm{xy}}(40 \%)\right]$ e $16 \%$ de $\mathrm{Al}\left[3 \mathrm{p}_{z}\right.$ (98\%)], formando uma ligação ligação $\sigma$ estendendo-se no eixo z. Além disso, foram observadas duas ligações envolvendo O3-O4: A primeira foi uma ligação hibridizada $03\left[2 s(18 \%)+2 p_{z}(82 \%)\right]$ e $04\left[2 s(14 \%)+2 p_{z}(85 \%)\right] ;$ e a segunda foi uma ligação $\pi$ envolvendo o orbital $2 p_{z}(99,9 \%)$ para os dois átomos. Temos ainda uma ligação entre Pt2 - O3 com maior participação do átomo de oxigênio: 25\% de Pt2 [6s(34\%) $\left.+5 d_{x y}(66 \%)\right]$ e $75 \%$ de $O\left[2 s(9 \%)+2 p_{z}(91 \%)\right]$. Sendo assim, foi observado que essa transferência de carga não é suficiente para quebrar a ligação $\mathrm{O}-\mathrm{O}$ da molécula de oxigênio. Dessa forma, a adsorção de $\mathrm{O}_{2}$ em Pt-Al, que ocorre seguindo o modelo Pauling, favorecerá que a reação de redução de oxigênio ocorra seguindo o mecanismo $2 \mathrm{e}^{-}$, que tem como principal produto o peróxido de hidrogênio, pois neste caso não ocorre a quebra da ligação $\mathrm{O}-\mathrm{O}$.

Os resultados encontrados para as energias de ligação entre oxigênio molecular e os clusters $\mathrm{Pt}_{2}$, Pt-Al, Pt-Cr e Pt-V são apresentados na Tabela 8, onde também estão inseridos os dados termodinâmicos, tais como variação de entalpia, variação de energia livre e variação de entropia a 298,15 K e 1 atm de pressão. Podemos observar que a ligação torna-se mais forte quando um átomo de platina é substituído por outro metal. $\mathrm{O}$ valor da energia de ligação entre $\mathrm{Pt}_{2} \mathrm{e} \mathrm{O}_{2}$ foi de $-1,93$ $\mathrm{eV}$ e esse valor aumenta em 0,65 eV, 1,0 eV e 1,5 eV quando um átomo de platina é substituído por átomos de $\mathrm{Al}, \mathrm{Cr}$ e $\mathrm{V}$, respectivamente. Balbuena et al. [59] obtiveram um valor de 1,42 eV e para energia de ligação entre oxigênio molecular e o cluster Pt2 e 1,68 eV para energia de ligação entre oxigênio molecular e Pt-Cr, usando a metodologia B3PW91/LANL2DZ, 6-31G*. A adsorção de oxigênio sobre os clusters Pt-Al, Pt-Cr e Pt-V é uma reação muito exotérmica e envolve maior variação 
de entalpia e energia livre, que para a adsorção no cluster $\mathrm{Pt}_{2}$. Dentre os clusters estudados o Pt-V foi o que apresentou maior energia de adsorção e maior variação de energia livre. Além disso, todas as reações apresentaram variações negativas de entropia e não foram observadas diferenças significativas na variação de entropia para adsorção de oxigênio.

Tabela 8: Energia de ligação, variação de entalpia, variação de energia livre e variação de entropia para a reação: $\mathrm{Pt}-\mathrm{M}+\mathrm{O}_{2} \rightarrow \mathrm{Pt}-\mathrm{M}\left(\mathrm{O}_{2}\right)$ ads. Dados Termodinâmicos obtidos a 298,15 K e p=1 atm. [B3LYP/LANL2DZ, 6-311G**].

$\begin{array}{ccccc}\text { Reação } & \mathbf{E}_{\text {Ligação }}(\mathbf{e V}) & \Delta \mathbf{H}\left(\mathbf{k c a l ~ m o l}^{-1}\right) & \Delta \mathbf{G}\left(\mathbf{k c a l ~ m o l}^{-1}\right) & \Delta \mathbf{S}\left(\mathbf{c a l ~ K}^{-1} \mathbf{m o l}^{-1}\right) \\ \mathrm{Pt}_{2}+\mathrm{O}_{2} \rightarrow \mathrm{Pt}_{2}\left(\mathrm{O}_{2}\right)_{\text {ads }} & -1,93 & -45,94 & -35,74 & -32,22 \\ \mathrm{Pt}-\mathrm{Al}+\mathrm{O}_{2} \rightarrow \mathrm{Pt}-\mathrm{Al}\left(\mathrm{O}_{2}\right)_{\text {ads }} & -2,58 & -60,18 & -51,46 & -29,31 \\ \mathrm{Pt}-\mathrm{Cr}+\mathrm{O}_{2} \rightarrow \mathrm{Pt}-\mathrm{Cr}\left(\mathrm{O}_{2}\right)_{\text {ads }} & -2,93 & -68,27 & -60,65 & -30,65 \\ \mathrm{Pt}-\mathrm{V}+\mathrm{O}_{2} \rightarrow \mathrm{Pt}-\mathrm{V}\left(\mathrm{O}_{2}\right)_{\text {ads }} & -3,43 & -79,95 & -70,91 & -31,05\end{array}$

Para entender como a dissociação da ligação $\mathrm{O}$ - $\mathrm{O}$ ocorre sobre $\mathrm{Pt}_{2}$, curvas de superfície de energia potencial foram calculadas e são apresentadas na Figura 10, onde foram incluídos os valores obtidos para a distância interatômica $\mathrm{Pt}$ - O. Como mostrado na Figura 10, foi encontrado o valor de aproximadamente 1,0 eV para a barreira de dissociação da ligação $\mathrm{O}-\mathrm{O}$ adsorvido em $\mathrm{Pt}_{2}$. Eichler e Hafner [41] realizaram cálculos de DFT com funcional GGA-PW91 e também encontraram um valor de aproximadamente $1,0 \mathrm{eV}$ para a barreira de dissociação para $\mathrm{O}$ - $\mathrm{O}$ sobre superfície Pt(111). Sidik e Anderson [73] realizaram estudos de DFT com a metodologia B3LYP/LANL2DZ, 6-31G** para a redução de $\mathrm{O}_{2}$ sobre um sítio duplo de platina com distância interatômica $\mathrm{Pt}$ - Pt fixada em 2,775 $\AA$ e encontraram o valor $0,74 \mathrm{eV}$ para a barreira de dissociação de $\mathrm{O}_{2}$ e destacaram que este valor é cerca de duas vezes maior que o obtido experimentalmente por Gland et. al. [70] para alto grau de recobrimento de superfície em alto vácuo, que foi de $0,38 \mathrm{eV}$. 
Estudos realizados para a barreira de dissociação de $\mathrm{O}_{2}$ sobre $\mathrm{Pt}-\mathrm{Cr}$ e Pt-V também foram realizados e os resultados são apresentados na Figura 11, onde foi inserida a curva de dissociação de $\mathrm{O}_{2}$ adsorvido em $\mathrm{Pt}_{2}$ para efeitos de comparação, e encontramos valores de $0,56 \mathrm{eV}$ e $0,20 \mathrm{eV}$, para a barreira de dissociação de $\mathrm{O}_{2}$ sobre Pt-Cr e Pt-V, respectivamente. Esses resultados mostram que a dissociação de $\mathrm{O}_{2}$ ocorre mais facilmente sobre esses clusters que sobre o cluster $\mathrm{Pt}_{2}$.

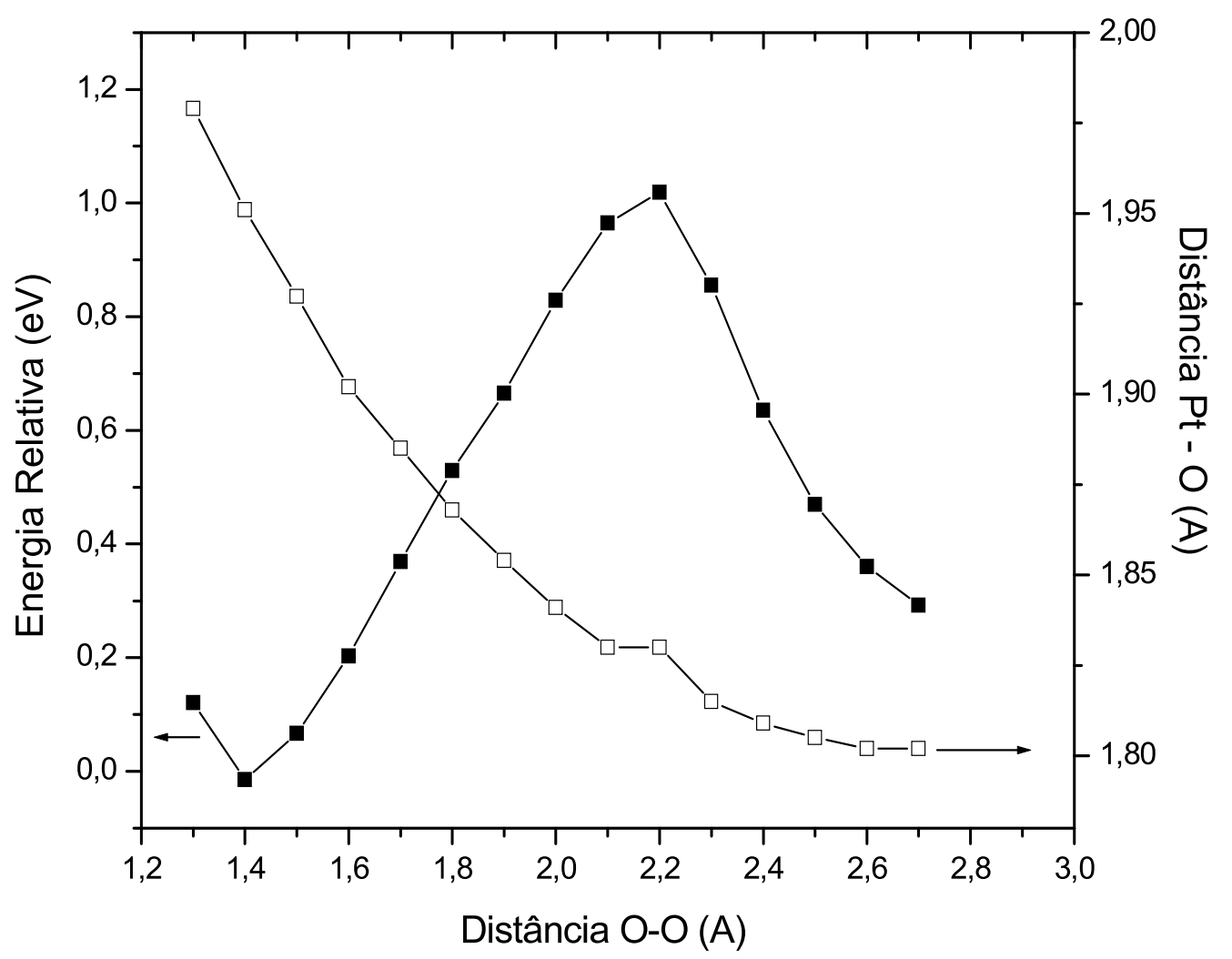

Figura 10: Curva de superfície de energia potencial com barreira energética para a dissociação de Oxigênio molecular adsorvido em $\mathrm{Pt}_{2}$ e variação no comprimento da ligação $\mathrm{Pt}-\mathrm{O}$ [B3LYP/LANL2DZ, 6-311G**]. Energias calculadas em relação às energias das estruturas otimizadas. 


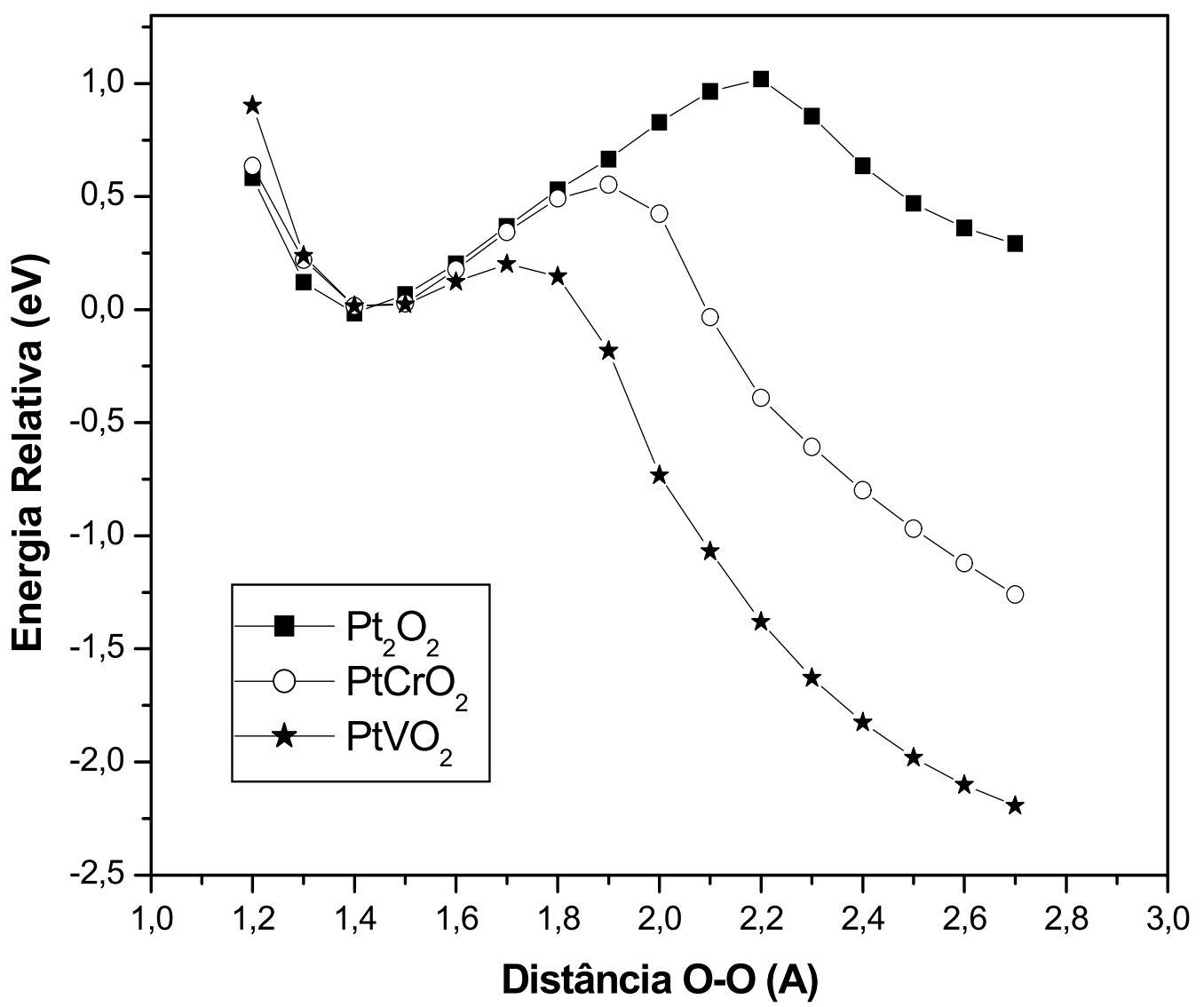

Figura 11: Curva de superfície de energia potencial com barreira energética para a dissociação de Oxigênio molecular adsorvido em $\mathrm{Pt}_{2}, \quad \mathrm{Pt}-\mathrm{Cr}$ e $\mathrm{Pt}-\mathrm{V}$ [B3LYP/LANL2DZ, 6-311G**]. Energias calculadas em relação às energias das estruturas otimizadas. 


\subsection{Interação de Oxigênio Molecular com Clusters $\mathrm{Pt}_{3}, \mathrm{Pt}_{2} \mathrm{Al}, \mathrm{Pt}_{2} \mathrm{Cr}, \mathrm{Pt}_{2} \mathrm{~V}$.}

Na Figura 12 são apresentadas as estruturas otimizadas para a adsorção de oxigênio molecular sobre os clusters $\mathrm{Pt}_{3}, \mathrm{Pt}_{2} \mathrm{Al}_{1} \mathrm{Pt}_{2} \mathrm{Cr}$ e $\mathrm{Pt}_{2} \mathrm{~V}$ e na Tabela 9 estão resumidos os valores de cargas atômicas de Mulliken, multiplicidades de spin, comprimentos de ligação, ângulos e freqüências de estiramento da ligação O—O, obtidas a partir dos espectros teóricos de infravermelho mostrados na Figura 13, das estruturas otimizadas $\mathrm{Pt}_{3}\left(\mathrm{O}_{2}\right)_{\text {ads }}, \quad \mathrm{Pt}{ }_{2} \mathrm{Al}\left(\mathrm{O}_{2}\right)_{\text {ads }}, \quad \mathrm{Pt}_{2} \mathrm{Cr}\left(\mathrm{O}_{2}\right)_{\text {ads }}$ e $\quad \mathrm{Pt}_{2} \mathrm{~V}\left(\mathrm{O}_{2}\right)_{\text {ads }}$. Os resultados obtidos mostraram que o estado fundamental para $\mathrm{Pt}_{3}\left(\mathrm{O}_{2}\right)_{\mathrm{ads}}$ é singlete, onde ocorre a adsorção não dissociativa da molécula de oxigênio. Podemos observar que após a adsorção de oxigênio molecular houve a quebra da ligação Pt1Pt2, com cada átomo de oxigênio ligado simetricamente e em ponte aos átomos e de platina com distância $\mathrm{Pt}-\mathrm{O}$ igual a $1,952 \AA$ e $\mathrm{O}-\mathrm{O}$ igual a $1,346 \AA \mathrm{com}$ frequência de estiramento $914,60 \mathrm{~cm}^{-1}$ e com cargas de Mulliken igual a -0,258 o que pode caracterizar, dessa forma, o estado superóxido para o oxigênio adsorvido, como intermediário da reação de redução de oxigênio em $\mathrm{Pt}_{3}$. Para a estrutura $\mathrm{Pt}_{2} \mathrm{Al}\left(\mathrm{O}_{2}\right)_{\text {ads }}$ mais estável é duplete, onde ocorre a adsorção dissociativa da molécula de oxigênio. Entretanto, os átomos de oxigênio estão ligados assimetricamente aos átomos de Pt e Al, com distância Pt—O igual a 2,039 A e ângulo O-Pt-Al(4-1-3) de $65,88^{\circ}$ enquanto que a distância Al—O encontrada foi de 1,795 A com ângulo O-Al$\operatorname{Pt}(5-3-1)$ igual a $85,397^{\circ}$. A distância interatômica O—O passa a ser de 1,480 $\AA$, com frequência de estiramento de $762,70 \mathrm{~cm}^{-1}$ e com carga de Mulliken igual a 0,489 para o átomo de oxigênio ligado ao $\mathrm{Al}$ e $-0,322$ para o átomo de oxigênio ligado a Pt. Esses dados podem caracterizar o estado peróxido para o oxigênio adsorvido na estrutura $\mathrm{Pt}_{2} \mathrm{Al}\left(\mathrm{O}_{2}\right)_{\text {ads }}$ como intermediário da reação de redução de oxigênio. Observamos também uma pequena relaxação na ligação $\mathrm{Pt} — \mathrm{Al}$, após a 
interação com oxigênio molecular, onde o comprimento de ligação sofre um aumento de $0,04 \AA$.

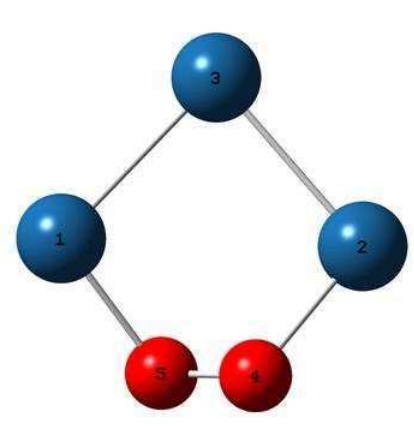

(a)

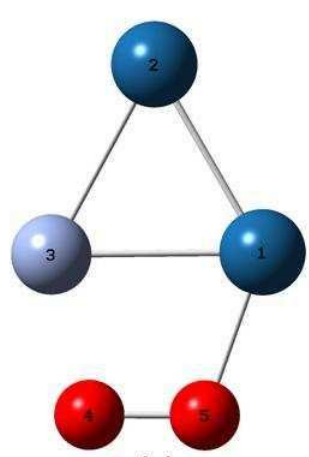

(c)

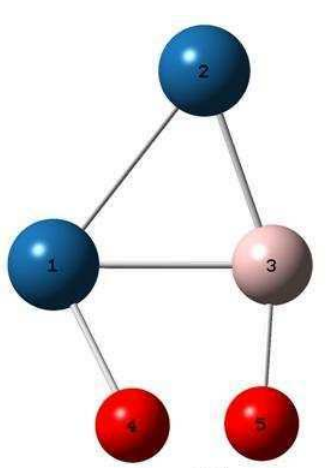

(b)

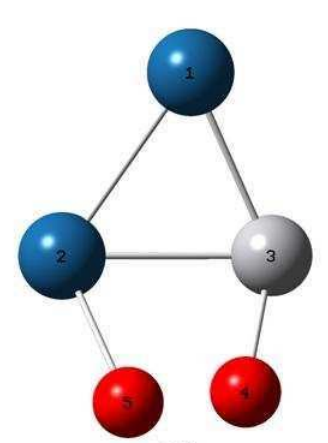

(d)

Figura 12: Geometrias das estruturas (a) $\mathrm{Pt}_{3}\left(\mathrm{O}_{2}\right)_{\text {ads }}$, (b) $\mathrm{Pt}_{2} \mathrm{Al}\left(\mathrm{O}_{2}\right)_{\text {ads }}$, (c) $\mathrm{Pt}_{2} \mathrm{Cr}\left(\mathrm{O}_{2}\right)_{\text {ads }}$ e (d) $\mathrm{Pt}_{2} \mathrm{~V}\left(\mathrm{O}_{2}\right)_{\text {ads }}$ otimizadas com o método B3LYP/LANL2DZ, 6-311G**.

Enquanto isso, para estrutura $\mathrm{Pt}_{2} \mathrm{Cr}\left(\mathrm{O}_{2}\right)_{\text {ads }}$ o estado fundamental é tripleto e observamos que a adsorção de oxigênio molecular sobre o cluster $\mathrm{Pt}_{2} \mathrm{Cr}$, não é acompanhada de dissociação da molécula de $\mathrm{O}_{2}$. A adsorção ocorre em apenas um sítio catalítico, seguindo o modelo de Pauling, que foi o átomo de platina, formando

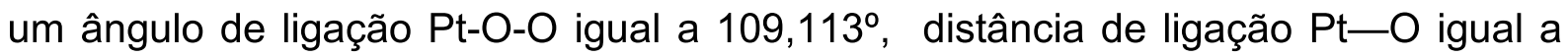
2,046 Å. Esse valor está 0,096 Å maior que o calculado para a interação entre átomo de platina e oxigênio molecular, onde a estrutura encontrada foi semelhante a do modelo Pauling, com distância $\mathrm{Pt}-\mathrm{O}$ aproximadamente a 1,95 Å. Além disso, após a adsorção o comprimento de ligação $\mathrm{O}-\mathrm{O}$ aumentou em $0,15 \AA$, quando 
comparado com o comprimento da ligação no $\mathrm{O}_{2}$ gasoso, com freqüência vibracional $933,22 \AA$. A carga obtida para o átomo de oxigênio ligado ao átomo $\mathrm{Pt}$ foi $-0,224$ (O5) e -0,312 para o outro átomo de oxigênio (O4).
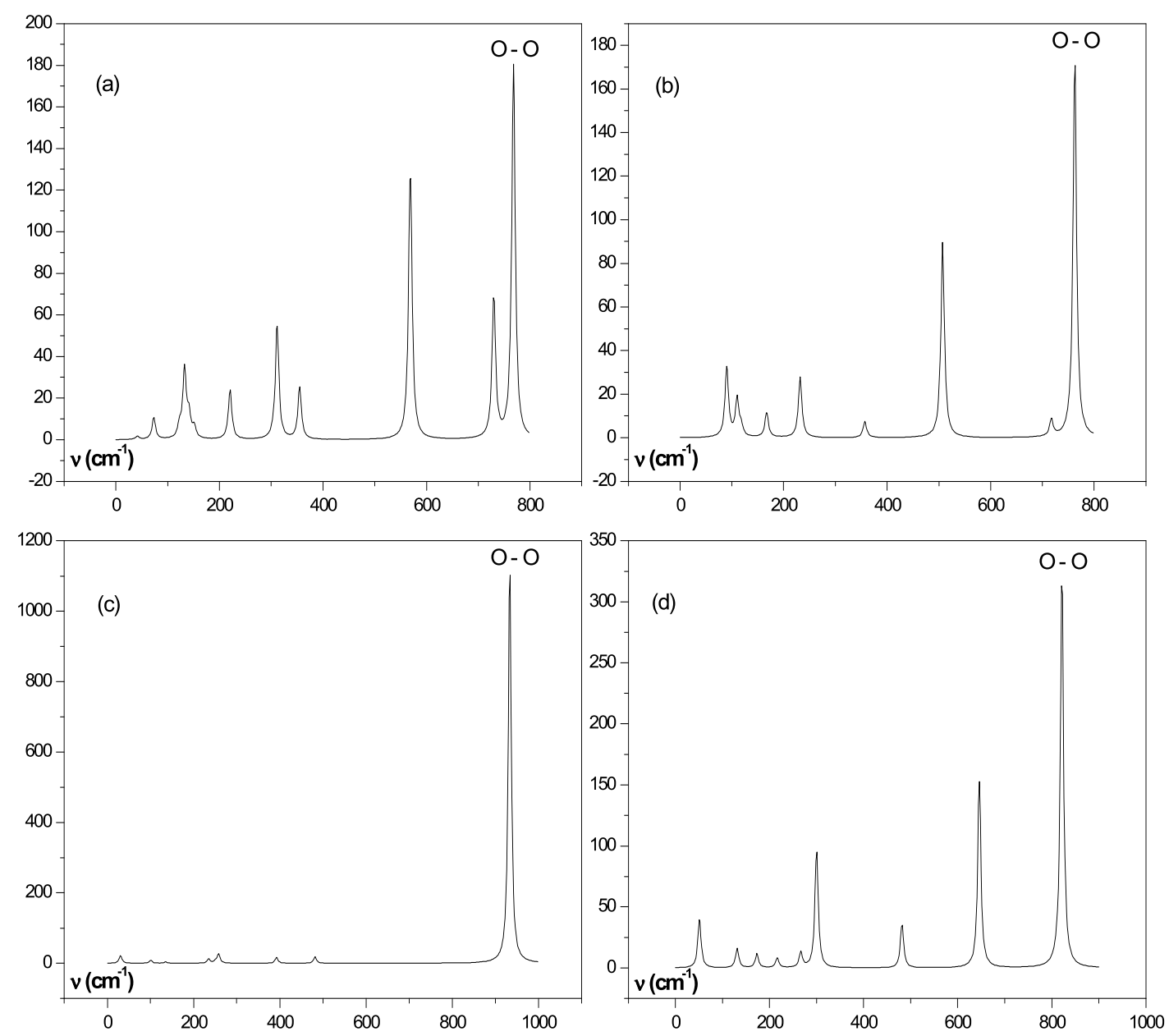

Figura 13: Espectros Teóricos de Infravermelho para as estruturas (a) $\mathrm{Pt}_{3}\left(\mathrm{O}_{2}\right)_{\text {ads }}$, (b) $\mathrm{Pt}_{2} \mathrm{Al}\left(\mathrm{O}_{2}\right)_{\text {ads }}$, (c) $\mathrm{Pt}_{2} \mathrm{Cr}\left(\mathrm{O}_{2}\right)_{\text {ads }}$ e (d) $\mathrm{Pt}_{2} \mathrm{~V}\left(\mathrm{O}_{2}\right)_{\text {ads. }}$ [B3LYP/LANL2DZ, 6-311G**]. 
Tabela 9: Distâncias interatômicas, multiplicidade de spin, cargas atômicas de Mulliken e freqüências de estiramento para $\mathrm{Pt}_{3}\left(\mathrm{O}_{2}\right)_{\text {ads }}, \mathrm{Pt}_{2} \mathrm{Al}\left(\mathrm{O}_{2}\right)_{\text {ads }}, \mathrm{Pt}_{2} \mathrm{Cr}\left(\mathrm{O}_{2}\right)_{\text {ads }} \mathrm{e}$ $\mathrm{Pt}_{2} \mathrm{~V}\left(\mathrm{O}_{2}\right)_{\text {ads. }}$.

\begin{tabular}{|c|c|c|c|c|c|}
\hline Cluster & $\mathbf{m}$ & Distâncias (A) & Cargas (e) & Ângulo ( $\left.{ }^{\circ}\right)$ & $v_{0-o}\left(\mathrm{~cm}^{-1}\right)$ \\
\hline $\mathrm{Pt}_{3}\left(\mathrm{O}_{2}\right)_{\text {ads }}$ & 1 & $\begin{array}{l}1-2=3.421 \\
1-3=2.534 \\
2-3=2.534 \\
1-5=1.952 \\
2-4=1.952 \\
4-5=1.346\end{array}$ & $\begin{array}{l}\mathrm{Pt}_{1}=+0,267 \\
\mathrm{Pt}_{2}=+0,267 \\
\mathrm{Pt}_{3}=-0,017 \\
\mathrm{O}_{4}=-0,258 \\
\mathrm{O}_{5}=-0,258\end{array}$ & $\begin{array}{l}1-3-2=84.884 \\
3-2-4=99.158 \\
2-4-5=110.29 \\
4-5-1=110.29 \\
3-1-5=99.161\end{array}$ & 914,60 \\
\hline $\mathrm{Pt}_{2} \mathrm{Al}(\mathrm{C}$ & 2 & $\begin{array}{l}1-2=2.730 \\
2-3=2.307 \\
1-3=2.440 \\
3-5=1.795 \\
1-4=2.039 \\
4-5=1.480\end{array}$ & $\begin{array}{l}\mathrm{Pt}_{1}=-0,145 \\
\mathrm{Pt}_{2}=-0,276 \\
\mathrm{Al}_{3}=+1,231 \\
\mathrm{O}_{4}=-0,322 \\
\mathrm{O}_{5}=-0,489\end{array}$ & $\begin{array}{l}1-2-3=57.205 \\
2-1-3=52.646 \\
1-3-2=70.148 \\
1-3-5=85.397 \\
3-1-4=65.882\end{array}$ & 762,70 \\
\hline $\mathrm{Pt}_{2} \mathrm{C}$ & 3 & $\begin{array}{l}1-2=2.560 \\
1-3=2.493 \\
2-3=2.554 \\
1-5=2.046 \\
4-5=1.362\end{array}$ & $\begin{array}{l}\mathrm{Pt}_{1}=-0,057 \\
\mathrm{Pt}_{2}=-0,281 \\
\mathrm{Cr}_{3}=+0,875 \\
\mathrm{O}_{4}=-0,312 \\
\mathrm{O}_{5}=-0,224\end{array}$ & $\begin{aligned} 1-2-3 & =58.352 \\
1-3-2 & =60.944 \\
2-1-3 & =60.704 \\
1-5-4 & =109.113\end{aligned}$ & 933,25 \\
\hline $\mathrm{Pt}_{2} \mathrm{~V}(\mathrm{C}$ & 4 & $\begin{array}{l}1-3=2.382 \\
1-2=2.617 \\
2-3=2.462 \\
3-4=1.800 \\
2-5=2.051 \\
4-5=2.420\end{array}$ & $\begin{array}{l}\mathrm{Pt}_{1}=-0,269 \\
\mathrm{Pt}_{2}=-0,038 \\
\mathrm{~V}_{3}=+0,948 \\
\mathrm{O}_{4}=-0,347 \\
\mathrm{O}_{5}=-0,294\end{array}$ & $\begin{array}{l}1-2-3=55.828 \\
1-3-2=65.379 \\
2-1-3=58.792 \\
2-3-4=77.163 \\
3-2-5=71.209\end{array}$ & 821,59 \\
\hline
\end{tabular}

Os cálculos realizados para a estrutura $\mathrm{Pt}_{2} \mathrm{~V}\left(\mathrm{O}_{2}\right)_{\text {ads }}$ mostraram que o estado fundamental é quarteto, e a adsorção de oxigênio molecular também foi acompanhada de dissociação da ligação $\mathrm{O}-\mathrm{O}$, onde foi observado à distância $\mathrm{Pt}$ O igual a 2,051 Å e ângulo O-Pt-V(5-3-2) de $71,209^{\circ}$ enquanto que a distância $V-O$ encontrada foi de 1,80 A com ângulo O-V-Pt(4-3-2) igual a 77,163․ A distância interatômica O-O passa a ser de 2,42 A, com freqüência vibracional de $821,59 \mathrm{~cm}^{-1}$ e com carga de Mulliken igual a -0,347 para o átomo de oxigênio ligado ao $\mathrm{V}$ e - 
0,294 para o átomo de oxigênio ligado a Pt. Esses dados podem caracterizar o estado peróxido para o oxigênio adsorvido na estrutura $\mathrm{Pt}_{2} \mathrm{~V}\left(\mathrm{O}_{2}\right)_{\text {ads }}$ como intermediário da reação de redução de oxigênio. Observamos também um grande aumento na ligação $\mathrm{Pt}-\mathrm{V}$, após a interação com oxigênio molecular, onde o comprimento de ligação sofre um aumento de 0,216 Å.

A Tabela 10 apresenta as cargas atômicas e resultados das análises populacionais de Mulliken para as estruturas $\mathrm{Pt}_{3}\left(\mathrm{O}_{2}\right)_{\text {ads }}, \mathrm{Pt}_{2} \mathrm{Al}\left(\mathrm{O}_{2}\right)_{\text {ads }}, \mathrm{Pt}_{2} \mathrm{Cr}\left(\mathrm{O}_{2}\right)_{\text {ads }}$ e $\mathrm{Pt}_{2} \mathrm{~V}\left(\mathrm{O}_{2}\right)_{\text {ads. }}$ Como já foi abordado anteriormente, essa distribuição da população eletrônica fornece outras informações acerca do aumento no comprimento da ligação $\mathrm{O}-\mathrm{O}$ resultando ou não na quebra da ligação e no estado final, peróxido ou superóxido, encontrado. Para efeito de comparação as populações de Mulliken das estruturas antes da interação também foram colocadas. As cargas atômicas de Mulliken mostram novamente um fluxo de carga no sentido dos clusters para os átomos de oxigênio. Podemos observar nos resultados obtidos para $\operatorname{Pt}_{3}\left(\mathrm{O}_{2}\right)_{\text {ads }}$ que as populações dos orbitais d dos átomos Pt1 e Pt2 diminuem após a interação com oxigênio, enquanto que as populações dos orbitais $p$ de ambos os átomos de oxigênio aumentam de 4,04 para 4,29 após a interação com o cluster $\mathrm{Pt}_{3}$. Nesta estrutura otimizada observamos que ambas as ligações Pt1-O5 e Pt2-O4, são idênticas, em cerca de $31 \%$ de participação da platina $[s(27 \%)+d(73 \%)]$ e $69 \%$ do oxigênio $[s(6.0 \%)+p(94 \%)]$, resultando numa configuração eletrônica [core] $2 S^{1.81} 2 p^{4.46} 3 S^{0.01} 3 p^{0.01}$ para os átomos de oxigênio. A transferência de elétrons dos orbitais $d$ da platina para os orbitais $p$ do oxigênio não é suficiente para haver quebra de ligação $\mathrm{O}$ - O mas provoca um alongamento e enfraquecimento da ligação $\mathrm{O}-\mathrm{O}$, resultando numa ligação O - O hibridizada $\left(\mathrm{s}^{25 \%}+\mathrm{p}^{75 \%}\right)$ com $50 \%$ de contribuição de cada átomo. 
Nos resultados para $\mathrm{Pt}_{2} \mathrm{Al}\left(\mathrm{O}_{2}\right)_{\text {ads }}$ observamos que as populações dos orbitais s e d do átomo Pt1 sofrem uma diminuição considerável enquanto que o orbital p sobre aumento nas populações. Por outro lado, para o átomo $\mathrm{Pt}_{2}$ foi observado uma diminuição na população dos orbitais s e p e um aumento considerável na população dos orbitais d. Além disso, para o átomo Al3 foi observado que as populações dos orbitais $s$ diminuem praticamente a metade enquanto que os orbitais $p$ sofrem aumento na população de Mulliken após interação com $\mathrm{O}_{2}$. Paralelamente a isso, os orbitais $\mathrm{p}$ dos átomos de oxigênio sofrem aumento nas populações, principalmente o átomo $\mathrm{O} 5$ ligado à Al3. Essa transferência eletrônica resulta no preenchimento dos orbitais antiligantes $\left(\pi_{\mathrm{g}}{ }^{*}\right)$ da molécula de oxigênio, sendo suficiente para causar a quebra da ligação $\mathrm{O}-\mathrm{O}$ e formação das ligações $\mathrm{Pt1}$-O4 e Al3-O5. Análises de NBO para a estrutura $\mathrm{Pt}_{2} \mathrm{Al}\left(\mathrm{O}_{2}\right)$ ads revelaram que no spin alfa temos múltiplas ligações, onde a primeira é Pt1 - O4 com 19\% de Pt1 [s(80\%) + d(20\%)] e $81 \%$ de O4 $[s(8 \%)+p(92 \%)]$. Foi observado também que a ligação Pt2 -Al3 é formada com $46 \%$ de Pt2 $[s(94 \%)+d(4 \%)]$ e $54 \%$ de Al3 $[s(50 \%)+p(49 \%)]$ e para a ligação Al3O5 temos menor participação dos átomos de $\mathrm{Al}$ sendo $13 \%$ de $\mathrm{Al} 3[\mathrm{~s}(48 \%)+\mathrm{p}(52 \%)]$ e $87 \%$ de $05[s(16 \%)+p(84 \%)] ;$ No spin beta temos também múltiplas ligações: em Pt1 - Pt2 temos $41.5 \%$ de Pt1 $[s(58 \%)+p(4 \%)+d(38 \%)]$ e $58.46 \%$ de Pt2 $[s(25 \%)+p(2 \%)+d(72 \%)]$, onde já percebemos grande participação dos orbitais naturais d na ligação; Em Pt1-O4 o oxigênio contribui principalmente através de seus orbitais p com contribuições de $31 \% \operatorname{Pt} 1[\mathrm{~s}(28 \%)+\mathrm{d}(71 \%)]$ e $69 \%$ de 04 $[s(5 \%)+p(95 \%)] ;$ Em Pt2 -Al3 temos 55\% de Pt2 $[s(68 \%)+p(3 \%)+d(29 \%)]$ e $45 \%$ de Al3 [s(49\%) + p(50\%)], formando ligações $\sigma$ hibridizadas; e por fim, a ligação Al3 - O5 é formada com $12 \%$ de $\mathrm{Al} 3[\mathrm{~s}(48 \%)+\mathrm{p}(52 \%)]$ e $88 \%$ de O5 $[\mathrm{s}(18 \%)+p$ $(81 \%)]$ 
Nos resultados para $\mathrm{Pt}_{2} \mathrm{Cr}\left(\mathrm{O}_{2}\right)_{\text {ads }}$ observamos que as populações dos orbitais $\mathrm{d}$ dos dois átomos Pt sofrem uma diminuições enquanto que o orbital $\mathrm{p}$ do átomo Pt1 e s do átomo Pt2 apresentaram aumentos nas populações após interação com oxigênio molecular. Além disso, a população dos orbitais s do átomo $\mathrm{Cr} 3$ diminuiu de 2,45 para 2,31 enquanto que dos orbitais d apresentaram considerável aumento de 4,01 para 4,67 após a interação com oxigênio. Por outro lado, para os átomos de oxigênio observamos acréscimos nas populações dos orbitais $p$, como resultado desta interação. Para compreender melhor as ligações existentes os resultados de NBO para os spins $\alpha$ e $\beta$ e revelaram a existência de 06 ligações no spin $\alpha$ : para Pt1-Pt2 a contribuição eletrônica de cada átomo é $72 \%$ de Pt1 $[s(22 \%)+\mathrm{d}(77 \%)]$ e 28\% de Pt2[s(25\%) + d(75\%); Para a ligação Pt1-Cr3 a contribuição do átomo de Pt é maior, sendo $75 \%$ de Pt1 $[s(53 \%)+d(45 \%)]$ e $25 \%$ de $\operatorname{Cr}[s(56 \%)+p(8 \%)+$ d(36\%)]; Para a ligação Pt1-O5 a contribuição do átomo de Pt é maior, sendo 64\% de Pt1 [d(99,4\%)] e 36\% de O5 [p(99,2\%)], formando uma ligação $\sigma_{d-p}$; Em Pt2-Cr3 temos $80 \%$ de Pt2 $[s(71 \%)+d(28 \%)]$ e $20 \%$ de $\operatorname{Cr} 3[s(30 \%)+p(53 \%)+d(17 \%)] ; 0$ oxigênio também se liga ao $\mathrm{Cr}$ na ligação $\mathrm{Cr} 3$ - O4, cujo caráter é de $10 \%$ de $\mathrm{Cr} 3$ $[s(12 \%)+p(39 \%)+d(49 \%)]$ e $90 \%$ de $04[s(6 \%)+p(93 \%)]$; A ligação O-O ocorre com simetria de overlap e orbitais s e p hibridizados, sendo que a participação dos orbitais $p$ é maior $[s(15 \%)+p(84 \%)]$. No spin $\beta$ temos duas ligações: a natureza da primeira ligação é Pt1-Pt2, onde ocorre a participação majoritária do átomo de Pt2, sendo $64 \%$ de Pt2[s(90\%) $+d(9 \%)]$ e $36 \%$ de $\operatorname{Pt} 1[s(76 \%)+p(13 \%)+d(11 \%)]$. A natureza da última ligação deste spin é a ligação O-O, que ocorre de maneira simétrica, com a contribuição de $50 \%$ de cada átomo $[s(13 \%)+p(87 \%)]$. 
Tabela 10: Cargas atômicas e resultados das análises populacionais de Mulliken para as estruturas $\mathrm{Pt}_{3}, \mathrm{Pt}_{2} \mathrm{Al}, \mathrm{Pt}_{2} \mathrm{Cr}_{2}, \mathrm{Pt}_{2} \mathrm{~V}$ [B3LYP/LANL2DZ], $\mathrm{O}_{2}$ [B3LYP/6-311G**], $\mathrm{Pt}_{3}\left(\mathrm{O}_{2}\right)_{\text {ads }}, \mathrm{Pt}_{2} \mathrm{Al}\left(\mathrm{O}_{2}\right)_{\mathrm{ads}}, \mathrm{Pt}{ }_{2} \mathrm{Cr}\left(\mathrm{O}_{2}\right)_{\mathrm{ads}}$ e $\mathrm{Pt}_{2} \mathrm{~V}\left(\mathrm{O}_{2}\right)_{\text {ads }}$ [B3LYP/LANL2DZ, 6-311G**].

\begin{tabular}{|c|c|c|c|c|c|c|}
\hline Estrutura & & $\mathrm{Pt}(1)$ & $\mathrm{Pt}(2)$ & $\mathrm{Pt}(3)$ & $\mathrm{O}(4)$ & $\mathrm{O}(5)$ \\
\hline \multirow[t]{4}{*}{$\mathrm{Pt}_{3}$} & Carga & 0,00 & 0,00 & 0,00 & - & - \\
\hline & S & 2,77 & 2,77 & 2,77 & - & - \\
\hline & $\mathrm{P}$ & 6,12 & 6,12 & 6,12 & - & - \\
\hline & $D$ & 9,12 & 9,12 & 9,12 & - & - \\
\hline \multirow[t]{4}{*}{$\mathrm{O}_{2}$} & Carga & - & - & & 0,00 & 0,00 \\
\hline & $S$ & - & - & & 3,93 & 3,93 \\
\hline & $P$ & - & - & & 4,04 & 4,04 \\
\hline & $D$ & - & - & & 0,03 & 0,03 \\
\hline \multirow{4}{*}{$\mathrm{Pt}_{3}\left(\mathrm{O}_{2}\right)_{\mathrm{ads}}$} & Carga & $+0,267$ & $+0,267$ & $-0,017$ & $-0,258$ & $-0,258$ \\
\hline & $S$ & 2,74 & 2,74 & 2,75 & 3,95 & 3,95 \\
\hline & $\mathrm{P}$ & 6,16 & 6,16 & 6,09 & 4,29 & 4,29 \\
\hline & $D$ & 8,83 & 8,83 & 9,17 & 0,02 & 0,02 \\
\hline Estrutura & & $\mathrm{Pt}(1)$ & $\mathrm{Pt}(2)$ & $\mathrm{Al}(3)$ & $\mathrm{O}(4)$ & $\mathrm{O}(5)$ \\
\hline \multirow[t]{4}{*}{$\mathrm{Pt}_{2} \mathrm{Al}$} & Carga & $-0,426$ & $-0,426$ & $+0,852$ & - & - \\
\hline & S & 3,04 & 3,04 & 1,69 & - & - \\
\hline & $\mathrm{P}$ & 6,19 & 6,19 & 0,65 & - & - \\
\hline & D & 9,08 & 9,08 & 0,00 & - & - \\
\hline \multirow[t]{4}{*}{$\mathrm{O}_{2}$} & Carga & - & - & & 0 & 0 \\
\hline & $\mathrm{s}$ & - & - & & 3,93 & 3,93 \\
\hline & $P$ & - & - & & 4,04 & 4,04 \\
\hline & D & - & - & & 0,03 & 0,03 \\
\hline \multirow[t]{4}{*}{$\mathrm{Pt}_{2} \mathrm{Al}\left(\mathrm{O}_{2}\right)_{\mathrm{ad}}$} & Carga & $-0,145$ & $-0,276$ & $+1,231$ & $-0,322$ & $-0,489$ \\
\hline & S & 2,93 & 2,84 & 0,84 & 3,96 & 3,94 \\
\hline & $P$ & 6,26 & 6,10 & 0,93 & 4,34 & 4,53 \\
\hline & D & 8,96 & 9,33 & 0,00 & 0,01 & 0,01 \\
\hline Estrutura & & $\mathrm{Pt}(1)$ & $\mathrm{Pt}(2)$ & $\mathrm{Cr}(3)$ & $\mathrm{O}(4)$ & $\mathrm{O}(5)$ \\
\hline \multirow[t]{4}{*}{$\mathrm{Pt}_{2} \mathrm{Cr}$} & Carga & $-0,318$ & $-0,318$ & $+0,637$ & - & - \\
\hline & $S$ & 2,99 & 2,99 & 2,45 & - & - \\
\hline & $\mathrm{P}$ & 6,09 & 6,09 & 6,13 & - & - \\
\hline & D & 9,23 & 9,23 & 4,01 & - & - \\
\hline \multirow[t]{4}{*}{$\mathrm{O}_{2}$} & Carga & - & - & & 0 & 0 \\
\hline & S & - & - & & 3,93 & 3,93 \\
\hline & $\mathrm{P}$ & - & - & & 4,04 & 4,04 \\
\hline & D & - & - & & 0,03 & 0,03 \\
\hline \multirow[t]{4}{*}{$\mathrm{Pt}_{2} \mathrm{Cr}\left(\mathrm{O}_{2}\right)_{\mathrm{ad}}$} & Carga & $-0,057$ & $-0,281$ & $+0,875$ & $-0,312$ & 0,224 \\
\hline & $S$ & 2,94 & 3,13 & 2,31 & 3,93 & 3,95 \\
\hline & $P$ & 6,23 & 6,04 & 6,15 & 4,37 & 4,26 \\
\hline & D & 8,88 & 9,10 & 4,67 & 0,02 & 0,02 \\
\hline \multicolumn{2}{|l|}{ Estrutura } & $\mathrm{Pt}(1)$ & $\mathrm{Pt}(2)$ & $\mathrm{V}(3)$ & $\mathrm{O}(4)$ & $\mathrm{O}(5)$ \\
\hline \multirow[t]{4}{*}{$\mathrm{Pt}_{2} \mathrm{~V}$} & Carga & $-0,371$ & $-0,371$ & $+0,742$ & - & - \\
\hline & S & 3,33 & 3,33 & 2,50 & - & - \\
\hline & $\mathrm{P}$ & 6,06 & 6,06 & 6,14 & - & - \\
\hline & $\mathrm{D}$ & 8,98 & 8,98 & 3,62 & - & - \\
\hline \multirow[t]{4}{*}{$\mathrm{O}_{2}$} & Carga & - & - & & 0 & 0 \\
\hline & S & - & - & & 3,93 & 3,93 \\
\hline & $\mathrm{P}$ & - & - & & 4,04 & 4,04 \\
\hline & D & - & - & & 0,03 & 0,03 \\
\hline \multirow[t]{4}{*}{$\mathrm{Pt}_{2} \mathrm{~V}\left(\mathrm{O}_{2}\right)_{\text {ads }}$} & Carga & $-0,269$ & $-0,038$ & $+0,948$ & $-0,347$ & $-0,294$ \\
\hline & S & 3,16 & 2,91 & 2,34 & 3,92 & 3,96 \\
\hline & $\mathrm{P}$ & 6,03 & 6,26 & 6,14 & 4,41 & 4,32 \\
\hline & $\mathrm{D}$ & 9,06 & 8,86 & 3,57 & 0,02 & 0,02 \\
\hline
\end{tabular}


Nos resultados para $\mathrm{Pt}_{2} \mathrm{~V}\left(\mathrm{O}_{2}\right)_{\text {ads }}$ observamos que as populações dos orbitais $\mathrm{s}$ do átomo Pt1 e dos orbitais s e d de Pt2 diminuem enquanto que há um aumento na população dos orbitais d de Pt1 e nos orbitais $p$ de Pt2. Com isso, podemos inferir que o fluxo de carga acontece dos orbitais s e d de Pt2 o orbital p do O5. Além disso, para o átomo V3 foi observado que as populações dos orbitais s e d diminuem após interação com $\mathrm{O}_{2}$, ocorrendo transferência de carga para os orbitais $\mathrm{p}$ do $\mathrm{O}$. Essa transferência de carga dos orbitais s e d de Pt2 e V3 resulta no preenchimento dos orbitais antiligantes $\left(\pi_{\mathrm{g}}{ }^{*}\right)$ da molécula de oxigênio, causando a quebra da ligação $\mathrm{O}-\mathrm{O}$ e formando as ligações Pt2-O5 e V3-O4. Análises de NBO mostraram que no Spin a temos a ligação Pt1-Pt2 formada com $70 \%$ de Pt1[s(58\%) + d(41\%)] e $30 \%$ de Pt2[s $(42 \%)+p(10 \%)+d(47 \%)] ;$ Em Pt1 - V3 a participação da Platina é bem maior, com $71 \%[s(38 \%)+d(62 \%)]$ e $29 \%$ de vanádio $[s(31 \%)+p(7 \%)+$ d(61\%)]. A ligação V3-O4 o átomo de oxigênio tem participação majoritária na ligação, com $83 \%$, cujo envolvimento de seus orbitais p chega a $98 \%$, enquanto que o orbital mais envolvido do Vanádio é o d (87\%). As análises para o spin $\beta$ revelaram que há um pequeno aumento (de cerca de $5 \%$ ) na contribuição da Pt para as ligações Pt-V e diminuição de $67 \%$ para cerca de $36 \%$ de participação dos orbitais d da Pt e 48\% para os átomos de V. Para este spin nota-se uma ligação Pt2-O5 com maior participação do átomo de oxigênio formando ligação Pt2-O5, com a contribuição de $32 \%$ de Pt2 $[s(17 \%)+d(83 \%)]$ e $68 \%$ de $05[s(6 \%)+p(94 \%)]$. Também foi observada uma ligação V3-O4, com contribuição de 13\% de V3 [s(6\%) + $d(92 \%)]$ e $87 \%$ de oxigênio [s(15\%) + p(85\%)].

Para obtermos as barreiras de energias de dissociação da ligação $\mathrm{O}-\mathrm{O}$ após a interação com clusters $\mathrm{Pt}_{2} \mathrm{Al}$ e $\mathrm{Pt}_{2} \mathrm{~V}$, curvas de superfície de energia potencial foram calculadas e são apresentadas na Figura 14. Como pode ser observado, foi 
encontrado o valor de aproximadamente $0,21 \mathrm{eV}$ para a barreira de dissociação da ligação $\mathrm{O}-\mathrm{O}$ adsorvido sobre o cluster $\mathrm{Pt}_{2} \mathrm{Al}$ e aproximadamente $0,30 \mathrm{eV}$ para a barreira energética de dissociação da ligação $\mathrm{O}-\mathrm{O}$ adsorvido sobre o cluster $\mathrm{Pt}_{2} \mathrm{~V}$. Esses resultados mostram que a dissociação de $\mathrm{O}_{2}$ ocorre mais facilmente sobre o cluster $\mathrm{Pt}_{2} \mathrm{Al}$ que sobre o cluster $\mathrm{Pt}_{2} \mathrm{~V}$.

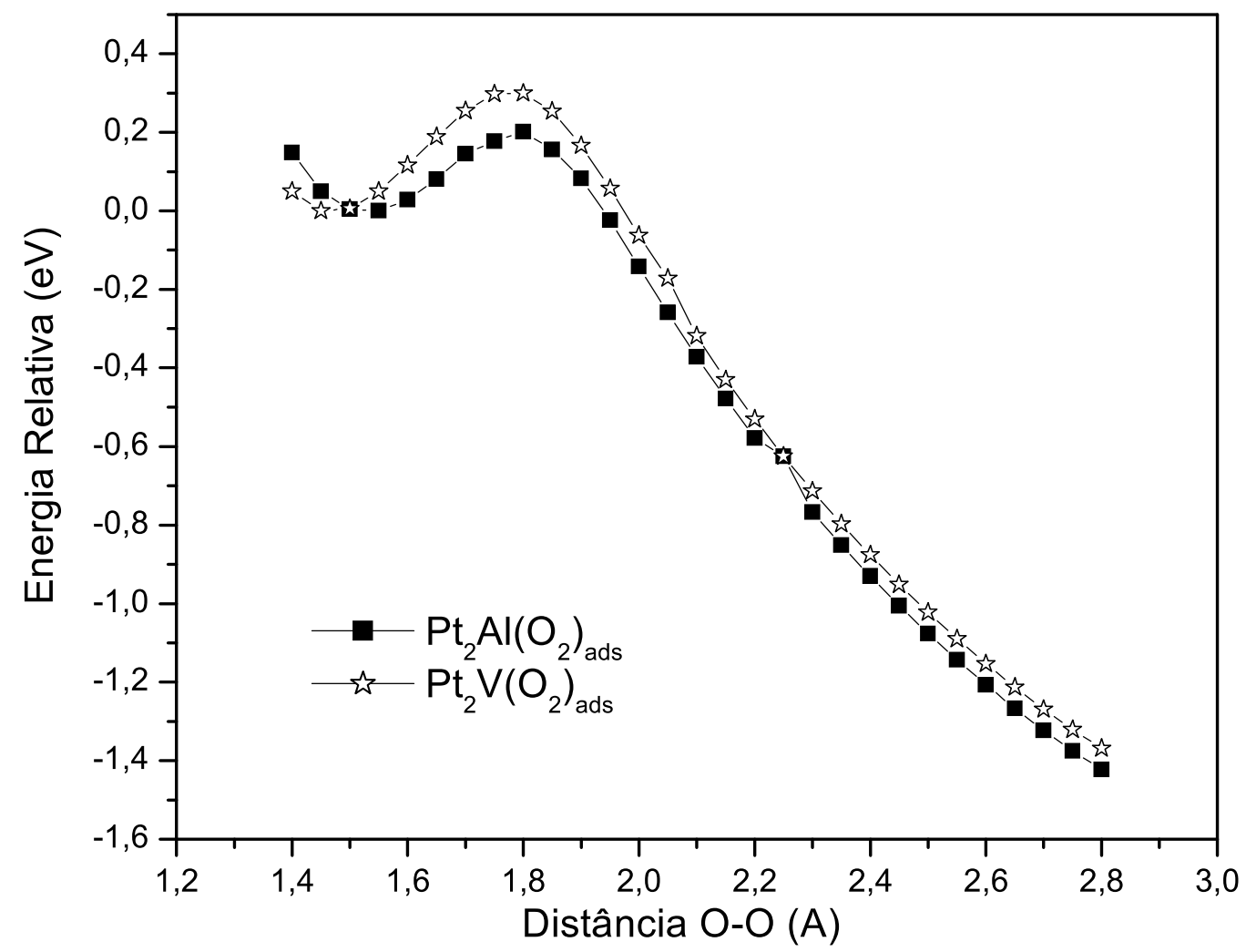

Figura 14: Curva de superfície de energia potencial com barreira energética para a dissociação de oxigênio molecular adsorvido em $\mathrm{Pt}_{2} \mathrm{Al}$ e $\mathrm{Pt}_{2} \mathrm{~V}$ [B3LYP/LANL2DZ, 6$311 \mathrm{G}^{* *}$. Energias calculadas em relação às energias das estruturas otimizadas. 
Os resultados encontrados para as energias de ligação entre oxigênio molecular e os clusters $\mathrm{Pt}_{3}, \mathrm{Pt}_{2} \mathrm{Al}, \mathrm{Pt}_{2} \mathrm{Cr}_{\text {e }} \mathrm{Pt}_{2} \mathrm{~V}$ são apresentados na Tabela 11, onde também estão inseridos os dados termodinâmicos, tais como variação de entalpia, variação de energia livre e variação de entropia a $298,15 \mathrm{~K}$ e 1 atm de pressão. Podemos observar novamente que a ligação torna-se mais forte quando um átomo de platina é substituído por outro metal. $\mathrm{O}$ valor da energia de ligação entre $\mathrm{Pt}_{3} \mathrm{e}_{2}$ foi de $-1,94 \mathrm{eV}$ e esse valor aumenta em $0.77 \mathrm{eV}, 0.94 \mathrm{eV}$ e $1.74 \mathrm{eV}$ quando um átomo de platina é substituído por átomos de $\mathrm{Al}, \mathrm{Cr}$ e $\mathrm{V}$, respectivamente. Além disso, podemos observar que a adsorção de oxigênio sobre os clusters $\mathrm{Pt}_{2} \mathrm{Al}, \mathrm{Pt}_{2} \mathrm{Cr}$ e $\mathrm{Pt}_{2} \mathrm{~V}$ é uma reação muito exotérmica e envolve maior variação de entalpia e energia livre, que para a adsorção no cluster $\mathrm{Pt}_{3}$. Dentre os clusters estudados $\mathrm{o}$ $\mathrm{Pt}_{2} \mathrm{~V}$ foi o que apresentou maior calor de adsorção e maior variação de energia livre. Além disso, todas as reações apresentaram variações negativas de entropia e não foram observadas diferenças significativas na variação de entropia para adsorção de oxigênio.

Tabela 11: Energia de ligação, variação de entalpia, variação de energia livre e variação de entropia para a reação: $\mathrm{Pt}_{2} \mathrm{M}+\mathrm{O}_{2} \rightarrow \mathrm{Pt}_{2} \mathrm{M}\left(\mathrm{O}_{2}\right)_{\text {ads }}$. Dados Termodinâmicos obtidos a 298,15 K e p=1 atm. [B3LYP/LANL2DZ, 6-311G**].

$\begin{array}{ccccc}\text { Reação } & \mathbf{E}_{\text {Ligação }}(\mathbf{e V}) & \Delta \mathbf{H}\left(\mathbf{k c a l ~ m o l}^{-1}\right) & \Delta \mathbf{G}\left(\mathbf{k c a l ~ m o l}^{-1}\right) & \Delta \mathbf{S}\left(\mathbf{c a l ~ K}^{-1} \mathbf{~ m o l}^{-1}\right) \\ \mathrm{Pt}_{3}+\mathrm{O}_{2} \rightarrow \mathrm{Pt}_{2}\left(\mathrm{O}_{2}\right)_{\text {ads }} & -1,94 & 45.49 & & \\ \mathrm{Pt}_{2} \mathrm{Al}+\mathrm{O}_{2} \rightarrow \mathrm{Pt}_{2} \mathrm{Al}\left(\mathrm{O}_{2}\right)_{\text {ads }} & -2,71 & -63,08 & -55.77 & -32,61 \\ \mathrm{Pt}_{2} \mathrm{Cr}+\mathrm{O}_{2} \rightarrow \mathrm{Pt}_{2} \mathrm{Cr}\left(\mathrm{O}_{2}\right)_{\text {ads }} & -2,88 & -66,85 & -52,65 & -34,99 \\ \mathrm{Pt}_{2} \mathrm{~V}+\mathrm{O}_{2} \rightarrow \mathrm{Pt}_{2} \mathrm{~V}\left(\mathrm{O}_{2}\right)_{\text {ads }} & -3,68 & -85,57 & -75,10 & -34,72 \\ & & & -33,59\end{array}$




\subsection{Interação de Oxigênio Molecular com Clusters $\mathrm{Pt}_{4}, \mathrm{Pt}_{3} \mathrm{Al}, \mathrm{Pt}_{3} \mathrm{Cr}_{\text {e }} \mathrm{Pt}_{3} \mathrm{~V}$.}

Na Figura 15 são apresentadas as estruturas otimizadas para a adsorção de oxigênio molecular sobre os clusters $\mathrm{Pt}_{4}, \mathrm{Pt}_{3} \mathrm{Al}, \mathrm{Pt}_{3} \mathrm{Cr}$ e $\mathrm{Pt}_{3} \mathrm{~V}$ e na Tabela 12 estão resumidos os valores de cargas atômicas de Mulliken, multiplicidades de spin, comprimentos de ligação, ângulos e freqüências de estiramento da ligação O—O, obtidas a partir dos espectros teóricos de infravermelho mostrados na Figura 16, das estruturas otimizadas $\quad \mathrm{Pt}_{4}\left(\mathrm{O}_{2}\right)_{\text {ads }}, \quad \mathrm{Pt}_{3} \mathrm{Al}\left(\mathrm{O}_{2}\right)_{\text {ads }}, \quad \operatorname{Pt} t_{3} \mathrm{Cr}\left(\mathrm{O}_{2}\right)_{\text {ads }}$ e $\quad \mathrm{Pt}_{3} \mathrm{~V}\left(\mathrm{O}_{2}\right)_{\text {ads }}$. Os resultados obtidos mostraram que o estado fundamental para $\mathrm{Pt}_{4}\left(\mathrm{O}_{2}\right)_{\mathrm{ads}}$ é singlete, onde ocorre adsorção da molécula de oxigênio sem dissociação da ligação O - O envolvendo uma relaxação na estrutura $\mathrm{Pt}_{4}$, provocando a dissociação da ligação Pt3-Pt4. As distâncias interatômicas encontradas para as ligações Pt2 - O6 e Pt3 O5 foram 1,997 e 2,041 A, respectivamente. O comprimento da ligação O - O obtido foi $1,355 \AA$, com frequência de estiramento $933,66 \mathrm{~cm}^{-1}$ e com cargas de Mulliken igual a - -242 para o átomo 05 e - $-2,255$ para o átomo 06 , o que caracteriza, dessa forma, o estado superóxido para o oxigênio adsorvido, como intermediário da reação de redução de oxigênio em $\mathrm{Pt}_{4}$. Além disso, foi observado uma diminuição no comprimento de ligação Pt2—Pt3 após a interação com oxigênio molecular de 2,363 para $2,461 \AA$.

Para estrutura $\mathrm{Pt}_{3} \mathrm{Al}\left(\mathrm{O}_{2}\right)_{\text {ads }}$ o estado fundamental é duplete, onde foi observada a adsorção dissociativa da molécula de oxigênio produzindo uma relaxação na estrutura $\mathrm{Pt}_{4} \mathrm{Al}$ e resultando, assim, na dissociação da ligação Pt1-Pt3. Entretanto, os átomos de oxigênio estão ligado assimetricamente aos átomos de $\mathrm{Pt}$ e Al, com distância Pt3-O6 igual a 1,966 A e ângulo O-Pt-Al(6-3-4) de 67,143 enquanto que a distância Al4-O5 encontrada foi de 1,798 A com ângulo O-Al-Pt(5- 
4-3) igual a $79,828^{\circ}$. A distância interatômica O—O passa a ser de $1,477 \AA$, com freqüência vibracional de $768,19 \mathrm{~cm}^{-1}$ e com carga de Mulliken igual a $-0,491$ para o átomo de oxigênio ligado ao $\mathrm{Al}$ e $-0,311$ para o átomo de oxigênio ligado a Pt. Esses dados podem caracterizar o estado peróxido para o oxigênio adsorvido na estrutura $\mathrm{Pt}_{3} \mathrm{Al}\left(\mathrm{O}_{2}\right)_{\text {ads }}$ como intermediário da reação de redução de oxigênio. Observamos, também, uma pequena mudança no comprimento de ligação Pt3-Al4, onde o comprimento de ligação sofre uma diminuição de $0,146 \AA$.

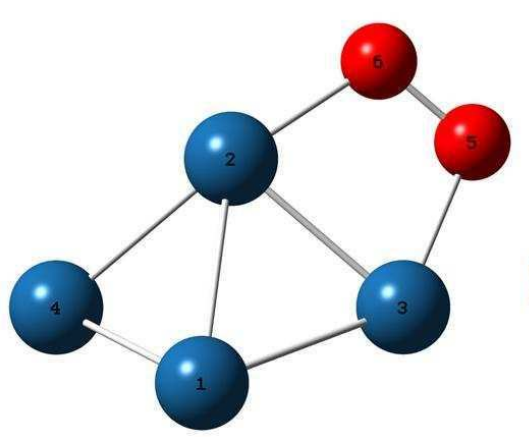

(a)

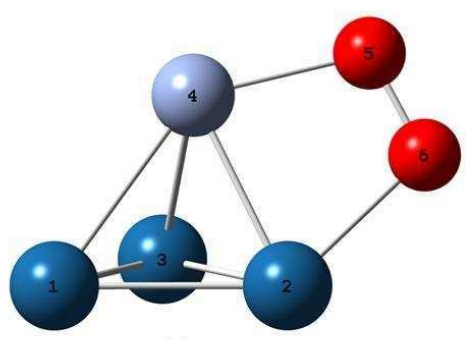

(c)

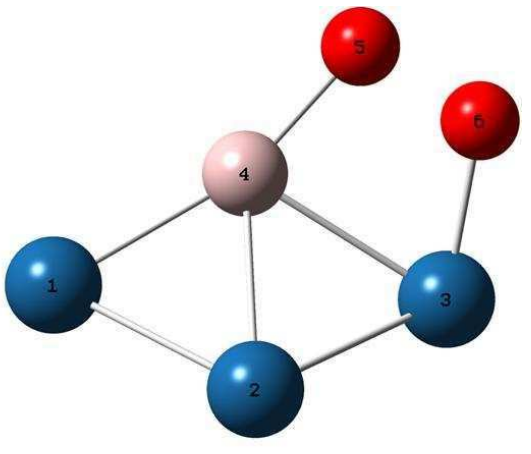

(b)

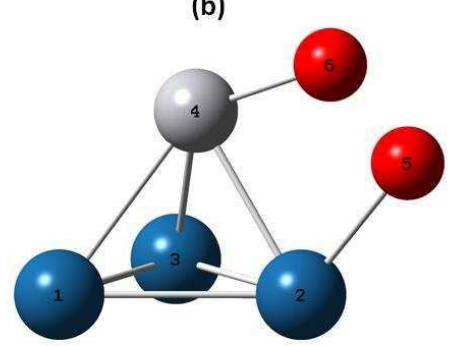

(d)

Figura 15: Geometrias das estruturas (a) $\mathrm{Pt}_{4}\left(\mathrm{O}_{2}\right)_{\text {ads }}$, (b) $\mathrm{Pt}_{3} \mathrm{Al}\left(\mathrm{O}_{2}\right)_{\text {ads }}$, (c) $\mathrm{Pt}{ }_{3} \mathrm{Cr}\left(\mathrm{O}_{2}\right)_{\text {ads }}$ e (d) $\mathrm{Pt}_{3} V\left(\mathrm{O}_{2}\right)_{\text {ads }}$ otimizadas com o método B3LYP/LANL2DZ, 6-311G**. 

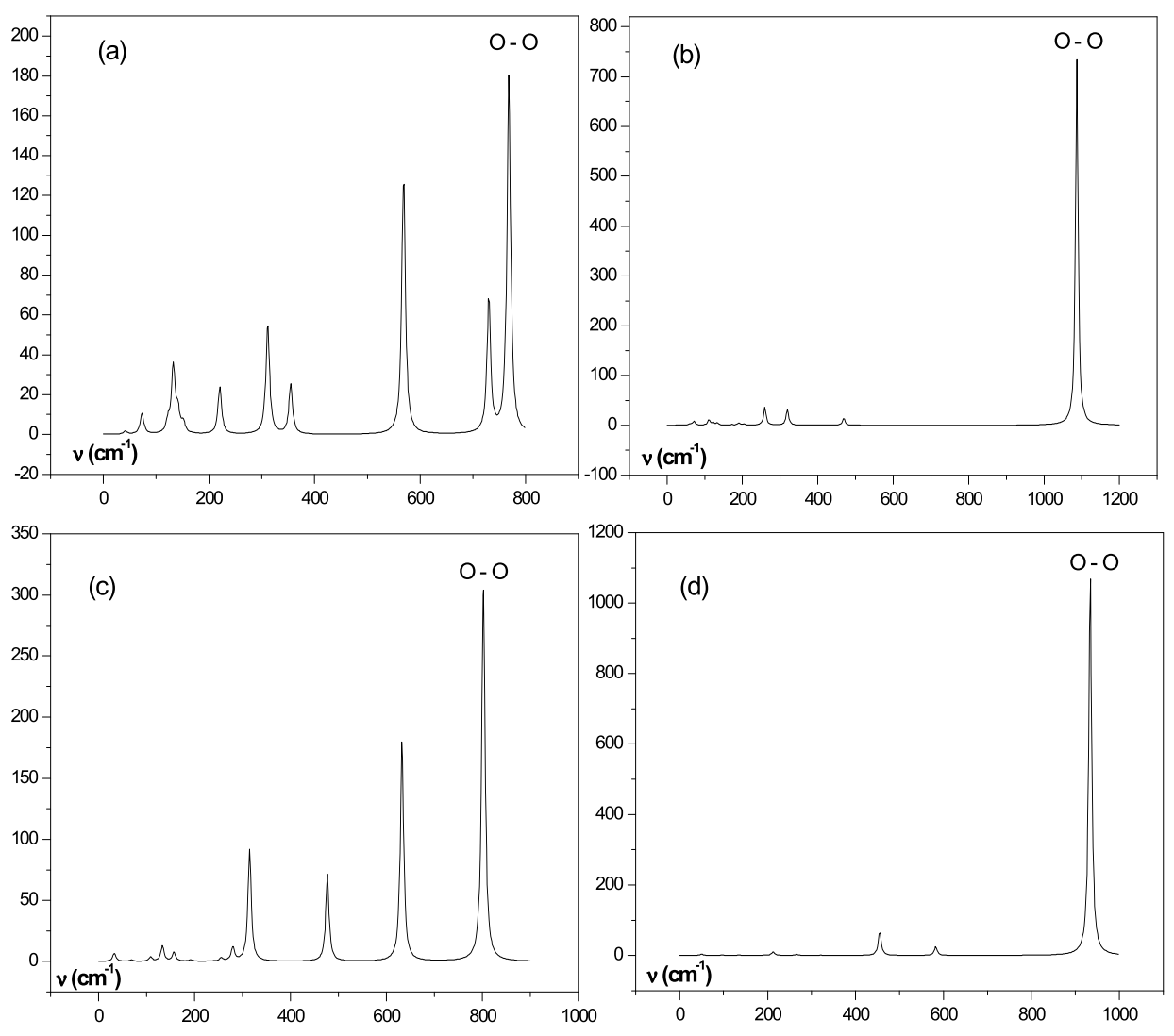

Figura 16: Espectros Teóricos de Infravermelho para as estruturas (a) $\mathrm{Pt}_{4}\left(\mathrm{O}_{2}\right)_{\text {ads }}$, (b) $\mathrm{Pt}_{3} \mathrm{Al}\left(\mathrm{O}_{2}\right)_{\text {ads, }}$ (c) $\mathrm{Pt}_{3} \mathrm{Cr}\left(\mathrm{O}_{2}\right)_{\text {ads }}$ e (d) $\mathrm{Pt}_{3} \mathrm{~V}\left(\mathrm{O}_{2}\right)_{\text {ads. }}$ [B3LYP/LANL2DZ, 6-311G**].

Enquanto isso, para estrutura $\mathrm{Pt}_{3} \mathrm{Cr}\left(\mathrm{O}_{2}\right)_{\text {ads }} \mathrm{o}$ estado fundamental é septeto e observamos que a adsorção de oxigênio molecular sobre o cluster $\mathrm{Pt}_{3} \mathrm{Cr}$, não é acompanhada de dissociação da molécula de $\mathrm{O}_{2}$. A adsorção ocorre em apenas seguindo o modelo de Ponte, onde um átomo de oxigênio está ligado a Pt e o outro ligado ao Cr. As distâncias interatômicas encontradas para as ligações Pt2 - O6 e Cr4 - O5 foram 2,158 e 2,066 Å, respectivamente. Enquanto isso, o comprimento da ligação $O$ - O obtido foi $1,327 \AA$, com frequência vibracional $1087,11 \mathrm{~cm}^{-1}$ e com cargas de Mulliken igual a -0,281 para o átomo $\mathrm{O5}$ (ligado a $\mathrm{Cr}$ ) e -0,169 para o átomo $\mathrm{O} 6$ (ligado a Pt). Esses dados podem caracterizar o estado superóxido para o oxigênio adsorvido na estrutura $\mathrm{Pt}_{3} \mathrm{Cr}\left(\mathrm{O}_{2}\right)_{\text {ads }}$ como intermediário da reação de redução de oxigênio. Observamos, também, um aumento no comprimento da 
ligação Pt3—Cr4 de 0,045 A após a interação com oxigênio molecular. Por fim, Os cálculos realizados para a estrutura $\mathrm{Pt}_{3} \mathrm{~V}\left(\mathrm{O}_{2}\right)_{\text {ads }}$ mostraram que o estado fundamental é quarteto, e a adsorção de oxigênio molecular também foi acompanhada de dissociação da ligação $O$ - $O$, com os átomos de oxigênio ligados assimetricamente aos átomos de $\mathrm{Pt}$ e $\mathrm{V}$, onde foi observado os valores para a distância Pt—O igual a 2,06 $\AA$ e ângulo O-Pt-V(5-2-4) de 73,537 enquanto que a distância $\mathrm{V}-\mathrm{O}$ encontrada foi de 1,808 A com ângulo O-V-Pt(6-4-2) igual a 76,926 . A distância interatômica $\mathrm{O}-\mathrm{O}$ passa a ser de $1,430 \AA$, com freqüência vibracional de $801,97 \mathrm{~cm}^{-1}$ e carga de Mulliken igual a -0,353 para o átomo de oxigênio ligado ao V e -0,311 para o átomo de oxigênio ligado a Pt. Esses dados podem caracterizar o estado peróxido para o oxigênio adsorvido na estrutura $\mathrm{Pt}_{3} \mathrm{~V}\left(\mathrm{O}_{2}\right)_{\text {ads }}$ como intermediário da reação de redução de oxigênio. Além disso, foi observado, também, uma pequena mudança no comprimento de ligação Pt2-V4, onde o comprimento de ligação sofre um diminuição de $0,154 \AA$.

Tabela 12: Distâncias interatômicas, multiplicidade de spin, cargas atômicas de Mulliken e freqüências de estiramento para $\mathrm{Pt}_{4}\left(\mathrm{O}_{2}\right)_{\text {ads }}, \operatorname{Pt} 3 \mathrm{Al}\left(\mathrm{O}_{2}\right)_{\text {ads }}, \operatorname{Pt}{ }_{3} \operatorname{Cr}\left(\mathrm{O}_{2}\right)_{\text {ads }}$ e $\mathrm{Pt}_{3} \mathrm{~V}\left(\mathrm{O}_{2}\right)_{\text {ads }}$.

\begin{tabular}{|c|c|c|c|c|c|}
\hline Estrutura & m & Distâncias (A) & Cargas (e) & Ângulo $\left({ }^{\circ}\right)$ & vo_-o $\left(\mathrm{cm}^{-1}\right)$ \\
\hline$\overline{\mathrm{Pt}}$ & 1 & $\begin{array}{l}1-2=2.719 \\
1-3=2.678 \\
1-4=2.525 \\
2-3=2.461 \\
2-4=2.554 \\
3-5=2.041 \\
2-6=1.997 \\
5-6=1.355\end{array}$ & $\begin{array}{l}\mathrm{Pt}_{1}=-0,051 \\
\mathrm{Pt}_{2}=+0,068 \\
\mathrm{Pt}_{3}=+0,355 \\
\mathrm{Pt}_{4}=+0,124 \\
\mathrm{O}_{5}=-0,242 \\
\mathrm{O}_{6}=-0,255\end{array}$ & $\begin{array}{l}1-2-3=62.010 \\
1-3-2=63.724 \\
2-1-3=54.265 \\
1-2-4=57.125 \\
1-4-2=64.728 \\
2-1-4=58.145 \\
3-5-6=103.71 \\
5-6-2=107.71 \\
3-2-6=73.860 \\
2-3-5=74.087\end{array}$ & 933,66 \\
\hline$\left(\mathrm{O}_{3}\right)_{3 d}$ & 2 & $\begin{array}{l}1-2=2.628 \\
1-4=2.324 \\
3-4=2.521 \\
2-3=2.646 \\
2-4=2.492 \\
3-6=1.966 \\
4-5=1.798 \\
5-6=1.477\end{array}$ & $\begin{array}{l}\mathrm{Pt}_{1}=-0,324 \\
\mathrm{Pt}_{2}=-0,178 \\
\mathrm{Pt}_{3}=-0,129 \\
\mathrm{Al}_{4}=+1,433 \\
\mathrm{O}_{5}=-0,491 \\
\mathrm{O}_{6}=-0,311\end{array}$ & $\begin{array}{l}1-2-3=103.217 \\
1-2-4=53.902 \\
1-4-2=66.046 \\
2-1-4=60.051 \\
4-2-3=58.661 \\
4-3-2=57.606 \\
2-4-3=63.732 \\
3-4-5=79.828 \\
4-3-6=67.143\end{array}$ & 768,19 \\
\hline
\end{tabular}




\begin{tabular}{|c|c|c|c|c|c|}
\hline $\mathrm{Pt}_{3} \mathrm{Cr}\left(\mathrm{O}_{2}\right)_{\text {ads }}$ & 7 & $\begin{array}{l}1-3=2.576 \\
1-4=2.706 \\
2-3=2.680 \\
2-4=2.444 \\
2-6=2.158 \\
4-5=2.066 \\
3-4=2.709 \\
5-6=1.327\end{array}$ & $\begin{array}{l}\mathrm{Pt}_{1}=-0,184 \\
\mathrm{Pt}_{2}=-0,112 \\
\mathrm{Pt}_{3}=-0,103 \\
\mathrm{Cr}_{4}=+0,849 \\
\mathrm{O}_{5}=-0,281 \\
\mathrm{O}_{6}=-0,169\end{array}$ & $\begin{array}{c}1-3-4=61.538 \\
1-4-3=56.814 \\
3-1-4=61.647 \\
2-3-4=53.932 \\
2-4-3=62.429 \\
3-2-4=63.637 \\
2-4-5=76.869 \\
4-5-6=105.103 \\
5-6-2=105.456 \\
6-2-4=72.510 \\
1-2-4=63.903 \\
2-1-4=54.195 \\
1-4-2=61.900 \\
1-2-3=57.707 \\
1-3-2=60.720 \\
2-1-3=61.572\end{array}$ & 1087,11 \\
\hline $\mathrm{Pt}_{3} \mathrm{~V}\left(\mathrm{O}_{2}\right)_{\mathrm{ads}}$ & 4 & $\begin{array}{l}1-3=2.571 \\
1-2=2.726 \\
1-4=2.589 \\
2-3=2.693 \\
2-4=2.407 \\
3-4=2.579 \\
2-5=2.061 \\
4-6=1.808 \\
5-6=1.430\end{array}$ & $\begin{array}{l}\mathrm{Pt}_{1}=-0,130 \\
\mathrm{Pt}_{2}=-0,026 \\
\mathrm{Pt}_{3}=-0,133 \\
\mathrm{~V}_{4}=+0,953 \\
\mathrm{O}_{5}=-0,353 \\
\mathrm{O}_{6}=-0,311\end{array}$ & $\begin{array}{l}1-3-4=60.376 \\
1-4-3=59.658 \\
3-1-4=59.965 \\
2-3-4=54.276 \\
2-4-3=65.275 \\
3-2-4=60.448 \\
2-4-6=76.926 \\
4-2-5=73.537 \\
1-2-4=60.213 \\
2-1-4=53.767 \\
1-4-2=66.019 \\
1-2-3=56.638 \\
1-3-2=62.335 \\
2-1-3=61.025\end{array}$ & 801,97 \\
\hline
\end{tabular}

A Tabela 13 apresenta as cargas atômicas e resultados das análises populacionais de Mulliken para as estruturas $\mathrm{Pt}_{4}\left(\mathrm{O}_{2}\right)_{\text {ads }}, \mathrm{Pt}_{3} \mathrm{Al}\left(\mathrm{O}_{2}\right)_{\text {ads }}, \mathrm{Pt}_{3} \mathrm{Cr}\left(\mathrm{O}_{2}\right)_{\text {ads }}$ e $\mathrm{Pt}_{3} \mathrm{~V}\left(\mathrm{O}_{2}\right)_{\text {ads }}$, onde estão contidas as populações de Mulliken das estruturas antes da interação para efeitos de comparação. As cargas atômicas de Mulliken mostram novamente um fluxo de carga no sentido dos clusters para os átomos de oxigênio. Nos resultados obtidos para $\mathrm{Pt}_{4}\left(\mathrm{O}_{2}\right)_{\text {ads }}$ as populações dos orbitais $\mathrm{s}$ e $\mathrm{p}$ dos átomos Pt2 e dos orbitais p do átomo Pt3 são maiores após a interação com oxigênio. Enquanto isso, as populações dos orbitais d do átomo Pt2 e dos orbitais s e d do átomo Pt3 diminuem após a adsorção. Além disso, houve um aumento nas populações dos orbitais $s$ do átomo Pt1 e diminuições nas populações dos orbitais d do átomo Pt1 e dos orbitais $\mathrm{p}$ e d do átomo Pt4. Por outro lado, as populações dos orbitais $p$ dos átomos de oxigênio aumentam de 4,04 para 4,28 após a interação. As 
análises de NBO revelaram a existência de múltiplas ligações e podemos destacar a ligações entre Pt1-Pt3, onde foi observada uma grande diferença de densidade, com contribuição exclusiva de Pt1, hibridizado $[\mathbf{s}(29 \%)+d(70 \%)]$, formando ligações $\sigma$ entre os átomos adjacentes. Comportamento parecido ocorre com a ligação Pt2-Pt3, onde o átomo Pt2 contribui de maneira mais efetiva para a ligação, apresentando maior participação de seus orbitais d (72\%), enquanto que o átomo Pt3 participa mais efetivamente com seus orbitais s (53\%). A explicação para que o átomo Pt3 contribua pouco para as ligações é o fato de que este átomo encontra-se diretamente ligado ao átomo de oxigênio que é mais eletronegativo que Pt. Isto fica mais evidente através da análise da ligação Pt3-O5, cuja caracterização é de 15\% de Pt $[s(10 \%)+p(45 \%)+d(45 \%)]$ e $85 \%$ de $O[s(6 \%)+p(94 \%)]$, a maior contribuição do oxigênio evidencia a deficiência de cargas sobre o átomo de $\mathrm{Pt}$ adjacente, cujas interações levam à formação de ligações do tipo $\sigma$. Foi observado, também, que a ligação Pt2-06 é idêntica a ligação Pt3-05. Ainda para esta estrutura temos três ligações 05-06 de dois centros que são formadas com simetria de densidade e participação expressiva dos orbitais $p$ destes átomos, formando ligações $\pi$. A transferência de carga dos orbitais $d$ da platina para os orbitais $p$ do oxigênio não é suficiente para provocar a quebra da ligação $O$ - O. Entretanto, essa transferência provoca um alongamento e enfraquecimento da ligação $O$ - $O$, quando comparado com a ligação $\mathrm{O}=\mathrm{O}$ do oxigênio molecular.

Nos resultados para $\mathrm{Pt}_{3} \mathrm{Al}\left(\mathrm{O}_{2}\right)_{\text {ads }}$ observamos que as populações dos orbitais d do átomo Pt3 sofrem uma diminuição considerável enquanto que o orbital s sofre aumento nas populações. Enquanto isso, o átomo Pt1 sofre uma pequena diminuição na população dos orbitais $p$ e pequeno aumento na população dos orbitais s e d. Além disso, para o átomo Al4 foi observado que as populações dos 
orbitais s diminuem em cerca de $60 \%$ enquanto que as populações do orbital $p$ sofrem acréscimos nas populações de Mulliken após interação com $\mathrm{O}_{2}$. Paralelamente a isso, os orbitais $p$ dos dois átomos de oxigênio sofrem aumento nas populações, principalmente o átomo 05 ligado à $A l 4$, resultando assim no preenchimento dos orbitais antiligantes $\left(\pi_{\mathrm{g}}{ }^{*}\right)$ da molécula de oxigênio, causando a quebra da ligação $\mathrm{O}-\mathrm{O}$ e formando as ligações $\mathrm{Pt3}-\mathrm{O} 6$ e Al4-O5. Análises de NBO revelaram que na Pt1-Pt2 temos uma participação bem mais evidente dos átomos de Pt1 (71\%) hibridizado com $\left(\mathrm{s}^{38 \%}+\mathrm{d}^{62 \%}\right)$, enquanto Pt2 contribui com $28 \%$ para a ligação, estando hibridizado com $\left(s^{92 \%}+p^{3 \%}+d^{5 \%}\right)$, formando uma ligação tipo $\sigma$. Em Pt1-Al4 a participação maior é da $\mathrm{Pt}(62 \%)$, que participa com $59 \%$ de seus orbitais s, formando ligações do tipo $\sigma$ com o $\mathrm{Al}[\mathrm{s}(44 \%)+p(55 \%)]$. O átomo Pt3 também liga-se ao átomo de $\mathrm{Al}$, e observa-se a mesma tendência de maior participação dos átomos de $\mathrm{Pt}$ (que participam com $69 \%$ de seus orbitais s), formando, também, ligações do tipo $\sigma$. Entre Pt e oxigênio forma-se a ligação Pt3-O6 com participação de $69 \%$ do átomo de $\mathrm{O}$ e $31 \%$ do átomo de $\mathrm{Pt} 3$, formando ligações com $71 \%$ de orbitais d da Pt e $92 \%$ dos orbitais $p$ do 0 . Ainda no spin $\alpha$ temos uma ligação Al4-O5 com participação mínima do átomo de $\mathrm{Al}(10 \%)$ hibridizado $[s(38 \%)+p(62 \%)]$ com o oxigênio hibridizado em $[s(25 \%)+p(75 \%)]$. A ligação O-O é caracterizada pela simetria de overlap e participação de aproximadamente $88 \%$ dos orbitais p na ligação entre estes átomos. No spin beta as ligações também são múltiplas. Dentre as mais importantes temos: Pt3-O6 com participação maior do oxigênio com seus orbitais p presentes em $94 \%$ para a formação das ligações, que se sobrepõem aos orbitais $\mathrm{d}(73 \%)$ da $\mathrm{Pt}$. $\mathrm{O}$ átomo $\mathrm{O5}$ liga-se ao Al4 formando ligações $\sigma$ com pequena contribuição do átomo de $\mathrm{Al}$, assim como foi observado no spin alfa. As contribuições calculadas foram de $12 \%$ de $\mathrm{Al}$ 
$[s(66 \%)+p(34 \%)]$ e $88 \%$ de $O[s(24 \%)+p(76 \%)]$, esta é uma ligação do tipo $\sigma$ hibridizada. Em O-O temos a mesma simetria de overlap e mesma intensidade de orbitais $p$ de ambos os átomos participantes da ligação.

Nos resultados para $\operatorname{Pt} t_{3} \mathrm{Cr}\left(\mathrm{O}_{2}\right)_{\text {ads }}$ observamos que as populações dos orbitais $\mathrm{d}$ de todos os átomos Pt sofrem uma diminuições enquanto que os orbitais $p$ do átomo Pt2 sobrem um pequeno aumento nas populações. Por outro lado, as populações dos orbitais $\mathrm{p}$ e d do átomo $\mathrm{Cr}$ diminuem após a interação com oxigênio molecular. Além disso, observamos acréscimos nas populações dos orbitais $p$ dos átomos de oxigênio como resultado desta interação. Para compreender melhor as ligações existentes os resultados de NBO revelaram a existência de duas ligações no spin alfa onde a primeira ligação é entre Pt1 - Pt3 formada com maior contribuição de $\mathrm{Pt} 1$ (56\%) com envolvendo $91 \%$ dos orbitais s e o átomo $\mathrm{Pt} 3$ contribui com $44 \%$ também envolvendo $91 \%$ dos orbitais s, formando uma ligação $\sigma_{\mathrm{s}-s} ;$ a segunda ligação encontrada foi entre O-O, resultante da contribuição de $50 \%$ de cada átomo e envolvendo cerca de $86 \%$ dos orbitais $p[s(14 \%)+p(86 \%)]$ o que pode caracterizar uma ligação $\pi$. No spin beta, encontramos múltiplas ligações: a primeira observada foi a ligação entre Pt1-Pt2 formada pela contribuição de $60 \%$ $\operatorname{Pt} 1[s(58 \%)+d(41 \%)]$ e $40 \%$ de Pt2 $[s(27 \%)+p(9 \%)+d(64 \%)]$, formando uma ligação $\sigma$ hibridizada. A segunda ligação foi entre Pt1-Pt3, também foi uma ligação hibridizada, formada com contribuição de $52 \%$ de Pt1 [s(35\%) + p(64\%)] e 48\% Pt3 $[s(45 \%)+d(54 \%)]$. A ligação entre Pt2-Pt3 encontrada foi semelhante à ligação entre Pt1-Pt2. Outra ligação encontrada foi $\sigma$ para Pt1-Cr4, resultante da ligação entre os orbitais híbridos com contribuições majoritárias de Pt1 (89\%) [s(30\%) + $d(68 \%)]$ e $11 \%$ de $\mathrm{Cr} 4[\mathrm{~s}(17 \%)+p(6 \%)+d(77 \%)]$. Por fim, foram observadas duas ligações O5-O6, ambas com contribuições de $50 \%$ de cada átomo, onde a primeira 
envolve uma hibridização entre os orbitais $s^{16 \%}$ e $p^{85 \%}$, enquanto que a segunda envolve basicamente os orbitais $p(99,88 \%)$.

Tabela 13: Cargas atômicas e resultados das análises populacionais de Mulliken para as estruturas $\mathrm{Pt}_{4}, \mathrm{Pt}_{3} \mathrm{Al}, \mathrm{Pt}_{3} \mathrm{Cr}, \mathrm{Pt}_{3} \mathrm{~V}$ [B3LYP/LANL2DZ], $\mathrm{O}_{2}$ [B3LYP/6-311G* ], $\mathrm{Pt}_{4}\left(\mathrm{O}_{2}\right)_{\text {ads }}, \mathrm{Pt}_{3} \mathrm{Al}\left(\mathrm{O}_{2}\right)_{\text {ads }}, \mathrm{Pt}_{3} \mathrm{Cr}\left(\mathrm{O}_{2}\right)_{\text {ads }}$ e $\mathrm{Pt}_{3} \mathrm{~V}\left(\mathrm{O}_{2}\right)_{\text {ads }}$ [B3LYP/LANL2DZ, 6-311G**].

\begin{tabular}{|c|c|c|c|c|c|c|c|}
\hline Estrutura & & $\mathrm{Pt}(1)$ & $\mathrm{Pt}(2)$ & $\mathrm{Pt}(3)$ & $\mathrm{Pt}(4)$ & $\mathrm{O}(5)$ & $\mathrm{O}(6)$ \\
\hline \multirow[t]{4}{*}{$\mathrm{Pt}_{4}$} & Carga & $+0,004$ & $+0,004$ & $-0,004$ & $-0,004$ & - & - \\
\hline & $S$ & 2,70 & 2,70 & 2,72 & 2,72 & - & - \\
\hline & $P$ & 6,14 & 6,14 & 6,17 & 6,17 & - & - \\
\hline & $D$ & 9,16 & 9,16 & 9,10 & 9,10 & - & - \\
\hline \multirow[t]{4}{*}{$\mathrm{O}_{2}$} & Carga & - & - & & & 0 & 0 \\
\hline & $S$ & - & - & & & 3,93 & 3,93 \\
\hline & $P$ & - & - & & & 4,04 & 4,04 \\
\hline & $D$ & - & - & & & 0,03 & 0,03 \\
\hline \multirow[t]{4}{*}{$\mathrm{Pt}_{4}\left(\mathrm{O}_{2}\right)_{\mathrm{ads}}$} & Carga & $-0,051$ & $+0,068$ & $+0,355$ & $+0,124$ & $-0,242$ & $-0,255$ \\
\hline & $S$ & 2,82 & 2,79 & 2,56 & 2,69 & 3,95 & 3,95 \\
\hline & $P$ & 6,16 & 6,42 & 6,21 & 6,06 & 4,27 & 4,28 \\
\hline & $D$ & 9,06 & 8,72 & 8,88 & 9,12 & 0,02 & 0,02 \\
\hline Estrutura & & $\mathrm{Pt}(1)$ & $\mathrm{Pt}(2)$ & $\mathrm{Pt}(3)$ & $\mathrm{Al}(4)$ & $\mathrm{O}(5)$ & $\mathrm{O}(6)$ \\
\hline \multirow[t]{4}{*}{$\mathrm{Pt}_{3} \mathrm{Al}$} & Carga & $-0,231$ & $-0,231$ & $-0,231$ & $+0,694$ & - & - \\
\hline & $\mathrm{S}$ & 2,85 & 2,85 & 2,85 & 1,63 & - & - \\
\hline & $P$ & 6,22 & 6,22 & 6,22 & 0,69 & - & - \\
\hline & $D$ & 9,16 & 9,16 & 9,16 & 0,00 & - & - \\
\hline \multirow{4}{*}{$\mathrm{O}_{2}$} & Carga & - & - & & & 0 & 0 \\
\hline & $S$ & - & - & & & 3,93 & 3,93 \\
\hline & $P$ & - & - & & & 4,04 & 4,04 \\
\hline & $\mathrm{D}$ & - & - & & & 0,03 & 0,03 \\
\hline \multirow[t]{4}{*}{$\mathrm{Pt}_{3} \mathrm{Al}\left(\mathrm{O}_{2}\right)_{\text {ad: }}$} & Carga & $-0,324$ & $-0,178$ & $-0,129$ & $+1,433$ & $-0,491$ & $-0,311$ \\
\hline & $s$ & 2,91 & 2,83 & 3,02 & 0,61 & 3,93 & 3,97 \\
\hline & $P$ & 6,11 & 6,21 & 6,25 & 0,95 & 4,54 & 4,33 \\
\hline & D & 9,30 & 9,14 & 8,85 & 0,00 & 0,01 & 0,01 \\
\hline Estrutura & & $\mathrm{Pt}(1)$ & $\mathrm{Pt}(2)$ & $\operatorname{Pt}(3)$ & $\mathrm{Cr}(4)$ & $\mathrm{O}(5)$ & $\mathrm{O}(6)$ \\
\hline \multirow[t]{4}{*}{$\mathrm{Pt}_{3} \mathrm{Cr}$} & Carga & $-0,223$ & $-0,223$ & $-0,223$ & $+0,669$ & - & - \\
\hline & $\mathrm{S}$ & 2,87 & 2,87 & 2,87 & 2,30 & - & - \\
\hline & $P$ & 6,14 & 6,14 & 6,14 & 6,67 & - & - \\
\hline & $D$ & 9,21 & 9,21 & 9,21 & 4,93 & - & - \\
\hline \multirow[t]{4}{*}{$\mathrm{O}_{2}$} & Carga & - & - & & & 0 & 0 \\
\hline & S & - & - & & & 3,93 & 3,93 \\
\hline & $P$ & - & - & & & 4,04 & 4,04 \\
\hline & D & - & - & & & 0,03 & 0,03 \\
\hline \multirow{4}{*}{$\mathrm{Pt}_{3} \mathrm{Cr}\left(\mathrm{O}_{2}\right)_{\mathrm{ad}}$} & Carga & $-0,184$ & $-0,112$ & $-0,103$ & $+0,849$ & $-0,281$ & $-0,169$ \\
\hline & S & 2,92 & 2,86 & 2,83 & 2,28 & 3,92 & 3,93 \\
\hline & $\mathrm{P}$ & 6,07 & 6,29 & 6,12 & 6,17 & 4,34 & 4,21 \\
\hline & $D$ & 9,18 & 8,96 & 9,15 & 4,69 & 0,01 & 0,01 \\
\hline Estrutura & & $\mathrm{Pt}(1)$ & $\mathrm{Pt}(2)$ & $\mathrm{Pt}(3)$ & $V(4)$ & $\mathrm{O}(5)$ & $\mathrm{O}(6)$ \\
\hline \multirow[t]{4}{*}{$\mathrm{Pt}_{3} \mathrm{~V}$} & Carga & $-0,235$ & $-0,235$ & $-0,305$ & 0,776 & - & - \\
\hline & $\mathrm{S}$ & 2,94 & 2,94 & 3,17 & 2,35 & - & - \\
\hline & $P$ & 6,16 & 6,16 & 6,15 & 6,18 & - & - \\
\hline & D & 9,13 & 9,13 & 8,98 & 3,68 & - & - \\
\hline \multirow[t]{4}{*}{$\mathrm{O}_{2}$} & Carga & - & - & & & 0 & 0 \\
\hline & S & - & - & & & 3,93 & 3,93 \\
\hline & $\mathrm{P}$ & - & - & & & 4,04 & 4,04 \\
\hline & D & - & - & & & 0,03 & 0,03 \\
\hline \multirow[t]{4}{*}{$\mathrm{Pt}_{3} \mathrm{~V}\left(\mathrm{O}_{2}\right)_{\mathrm{ads}}$} & Carga & $-0,130$ & $-0,026$ & $-0,133$ & $+0,953$ & $-0,353$ & $-0,311$ \\
\hline & $\mathrm{S}$ & 2,94 & 2,87 & 2,94 & 2,29 & 3,96 & 3,92 \\
\hline & $\mathrm{P}$ & 6,07 & 6,32 & 6,09 & 6,17 & 4,33 & 4,41 \\
\hline & $D$ & 9,12 & 8,83 & 9,13 & 3,59 & 0,01 & 0,01 \\
\hline
\end{tabular}


Nos resultados para $\mathrm{Pt}_{3} \mathrm{~V}\left(\mathrm{O}_{2}\right)_{\text {ads }}$ observamos que as populações dos orbitais $\mathrm{s}$ dos átomos Pt2, Pt3 diminuem enquanto que há um aumento na população dos orbitais d de Pt3 e diminuição nos orbitais d de Pt2, pois parte dessa carga é transferida para o orbital p de 05. Além disso, para o átomo V4 foi observado que as populações dos orbitais $\mathrm{s}$ e d diminuem após interação com $\mathrm{O}_{2}$, ocorrendo transferência de carga para os orbitais $p$ do 06 , resultando, assim, no preenchimento dos orbitais antiligantes $\left(\pi_{\mathrm{g}}{ }^{*}\right)$ da molécula de oxigênio, causando a quebra da ligação O—O e formando as ligações Pt2—O5 e V4-O6. Análises de NBO mostraram que no spin $\alpha \mathrm{Pt} 2$ se liga a $\mathrm{O} 5$ em que o átomo $\mathrm{Pt}$ contribui minoritariamente $(24 \%)$ formando um uma $\sigma$ hibridizada $[s(43 \%)+d(56 \%)]$ com oxigênio que contribui com $76 \%[s(9 \%)+p(91 \%)]$. Observamos, também, a existência de duas ligações entre V4 - O6 onde a primeira ligação é formada com contribuição de $10 \%$ de Vanádio $[\mathrm{p}(47 \%)+\mathrm{d}(53 \%)$ e oxigênio contribuindo com $90 \%$ de seus orbitais p $(99,8 \%)$. A segunda ligação $V$ - O é uma ligação formada com $21 \%$ de contribuição de vanádio envolvendo $91 \%$ dos seus orbitais d e oxigênio contribuindo com $89 \%$ na ligação com orbitais hibridizados $[\mathbf{s}(10 \%)+p(90 \%)]$. Além disso, observamos uma ligação O - O com $50 \%$ de contribuição de cada átomo envolvendo os orbitais p em cerca de $90 \%$. No spin $\beta$ encontramos uma ligação V O com contribuição de $10 \%$ de vanádio $[s(8 \%)+p(12 \%)+d(80 \%)]$ e $90 \%$ de oxigênio $[p(99 \%)]$. Por fim, analisamos uma também uma ligação O - O em que o átomo 05 contribui com $46 \%[s(10 \%)+p(89 \%)]$ e o átomo $06[s(13 \%)+p(87 \%)]$.

Para obtermos as barreiras de energias de dissociação da ligação $\mathrm{O}-\mathrm{O}$ após a interação com clusters $\mathrm{Pt}_{3} \mathrm{Al}$ e $\mathrm{Pt}_{3} \mathrm{~V}$, curvas de superfície de energia potencial foram calculadas e são apresentadas na Figura 17. Como pode ser observado, foi encontrado o valor de aproximadamente $0,46 \mathrm{eV}$ para a barreira de dissociação da 
ligação $\mathrm{O}-\mathrm{O}$ adsorvido sobre o cluster $\mathrm{Pt}_{3} \mathrm{Al}$ e aproximadamente $0,28 \mathrm{eV}$ para a barreira energética de dissociação da ligação $\mathrm{O}-\mathrm{O}$ adsorvido sobre o cluster $\mathrm{Pt}_{3} \mathrm{~V}$. Esses resultados mostram que a dissociação de $\mathrm{O}_{2}$ ocorre mais facilmente sobre o cluster $\mathrm{Pt}_{3} \mathrm{~V}$ que sobre o cluster $\mathrm{Pt}_{3} \mathrm{Al}$.

Os resultados encontrados para as energias de ligação entre oxigênio molecular e os clusters $\mathrm{Pt} 4, \mathrm{Pt}_{3} \mathrm{Al}, \mathrm{Pt}_{3} \mathrm{Cr}$ e $\mathrm{Pt}_{3} \mathrm{~V}$ são apresentados na Tabela 14, onde também estão inseridos os dados termodinâmicos, tais como variação de entalpia, variação de energia livre e variação de entropia a 298,15 K e 1 atm de pressão. Podemos observar novamente que a ligação torna-se mais forte quando um átomo de platina é substituído por outro metal. $\mathrm{O}$ valor da energia de ligação entre $\mathrm{Pt}_{4} \mathrm{e} \mathrm{O}_{2}$ foi de $-2,02 \mathrm{eV}$ e esse valor aumenta para $-3,00 \mathrm{eV},-2,88 \mathrm{eV}$ e $-3,98$ $\mathrm{eV}$ quando um átomo de platina é substituído por átomos de $\mathrm{Al}, \mathrm{Cr}$ e $\mathrm{V}$, respectivamente. Além disso, podemos observar que a adsorção de oxigênio sobre os clusters $\mathrm{Pt}_{2} \mathrm{Al}, \mathrm{Pt}_{2} \mathrm{Cr}$ e $\mathrm{Pt}_{2} \mathrm{~V}$ é uma reação muito exotérmica e envolve maior variação de entalpia e energia livre, que para a adsorção no cluster $\mathrm{Pt}_{3} \mathrm{~V}$. Dentre os clusters estudados o $\mathrm{Pt}_{3} \mathrm{~V}$ foi o que apresentou maior calor de adsorção e maior variação de energia livre. Além disso, todas as reações apresentaram variações negativas de entropia e não foram observadas diferenças significativas na variação de entropia para adsorção de oxigênio. 


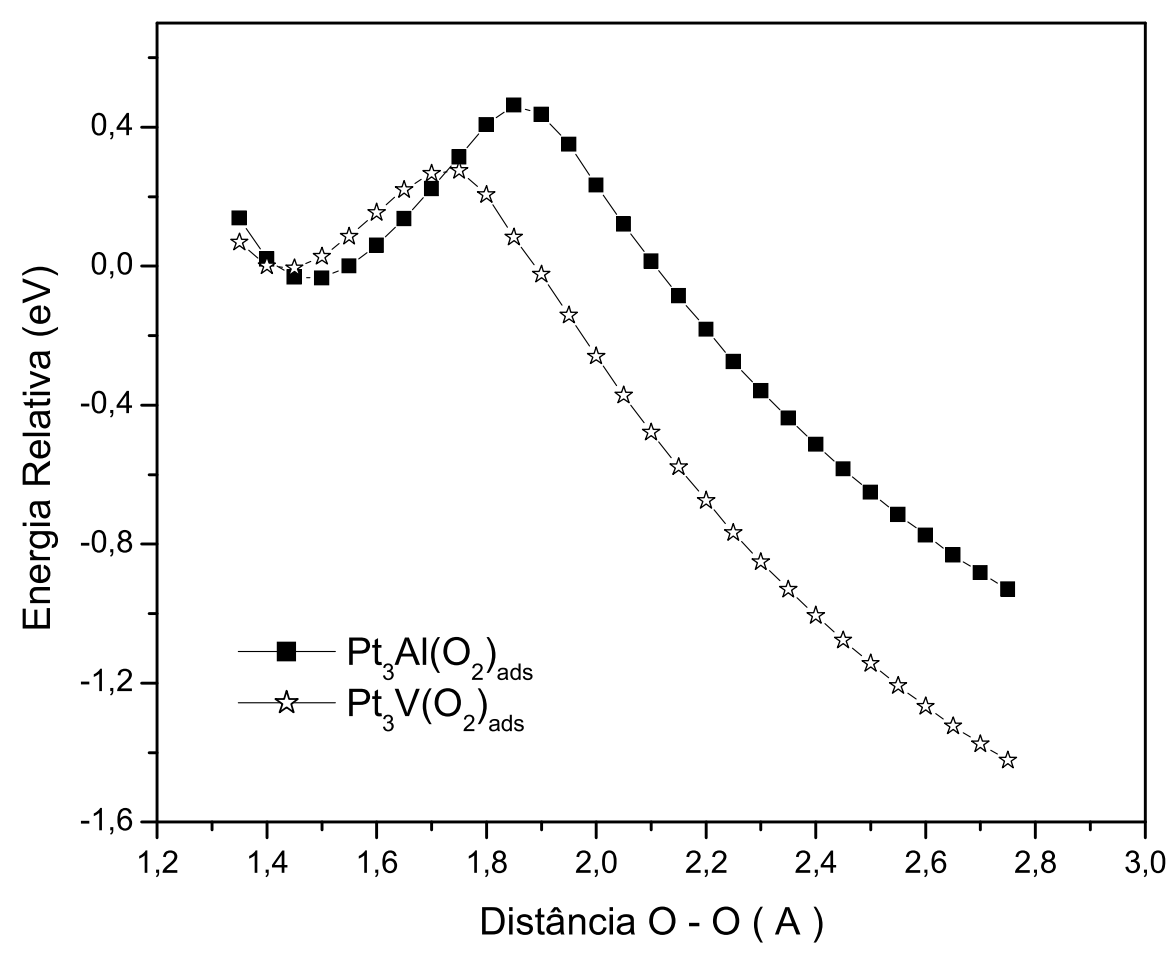

Figura 17: Curva de superfície de energia potencial com barreira energética para a dissociação de oxigênio molecular adsorvido em $\mathrm{Pt}_{3} \mathrm{Al}_{\mathrm{l}}$ e $\mathrm{Pt}_{3} \mathrm{~V}$ [B3LYP/LANL2DZ, 6$\left.311 \mathrm{G}^{\star \star}\right]$. Energias calculadas em relação às energias das estruturas otimizadas.

Tabela 14: Energia de ligação, variação de entalpia, variação de energia livre e variação de entropia para a reação: $\mathrm{Pt}_{3} \mathrm{M}+\mathrm{O}_{2} \rightarrow \mathrm{Pt}_{2} \mathrm{M}\left(\mathrm{O}_{2}\right)_{\text {ads }}$. Dados Termodinâmicos obtidos a 298,15 K e p=1 atm. [B3LYP/LANL2DZ, 6-311G**].

$\begin{array}{ccccc}\text { Reação } & \mathbf{E}_{\text {Ligação }}(\mathbf{e V}) & \boldsymbol{\Delta H}\left(\mathbf{k c a l ~ m o l} \mathbf{~}^{-1}\right) & \boldsymbol{\Delta} \mathbf{G}\left(\mathbf{k c a l ~ m o l}^{-1}\right) & \Delta \mathbf{S}\left(\mathbf{c a l ~ K}^{-1} \mathbf{m o l}^{-1}\right) \\ \mathrm{Pt}_{4}+\mathrm{O}_{2} \rightarrow \mathrm{Pt}_{4}\left(\mathrm{O}_{2}\right) \text { ads } & -2,02 & 47,37 & -35.64 & -39,37 \\ \mathrm{Pt}_{3} \mathrm{Al}+\mathrm{O}_{2} \rightarrow \mathrm{Pt}_{3} \mathrm{Al}\left(\mathrm{O}_{2}\right)_{\text {ads }} & -3,00 & -69,89 & -59,35 & -35,39 \\ \mathrm{Pt}_{3} \mathrm{Cr}+\mathrm{O}_{2} \rightarrow \mathrm{Pt}_{3} \mathrm{Cr}\left(\mathrm{O}_{2}\right)_{\text {ads }} & -2,88 & -66,90 & -56,32 & -35,51 \\ \mathrm{Pt}_{3} \mathrm{~V}+\mathrm{O}_{2} \rightarrow \mathrm{Pt}_{3} \mathrm{~V}\left(\mathrm{O}_{2}\right)_{\text {ads }} & -3,98 & -92,57 & -81,02 & -38,73\end{array}$




\subsection{Interação de Oxigênio Molecular com Clusters $\mathrm{Pt}_{5}, \mathrm{Pt}_{4} \mathrm{Al}_{1} \mathrm{Pt}_{4} \mathrm{Cr}, \mathrm{Pt}_{4} \mathrm{~V}$.}

Na Figura 18 são apresentadas as estruturas otimizadas para a adsorção de oxigênio molecular sobre os clusters $\mathrm{Pt}_{5}, \mathrm{Pt}_{4} \mathrm{Al}, \mathrm{Pt}_{4} \mathrm{Cr}_{2}$ e $\mathrm{Pt}_{4} \mathrm{~V}$ e na Tabela 15 estão resumidos os valores de cargas atômicas de Mulliken, multiplicidades de spin, comprimentos de ligação, ângulos e freqüências de estiramento da ligação O—O, obtidas a partir dos espectros teóricos de infravermelho mostrados na Figura 19, das estruturas otimizadas $\mathrm{Pt}_{5}\left(\mathrm{O}_{2}\right)_{\text {ads }}, \quad \mathrm{Pt} 4 \mathrm{Al}\left(\mathrm{O}_{2}\right)_{\text {ads }}, \quad \mathrm{Pt} 4 \mathrm{Cr}\left(\mathrm{O}_{2}\right)_{\text {ads }}$ e $\mathrm{Pt}_{4} \mathrm{~V}\left(\mathrm{O}_{2}\right)_{\text {ads }}$. Os resultados obtidos mostraram que o estado fundamental para $\mathrm{Pt}_{5}\left(\mathrm{O}_{2}\right)_{\text {ads }}$ é singlete, onde ocorre a adsorção não dissociativa da molécula de oxigênio, com cada átomo de oxigênio ligado simetricamente aos átomos de platina com distância $\mathrm{Pt}-\mathrm{O}$ igual a $2,029 \AA$ e $O$ - O igual a $1,348 \AA$ com frequência de estiramento $903,52 \mathrm{~cm}^{-1} \mathrm{e}$ com cargas de Mulliken igual a -0,246 o que caracteriza, dessa forma, o estado superóxido para o oxigênio adsorvido, como intermediário da reação de redução de oxigênio em $\mathrm{Pt}_{5}$. Além disso, a ligação Pt2—Pt4 sofre relaxação após a adsorção de oxigênio e seu comprimento aumenta em 0,224 $\AA$. Para estrutura $\mathrm{Pt}_{4} \mathrm{Al}\left(\mathrm{O}_{2}\right)_{\text {ads }} \mathrm{O}$ estado fundamental é quarteto, e a adsorção de oxigênio molecular é acompanhada de dissociação da molécula de oxigênio. Entretanto, os átomos de oxigênio estão ligado assimetricamente aos átomos de Pt e Al, com distância Pt—O igual a 1,99 $\AA$ e ângulo O-Pt-Al(5-2-7) de 66,377 enquanto que a distância $\mathrm{Al}-\mathrm{O}$ encontrada foi de 1,919 A com ângulo O-Al-Pt(2-5-6) igual a 79,946 . A distância interatômica O—O passa a ser de 1,456 $\AA$, com freqüência vibracional de $796,92 \mathrm{~cm}^{-1}$ e com carga de Mulliken igual a -0,476 para o átomo de oxigênio ligado ao $\mathrm{Al}$ e $-0,302$ para o átomo de oxigênio ligado a Pt. Esses dados podem caracterizar o estado peróxido para o oxigênio adsorvido na estrutura $\mathrm{Pt}_{4} \mathrm{Al}\left(\mathrm{O}_{2}\right)_{\text {ads }}$ como intermediário da reação de redução de oxigênio. Observamos também uma pequena relaxação na ligação Pt— 
Al, após a interação com oxigênio molecular, onde o comprimento de ligação sofre um aumento de $0,012 \AA$. Enquanto isso, para estrutura $\operatorname{Pt} 4 \mathrm{Cr}\left(\mathrm{O}_{2}\right)_{\text {ads }} \circ$ estado fundamental é quinteto e observamos que a adsorção de oxigênio molecular sobre o cluster $\mathrm{Pt}_{4} \mathrm{Cr}$, não é acompanhada de dissociação da molécula de $\mathrm{O}_{2}$. A adsorção ocorre em apenas um sítio catalítico, seguindo o modelo de Pauling, que foi o átomo de platina, formando um ângulo de ligação Pt-O-O igual a 104,818 , distância de ligação Pt—O igual a 2,039 A. Esse valor está 0,089 A maior que o calculado para a interação entre átomo de platina e oxigênio molecular, onde a estrutura encontrada foi semelhante a do modelo Pauling, com distância Pt—O aproximadamente a 1,95 A. Além disso, após a adsorção o comprimento de ligação O—O aumentou em 0,11 $\AA$, quando comparado com o comprimento da ligação $\mathrm{O}_{2}$, a frequência vibracional da ligação foi $983,26 \mathrm{~cm}^{-1}$. A carga obtida para o átomo de oxigênio ligado ao átomo $\mathrm{Pt}$ foi $-0,233(\mathrm{O})$ e e -0,286 para o outro átomo de oxigênio $(\mathrm{O})$.

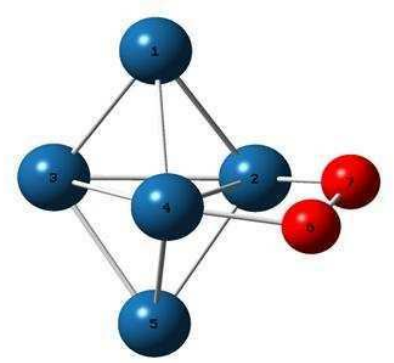

$\mathrm{Pt}_{5}\left(\mathrm{O}_{2}\right)_{\text {ads }}$

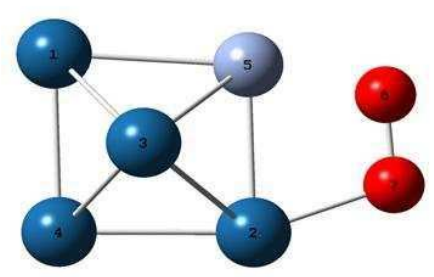

$\operatorname{Pt}{ }_{4} \mathrm{Cr}\left(\mathrm{O}_{2}\right)_{\text {ads }}$

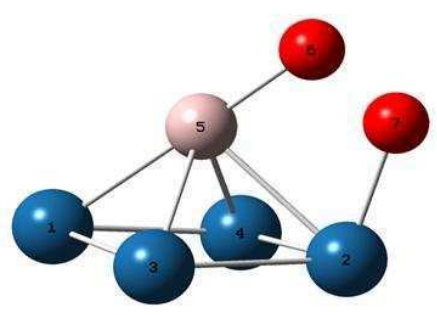

$\mathrm{Pt}_{4} \mathrm{Al}\left(\mathrm{O}_{2}\right)_{\text {ads }}$

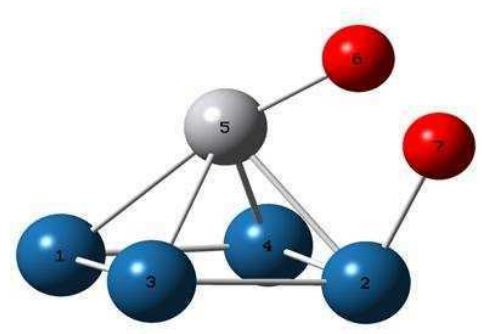

$\mathrm{Pt}_{4} \mathrm{~V}\left(\mathrm{O}_{2}\right)_{\text {ads }}$

Figura 18: Geometrias das estruturas (a) $\mathrm{Pt}_{5}\left(\mathrm{O}_{2}\right)_{\text {ads }}$, (b) $\mathrm{Pt} 4 \mathrm{Al}\left(\mathrm{O}_{2}\right)_{\text {ads }}$, (c) $\mathrm{Pt} 4 \mathrm{Cr}\left(\mathrm{O}_{2}\right)_{\text {ads }}$ e (d) $\mathrm{Pt}_{4} \mathrm{~V}\left(\mathrm{O}_{2}\right)_{\text {ads }}$ otimizadas com o método B3LYP/LANL2DZ, 6-311G**. 
Os cálculos realizados para a estrutura $\mathrm{Pt}_{4} \mathrm{~V}\left(\mathrm{O}_{2}\right)_{\text {ads }}$ mostraram que o estado fundamental é sexteto, e a adsorção de oxigênio molecular também foi acompanhada de dissociação da ligação $\mathrm{O}-\mathrm{O}$, onde os átomos de oxigênio estão ligado assimetricamente aos átomos de $\mathrm{Pt}$ e $\mathrm{V}$, onde foi observado a distância $\mathrm{Pt}$-O igual a 1,95 A e ângulo O-Pt-V(5-2-7) de $69,701^{\circ}$ enquanto que a distância $\mathrm{V}-\mathrm{O}$ encontrada foi de 1,86 A com ângulo O-V-Pt(2-5-6) igual a 77,176. A distância interatômica $O-O$ passa a ser de $1,433 \AA$, com freqüência vibracional de 814,34 $\mathrm{cm}^{-1}$ e com carga de Mulliken igual a -0,339 para o átomo de oxigênio ligado ao $\mathrm{V}$ e 0,284 para o átomo de oxigênio ligado a Pt. Esses dados podem caracterizar o estado peróxido para o oxigênio adsorvido na estrutura $\mathrm{Pt}_{4} \mathrm{~V}\left(\mathrm{O}_{2}\right)_{\text {ads }}$ como intermediário da reação de redução de oxigênio. Observamos também uma pequena relaxação na ligação $\mathrm{Pt}-\mathrm{V}$, após a interação com oxigênio molecular, onde o comprimento de ligação sofre um aumento de 0,061 $\AA$.

Tabela 15: Distâncias interatômicas, multiplicidade de spin, cargas atômicas de Mulliken e freqüências de estiramento para $\mathrm{Pt}_{5}\left(\mathrm{O}_{2}\right)_{\text {ads }}, \operatorname{Pt} 4 \mathrm{Al}\left(\mathrm{O}_{2}\right)_{\text {ads }}, \mathrm{Pt}_{4} \mathrm{Cr}\left(\mathrm{O}_{2}\right)_{\text {ads }}$ e $\mathrm{Pt}_{4} \mathrm{~V}\left(\mathrm{O}_{2}\right)$ ads.

\begin{tabular}{|c|c|c|c|c|c|c|}
\hline Cluster & $\mathbf{m}$ & Distâncias (A) & Cargas (e) & Ângulo ( $\left.{ }^{\circ}\right)$ & Ângulo ( ${ }^{\circ}$ ) & $v_{0 .-0}\left(\mathrm{~cm}^{-1}\right)$ \\
\hline $\mathrm{P}$ & 1 & $\begin{array}{l}1-2=2.722 \\
1-3=2.620 \\
1-4=2.642 \\
2-3=2.820 \\
2-4=2.843 \\
3-4=2.820 \\
2-5=2.642 \\
3-5=2.620 \\
4-5=2.722 \\
4-6=2.029 \\
2-7=2.029 \\
6-7=1.348\end{array}$ & $\begin{array}{l}\mathrm{Pt}_{1}=-0,061 \\
\mathrm{Pt}_{2}=+0,167 \\
\mathrm{Pt}_{3}=+0,036 \\
\mathrm{Pt}_{4}=+0,167 \\
\mathrm{Pt}_{5}=-0,061 \\
\mathrm{O}_{6}=-0,246 \\
\mathrm{O}_{7}=-0,246\end{array}$ & $\begin{array}{l}1-2-3=56.381 \\
1-3-2=59.920 \\
2-1-3=63.700 \\
2-3-4=60.545 \\
3-2-4=59.715 \\
2-4-3=59.739 \\
1-3-4=57.976 \\
1-4-3=57.218 \\
3-1-4=64.805 \\
1-2-4=56.628 \\
1-4-2=59.378 \\
2-1-4=63.994\end{array}$ & $\begin{array}{c}4-2-7=66.973 \\
2-7-6=108.690 \\
4-6-7=108.686 \\
2-4-6=66.976 \\
3-4-5=56.388 \\
4-3-5=59.931 \\
3-4-5=63.680 \\
4-2-5=59.378 \\
2-4-5=56.627 \\
2-5-4=63.993 \\
2-5-3=64.823 \\
3-5-4=63.680 \\
\end{array}$ & 903,52 \\
\hline & 4 & $\begin{array}{l}1-3=2.658 \\
1-4=2.658 \\
1-5=2.414 \\
2-3=2.690 \\
2-4=2.690 \\
2-5=2.570 \\
3-5=2.504 \\
4-5=2.504 \\
2-7=1.990 \\
5-6=1.816 \\
6-7=1.456\end{array}$ & $\begin{array}{l}\mathrm{Pt}_{1}=-0,241 \\
\mathrm{Pt}_{2}=-0,092 \\
\mathrm{Pt}_{3}=-0,153 \\
\mathrm{Pt}_{4}=-0,153 \\
\mathrm{Al}_{5}=+1,418 \\
\mathrm{O}_{6}=-0,476 \\
\mathrm{O}_{7}=-0,302\end{array}$ & $\begin{array}{l}1-3-2=91.923 \\
3-2-4=87.280 \\
2-4-1=91.922 \\
4-1-3=88.587 \\
1-3-5=55.654 \\
3-1-5=58.944 \\
1-5-3=65.401 \\
2-3-5=59.197 \\
3-2-5=56.807 \\
2-5-3=63.996 \\
2-4-5=59.197\end{array}$ & $\begin{array}{l}4-2-5=56.806 \\
2-5-4=63.995 \\
1-4-5=55.654 \\
4-1-5=58.943 \\
1-5-4=65.401 \\
2-5-6=79.946 \\
5-2-7=66.377\end{array}$ & 796,92 \\
\hline
\end{tabular}


Resultados e Discussões

\begin{tabular}{|c|c|c|c|c|c|c|}
\hline $\mathrm{Pt}{ }_{4} \mathrm{Cr}\left(\mathrm{O}_{2}\right)_{\mathrm{ads}}$ & 5 & $\begin{array}{l}1-3=2.636 \\
1-4=2.599 \\
1-5=2.629 \\
2-3=2.901 \\
2-4=2.629 \\
2-5=2.449 \\
3-5=2.748 \\
3-4=2.630 \\
2-7=2.039 \\
5-6=1.919 \\
6-7=1.320\end{array}$ & $\begin{array}{l}\mathrm{Pt}_{1}=-0,192 \\
\mathrm{Pt}_{2}=-0,062 \\
\mathrm{Pt}_{3}=-0,109 \\
\mathrm{Pt}_{4}=-0,006 \\
\mathrm{Cr}_{5}=+0,888 \\
\mathrm{O}_{6}=-0,286 \\
\mathrm{O}_{7}=-0,233\end{array}$ & $\begin{array}{l}1-3-4=59.166 \\
1-4-3=60.528 \\
3-1-4=60.305 \\
2-3-4=56.523 \\
2-4-3=66.945 \\
3-2-4=56.531 \\
2-3-5=51.294 \\
2-5-3=67.567 \\
3-2-5=61.138 \\
1-3-5=58.421 \\
3-1-5=62.935\end{array}$ & $\begin{array}{c}1-5- \\
3=58.643 \\
1-4- \\
2=91.686 \\
4-2- \\
5=88.100 \\
2-5- \\
1=95.181 \\
4-1- \\
4=85.024 \\
2-7-6=104.818\end{array}$ & 983,26 \\
\hline $\left.\mathrm{J}_{2}\right)_{\text {ads }}$ & 6 & $\begin{array}{l}1-3=2.616 \\
1-4=2.616 \\
1-5=2.615 \\
2-3=2.679 \\
2-4=2.679 \\
2-5=2.522 \\
3-5=2.548 \\
4-5=2.548 \\
2-7=1.950 \\
5-6=1.860 \\
6-7=1.433\end{array}$ & $\begin{array}{l}\mathrm{Pt}_{1}=+0,036 \\
\mathrm{Pt}_{2}=-0,002 \\
\mathrm{Pt}_{3}=-0,189 \\
\mathrm{Pt}_{4}=-0,189 \\
\mathrm{~V}_{5}=+0,965 \\
\mathrm{O}_{6}=-0,284 \\
\mathrm{O}_{7}=-0,339\end{array}$ & $\begin{array}{l}1-3-2=89.032 \\
3-2-4=89.520 \\
2-4-1=89.033 \\
4-1-3=92.272 \\
1-3-5=60.843 \\
3-1-5=58.281 \\
1-5-3=60.874 \\
2-3-5=57.628 \\
3-2-5=58.572 \\
2-5-3=63.798 \\
2-4-5=57.628\end{array}$ & $\begin{array}{l}4-2-5=58.573 \\
2-5-4=63.798 \\
1-4-5=60.844 \\
4-1-5=58.282 \\
1-5-4=60.874 \\
2-5-6=77.176 \\
5-2-7=69.701\end{array}$ & 814,34 \\
\hline
\end{tabular}
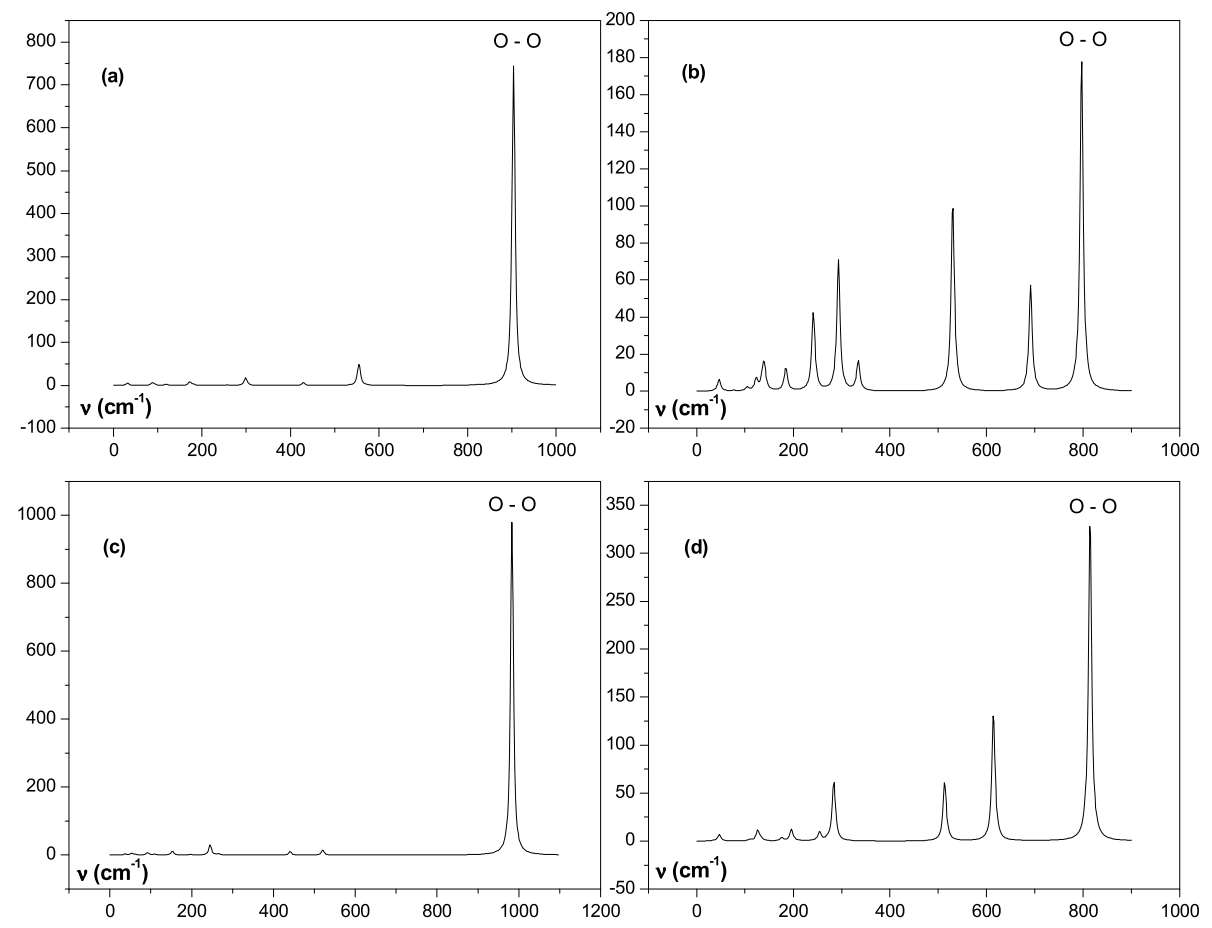

Figura 19: Espectros Teóricos de Infravermelho para as estruturas (a) $\mathrm{Pt}_{5}\left(\mathrm{O}_{2}\right)_{\text {ads }}$, (b) $\mathrm{Pt}_{4} \mathrm{Al}\left(\mathrm{O}_{2}\right)_{\text {ads }}$, (c) $\mathrm{Pt}_{4} \mathrm{Cr}\left(\mathrm{O}_{2}\right)_{\text {ads }}$ e (d) $\mathrm{Pt}_{4} \mathrm{~V}\left(\mathrm{O}_{2}\right)_{\text {ads. }}$ [B3LYP/LANL2DZ, 6-311G**]

A Tabela 16 apresenta as cargas atômicas e resultados das análises populacionais de Mulliken para as estruturas $\mathrm{Pt}_{5}\left(\mathrm{O}_{2}\right)_{\text {ads }}, \mathrm{Pt}_{4} \mathrm{Al}\left(\mathrm{O}_{2}\right)_{\text {ads }}, \mathrm{Pt}_{4} \mathrm{Cr}\left(\mathrm{O}_{2}\right)_{\text {ads }}$ e $\mathrm{Pt}_{4} \mathrm{~V}\left(\mathrm{O}_{2}\right)_{\text {ads. }}$ Como já foi abordado anteriormente, essa distribuição da população eletrônica fornece outras informações acerca do aumento no comprimento da 
ligação O—O resultando ou não na quebra da ligação e no estado final, peróxido ou superóxido, encontrado. Para efeito de comparação populações das estruturas antes da interação também foram colocados. As cargas atômicas de Mulliken mostram novamente um fluxo de carga no sentido dos clusters para os átomos de oxigênio. Nos resultados obtidos para $\mathrm{Pt}_{5}\left(\mathrm{O}_{2}\right)_{\text {ads }}$ as populações dos orbitais $\mathrm{p}$ dos átomos $\mathrm{Pt} 2$ e Pt4 são um pouco maiores após a interação com oxigênio. Os átomos Pt1 e Pt5 sofrem diminuições nas populações dos orbitais s enquanto que o átomo Pt3 sofre aumento na população dos orbitais $\mathrm{s}$. Além disso, observamos um decréscimo na população dos orbitais d dos átomos Pt2 e Pt4, enquanto que o as populações dos orbitais $p$ de ambos os átomos de oxigênio aumentam após a interação. As análises de NBO revelaram que para a ligação Pt1 - Pt4 o átomo Pt1 contribui de maneira mais expressiva com $78 \%$ [d(99\%)] e $22 \%$ de Pt4[s(13\%) + d(87\%)], forma-se aí uma ligação $\sigma_{d-d}$ e temos ainda para estes átomos uma ligação com contribuição invertida, ou seja, $22 \%$ de Pt1[d(99\%)] e $78 \%$ de Pt4 [s(13\%) + d(87\%)], formando o mesmo tipo de ligação; Entre Pt2 e O foi encontrado uma ligação onde observamos uma maior contribuição do oxigênio, sendo $38 \%$ de Pt2 $[(4 \%)+d(96 \%)]$ e $62 \%$ de $\mathrm{O} 7$ [s(3\%) + p(96\%)]; Entre Pt3 e Pt5 temos quase a mesma contribuição, sendo os orbitais d participando com 95\% para Pt3 e 84\% para Pt5 e ainda há outra ligação com contribuição exclusiva de Pt3(93\%) e Pt5 com 7\% e 75\% da densidade sobre seus orbitais 6s. Nota-se aqui que quando há contribuição dos orbitais s para o átomo metálico, a contribuição deste diminui consideravelmente na formação da ligação; A ligação O - O é uma ligação $\pi$ ocorrendo com contribuição de $50 \%$ de cada átomo, com densidade de $84 \%$ nos orbitais $p$ destes átomos. A transferência de carga dos orbitais d da platina para os orbitais $p$ do oxigênio não é suficiente para provocar a quebra da ligação $\mathrm{O}$ - $\mathrm{O}$. Entretanto, essa transferência provoca um 
alongamento e enfraquecimento da ligação $O$ - $O$, quando comparado com a ligação $\mathrm{O}=\mathrm{O}$ do oxigênio molecular.

Nos resultados para $\mathrm{Pt}_{4} \mathrm{Al}\left(\mathrm{O}_{2}\right)_{\text {ads }}$ observamos que as populações dos orbitais d do átomo Pt2 sofrem uma diminuição considerável enquanto que o orbital $p$ sobre aumento nas populações enquanto Pt1 sofre uma pequena diminuição na população dos orbitais s e ligeiro aumento na população dos orbitais d. Além disso, para o átomo Al5 foi observado que as populações dos orbitais $s$ e principalmente dos orbitais p sofrem diminuição das populações de Mulliken após interação com $\mathrm{O}_{2}$. Paralelamente a isso, os orbitais $p$ dos átomos de oxigênio sofrem aumento nas populações, principalmente o átomo 06 ligado à Al5, resultando assim no preenchimento dos orbitais antiligantes $\left(\pi_{\mathrm{g}}{ }^{*}\right)$ da molécula de oxigênio, causando a quebra da ligação $\mathrm{O}-\mathrm{O}$ e formando as ligações Pt2-O e Al5—O. Análises de NBO revelaram que no spin a temos 03 ligações: A primeira entre Pt1 e Al temos $46 \%$ de Pt $[s(94 \%)+p(6 \%)]$ e $54 \%$ de Al[s(69\%)+p(31\%)]; Em Pt3 - Pt4 temos uma ligação $\sigma_{s-s}$ com contribuição igual de cada átomo ( $97 \%$ orbitais s); e, por último, na ligação $\pi$ entre $\bigcirc$ - O temos $50 \%$ de contribuição de cada átomo numa ligação essencialmente formada pelos orbitais $p$ de cada átomo (90\%). Para o spin $\beta$ temos múltiplas formações oito de ligações: Em Pt1 - Pt3 e Pt1 - Pt4, as ligações são idênticas com contribuições de $62 \%$ de Pt1 [s(18\%)+ d (80\%)] e 38\% de Pt3(ou Pt4) [s(49\%) + d (49\%)]; Em Pt1 - Al há maior contribuição de Pt e formação de ligação $\sigma$ com $68 \%$ de $\mathrm{Pt} 1[\mathrm{~s}(58 \%)+\mathrm{d}(38 \%)]$ e $31 \%$ de $\mathrm{Al}[\mathrm{s}(40 \%)+\mathrm{p}(60 \%)]$; Entre Pt2-Pt3 e Pt2-Pt4 as ligações também são idênticas com 59\% de contribuição de Pt2 $[s(38 \%)+p(4 \%)+d(58 \%)]$ e $41 \%$ de Pt3 (ou Pt4) $[s(41 \%)+p(3 \%)+d(56 \%)] ; \mathrm{Na}$ Ligação Pt2 - O temos uma forte participação dos orbitais d da platina, sendo $35,6 \%$ de Pt2 $[s(17 \%)+d(82 \%)]$ e $64 \%$ de O [s(5\%)+p(94\%)]. A ligação Al - O é 
do tipo $\pi$ com pouca contribuição do átomo de $\mathrm{Al} 10 \%[\mathrm{~s}(48 \%)+\mathrm{p}(52 \%)]$ e $90 \%$ de $\mathrm{O}[\mathrm{s}(23 \%)+\mathrm{p}(77 \%)] ; \mathrm{Em} \mathrm{O}-\mathrm{O}$ as contribuições são de $50 \%$ para cada átomo formando uma ligação $p-p$ com $88 \%$ de participação destes orbitais.

Nos resultados para $\operatorname{Pt} t_{4} \mathrm{Cr}\left(\mathrm{O}_{2}\right)_{\text {ads }}$ observamos que as populações dos orbitais $s$ de todos os átomos Pt sofrem uma diminuições enquanto que o orbital $p$ dos átomos Pt2 e Pt3 sobrem um pequeno aumento nas populações. Além disso, os orbitais d dos átomos Pt1, Pt3 e Pt4 aumentam após a interação com oxigênio molecular. Para os átomos de oxigênio observamos acréscimos nas populações dos orbitais $p$, como resultado desta interação. Para compreender melhor as ligações existentes os resultados de NBO para os spins $\alpha$ e $\beta$ e revelaram que a ligação Pt2 Pt3 forma-se com maior contribuição de Pt2(91\%) com 98\% de seus orbitais d e Pt3 com seus orbitais $6 \mathrm{~s}(93 \%)$ formando uma ligação $\sigma_{s}$ - d; Para Pt2 - Cr temos $66 \%$ de Pt2 $[s(19 \%)+d(80 \%)]$ e $34 \%$ de $\operatorname{Cr}[s(18 \%)+d(78 \%)]$. Esta ligação é do tipo $d-d$; Em O - O a contribuição é igual para ambos os átomos, com $85 \%$ dos orbitais $p$ de cada átomo envolvidos formando uma ligação hibridizada, e temos ainda outra ligação O - O pura com 5\% a mais de contribuição de 05 sobre 06 sendo $99 \%$ de participação de orbitais p. No spin $\beta$ A ligação Pt1 - Pt3 ocorre com $54 \%$ de Pt1 $[s(45 \%)+d(53 \%)]$ e $46 \%$ de Pt3[s(48\%) + p(3\%) + d(49\%)]; Em Pt1 - Pt4 e Pt2 Pt4 o átomo Pt4 aumenta sua contribuição em aproximadamente $20 \%$ com participação de seus orbitais s indo de 27 a 41\%. Estas duas ligações são ambas do tipo $d$ - d, com diminuição dos orbitais naturais d para Pt4; Conectado ao átomo de $\mathrm{Cr}$, Pt tem $84 \%$ de participação na ligação $\mathrm{Pt}-\mathrm{Cr}$, sendo Pt2 [s(50\%)p + d(48\%)] e $16 \%$ de $\operatorname{Cr}[\mathbf{s}(20 \%)+p(10 \%)+d(69 \%)]$, esta é uma ligação sigma $s-d$; Ligado a Pt3, Pt4 contribui com $58 \%[s(29 \%)+d(70 \%)]$ formando uma ligação hibridizada; A 
última ligação para o spin beta é a ligação $\pi$ entre $O-O$, com participação igual para cada átomo sendo $05[s(14 \%)+p(86 \%)]$ e $06[s(16 \%)+p(84 \%)]$.

Tabela 16: Cargas atômicas e resultados das análises populacionais de Mulliken para as estruturas $\mathrm{Pt}_{5}, \mathrm{Pt}_{4} \mathrm{Al}, \mathrm{Pt}_{4} \mathrm{Cr}_{1}, \mathrm{Pt}_{4} \mathrm{~V}$ [B3LYP/LANL2DZ], $\mathrm{O}_{2}$ [B3LYP/6-311G**], $\mathrm{Pt}_{5}\left(\mathrm{O}_{2}\right)_{\text {ads }}, \mathrm{Pt}_{4} \mathrm{Al}\left(\mathrm{O}_{2}\right)_{\text {ads }}, \mathrm{Pt} \mathrm{Cr}_{4}\left(\mathrm{O}_{2}\right)_{\text {ads }}$ e $\mathrm{Pt}_{4} \mathrm{~V}\left(\mathrm{O}_{2}\right)_{\text {ads }}$ [B3LYP/LANL2DZ, 6-311G**].

\begin{tabular}{|c|c|c|c|c|c|c|c|c|}
\hline Estrutura & & $\mathrm{Pt}(1)$ & $\mathrm{Pt}(2)$ & $\mathrm{Pt}(3)$ & $\mathrm{Pt}(4)$ & $\mathrm{Pt}(5)$ & $\mathrm{O}(6)$ & $\mathrm{O}(7)$ \\
\hline \multirow[t]{4}{*}{$\mathrm{Pt}_{5}$} & Carga & $-0,04$ & 0,03 & 0,03 & 0,02 & $-0,04$ & - & - \\
\hline & $\mathrm{S}$ & 2.74 & 2,63 & 2.63 & 2.62 & 2.74 & _. & - \\
\hline & P & 6,15 & 6,27 & 6,27 & 6,21 & 6,15 & - & - \\
\hline & $\mathrm{D}$ & 9,15 & 9,07 & 9,07 & 9,15 & 9,15 & - & - \\
\hline \multirow[t]{4}{*}{$\mathrm{O}_{2}$} & Carga & - & - & & & & 0 & 0 \\
\hline & $\mathrm{s}$ & - & - & & & & 3,93 & 3,93 \\
\hline & $P$ & - & - & & & & 4,04 & 4,04 \\
\hline & D & - & - & & & & 0,03 & 0,03 \\
\hline \multirow[t]{4}{*}{$\mathrm{Pt}_{5}\left(\mathrm{O}_{2}\right)_{\mathrm{ads}}$} & Carga & 0,061 & 0,167 & 0,036 & 0,167 & 0,061 & $-0,246$ & $\begin{array}{c}- \\
0,246\end{array}$ \\
\hline & S & 2,65 & 2,62 & 2,72 & 2,62 & 2,65 & 3,95 & 3,95 \\
\hline & $P$ & 6,13 & 6,39 & 6,18 & 6,39 & 6,13 & 4,28 & 4,28 \\
\hline & D & 9,16 & 8,83 & 9,07 & 8,83 & 9,16 & 0,02 & 0,02 \\
\hline Estrutura & & $\mathrm{Pt}(1)$ & $\mathrm{Pt}(2)$ & $\mathrm{Pt}(3)$ & $\mathrm{Pt}(4)$ & $\mathrm{Al}(5)$ & $\mathrm{O}(6)$ & $\mathrm{O}(7)$ \\
\hline \multirow[t]{4}{*}{$\mathrm{Pt}_{4} \mathrm{Al}$} & jarga & $-0,256$ & $-0,256$ & $-0,256$ & $-0,256$ & 1,026 & - & - \\
\hline & $S$ & 2,93 & 2,93 & 2,93 & 2,93 & 1,08 & - & - \\
\hline & $\mathrm{P}$ & 6,24 & 6,24 & 6,24 & 6,24 & 6,24 & - & - \\
\hline & $D$ & 9,08 & 9,08 & 9,08 & 9,08 & 9,08 & - & - \\
\hline \multirow[t]{4}{*}{$\mathrm{O}_{2}$} & jarga & - & - & & & & 0 & 0 \\
\hline & $\mathrm{S}$ & - & - & & & & 3,93 & 3,93 \\
\hline & $P$ & - & - & & & & 4,04 & 4,04 \\
\hline & $D$ & - & - & & & & 0,03 & 0,03 \\
\hline \multirow[t]{4}{*}{$\mathrm{Pt}_{4} \mathrm{Al}\left(\mathrm{O}_{2}\right)_{\mathrm{ad}}$} & arga & $-0,241$ & $-0,092$ & $-0,153$ & $-0,153$ & 1,418 & $-0,476$ & $-0,302$ \\
\hline & $\mathrm{S}$ & 2,82 & 2,91 & 2,97 & 2,97 & 0,65 & 3,93 & 3,97 \\
\hline & $P$ & 6,26 & 6,41 & 6,15 & 6,15 & 0,93 & 4,52 & 4,31 \\
\hline & $\mathrm{D}$ & 9,15 & 8,77 & 9,04 & 9,04 & 0,00 & 0,01 & 0,01 \\
\hline Estrutura & & $\mathrm{Pt}(1)$ & $\mathrm{Pt}(2)$ & $\mathrm{Pt}(3)$ & $\mathrm{Pt}(4)$ & $\operatorname{Cr}(5)$ & $\mathrm{O}(6)$ & $\mathrm{O}(7)$ \\
\hline \multirow{4}{*}{$\mathrm{Pt}_{4} \mathrm{Cr}$} & Carga & $-0,186$ & $-0,186$ & $-0,196$ & $-0,196$ & 0,765 & - & - \\
\hline & $\mathrm{S}$ & 3,08 & 3,08 & 3,06 & 3,06 & 2,30 & - & - \\
\hline & $P$ & 6,19 & 6,19 & 6,19 & 6,19 & 6,22 & - & - \\
\hline & D & 8,92 & 8,92 & 8,94 & 8,94 & 4,71 & - & - \\
\hline \multirow[t]{4}{*}{$\mathrm{O}_{2}$} & Carga & - & - & & & & 0 & 0 \\
\hline & $\mathrm{S}$ & - & - & & & & 3,93 & 3,93 \\
\hline & $P$ & - & - & & & & 4,04 & 4,04 \\
\hline & D & - & - & & & & 0,03 & 0,03 \\
\hline \multirow[t]{4}{*}{$\mathrm{Pt}_{4} \mathrm{Cr}\left(\mathrm{O}_{2}\right)_{\mathrm{ad}}$} & Carga & $-0,192$ & $-0,062$ & $-0,109$ & $-0,006$ & 0,888 & $-0,287$ & $\begin{array}{c}- \\
0,233\end{array}$ \\
\hline & S & 2,92 & 2,88 & 2,75 & 2,77 & 2,24 & 3,91 & 3,95 \\
\hline & $P$ & 6,14 & 6,32 & 6,22 & 6,14 & 6,17 & 4,35 & 4,26 \\
\hline & D & 9,13 & 8,93 & 9,14 & 9,10 & 4,70 & 0,02 & 0,02 \\
\hline \multicolumn{2}{|l|}{ Estrutura } & $\operatorname{Pt}(1)$ & $\operatorname{Pt}(2)$ & $\mathrm{Pt}(3)$ & $\mathrm{Pt}(4)$ & $V(5)$ & $\mathrm{O}(6)$ & $\mathrm{O}(7)$ \\
\hline \multirow[t]{4}{*}{$\mathrm{Pt}_{4} \mathrm{~V}$} & Carga & $-0,184$ & $-0,184$ & $-0,184$ & $-0,184$ & 0,736 & - & - \\
\hline & $\mathrm{S}$ & 3,03 & 3,03 & 3,03 & 3,03 & 2,42 & - & - \\
\hline & $P$ & 6,19 & 6,19 & 6,19 & 6,19 & 6,28 & - & - \\
\hline & D & 8,97 & 8,97 & 8,97 & 8,97 & 3,61 & - & - \\
\hline \multirow[t]{4}{*}{$\mathrm{O}_{2}$} & Carga & - & - & & & & 0 & 0 \\
\hline & $\mathrm{S}$ & - & - & & & & 3,93 & 3,93 \\
\hline & $P$ & - & - & & & & 4,04 & 4,04 \\
\hline & D & - & - & & & & 0,03 & 0,03 \\
\hline $\mathrm{Pt}_{4} \mathrm{~V}\left(\mathrm{O}_{2}\right)_{\mathrm{ads}}$ & \& Carga & 0,037 & $-0,002$ & $-0,190$ & $-0,190$ & 0,965 & 0,340 & $-0,28$ \\
\hline
\end{tabular}




$\begin{array}{llllllll}\mathrm{S} & 2,75 & 2,87 & 3,07 & 3,08 & 2,29 & 3,93 & 3,96 \\ \mathrm{P} & 6,15 & 6,37 & 6,14 & 6,14 & 6,18 & 4,40 & 4,31 \\ \mathrm{D} & 9,06 & 8,75 & 8,97 & 8,97 & 3,56 & 0,01 & 0,02\end{array}$

Nos resultados para $\mathrm{Pt}_{4} \mathrm{~V}\left(\mathrm{O}_{2}\right)_{\text {ads }}$ observamos que as populações dos orbitais $\mathrm{s}$ dos átomos Pt1, Pt2 diminuem enquanto que há um aumento na população dos orbitais d de Pt1 e redução nos orbitais d de Pt2, pois parte dessa carga é transferida para o orbital $p$ do 07 . Além disso, para o átomo $V 5$ foi observado que as populações dos orbitais $s, p$ e d diminuem após interação com $\mathrm{O}_{2}$, ocorrendo transferência de carga para os orbitais $\mathrm{p}$ do $\mathrm{O6}$, resultando, assim, no preenchimento dos orbitais antiligantes $\left(\pi_{\mathrm{g}}{ }^{*}\right)$ da molécula de oxigênio, causando a quebra da ligação $\mathrm{O}-\mathrm{O}$ e formando as ligações Pt2-O e V5—O. Análises de NBO mostraram que no spin $\alpha$ Pt2 se liga a O com Pt contribuindo minoritariamente (25\%) com seus orbitais d em maioria (60\%). A ligação formada é $\sigma$ com $75 \%$ de contribuição do oxigênio com seus orbitais $p(90 \%)$; na ligação Pt2 - V a Platina contribui com maioria da densidade, sendo $78 \%$ de Pt2 [s(55\%) + d (45\%)] e $22 \%$ de $V[s(29 \%)+p(17 \%)+d(54 \%)] ;$ Em Pt3 $-V$ temos $87 \%$ de Pt3 $[s(3 \%)+d(97 \%)]$ e $13 \%$ de $\mathrm{V}[\mathrm{s}(16 \%)+p(31 \%)+\mathrm{d}(53 \%)]$ formando uma ligação $d$ com participação majoritária da Pt; em Pt4 $-\mathrm{V}$ temos $87 \%$ de Pt4 $[\mathrm{s}(3 \%)+\mathrm{d}(97 \%)]$ e $13 \%$ de $\mathrm{V}$ $[s(16 \%)+p(31 \%)+d(53 \%)]$; Nesta ligação ocorre o mesmo que na ligação anterior; Na ligação O—O a contribuição é igual para cada átomo com $88 \%$ dos orbitais $p$ formando uma ligação do tipo $\pi$. A ligação entre $\vee$ e um dos átomos de $O$ tem contribuição principalmente do átomo de $O(88 \%)$ com contribuição pura de seus orbitais $2 p$; Existe ainda uma ligação $\pi$ com $83 \%$ de $O[s(15 \%)+p(85 \%)]$ e $18 \%$ de $\mathrm{V}[\mathrm{s}(28 \%)+\mathrm{d}(72 \%)]$. No spin beta foram observadas 10 ligações: Pt1 - Pt3 tem natureza de $42 \%$ de Pt1[s(44\%) + d(56\%)] e $58 \%$ de Pt3[s(23\%) + d $(77 \%)]$. Esta 
ligação é do tipo d - d com contribuição eletrônica pouco maior por parte de Pt3; Para Pt1 - Pt4 as contribuições e a ligação é a mesma que em Pt1 - Pt3; Em Pt2 Pt3 as contribuições são iguais formando uma ligação com maior contribuição dos orbitais d do átomo Pt3 (58\% d Pt1: 78\% d Pt2). Entre os átomos Pt2 e Pt4 existem contribuições de 52 e 48\%, respectivamente, formando uma ligação d - d com participação maior destes orbitais para Pt4(78\%); Entre Pt2 e O há uma ligação d $\mathrm{p}$ hibridizada onde se percebe um aumento da participação dos orbitais d do átomo de Pt e contribuição de $94 \%$ dos orbitais $\mathrm{p}$ do átomo de Oxigênio; Em Pt3 - V o átomo de Pt tem participação de $80 \%$ na ligação com formação de hibridização $[(53 \%)+d(47 \%)]$ e $20 \%$ de $V[s(12 \%)+p(20 \%)+d(68 \%)]$; Entre Pt4 e V também a participação majoritária fica por conta do átomo de $\mathrm{Pt}$, também com $80 \%$ e contribuição de orbitais naturais dos dois átomos iguais à ligação anterior; $\mathrm{Na}$ ligação $\mathrm{O}-\mathrm{O}$, o átomo ligado ao $\mathrm{V}$ tem participação $2 \%$ maior com $88 \%$ de contribuição dos orbitais p para cada átomo, formando uma ligação $\pi_{p x-p x}$. Em $O-V$ o oxigênio tem $90 \%$ de participação na ligação com contribuição de seus orbitais p, e o átomo de $\mathrm{V}$ contribui com seus orbitais hibridizados com $84 \%$ em d; $\mathrm{A}$ última ligação no spin beta também acontece entre estes dois átomos com participação majoritária ainda do oxigênio sendo que agora há certa participação dos seus orbitais s $\mathrm{O}[\mathrm{s}(19 \%)+\mathrm{p}(81 \%)]$ e vanádio contribui com $14 \%[\mathrm{~s}(11 \%)+\mathrm{d}(87 \%)]$.

Para obtermos as barreiras de energias de dissociação da ligação $\mathrm{O}-\mathrm{O}$ após a interação com clusters $\mathrm{Pt}_{4} \mathrm{Al}$ e $\mathrm{Pt}_{4} \mathrm{~V}$, curvas de superfície de energia potencial foram calculadas e são apresentadas na Figura 20. Como pode ser observado, foi encontrado o valor de aproximadamente $0,62 \mathrm{eV}$ para a barreira de dissociação da ligação $\mathrm{O}-\mathrm{O}$ adsorvido sobre o cluster $\mathrm{Pt}_{4} \mathrm{Al}$ e aproximadamente $0,20 \mathrm{eV}$ para a barreira energética de dissociação da ligação $\mathrm{O}-\mathrm{O}$ adsorvido sobre o cluster $\mathrm{Pt}_{4} \mathrm{~V}$. 
Esses resultados mostram que a dissociação de $\mathrm{O}_{2}$ ocorre mais facilmente sobre o cluster $\mathrm{Pt}_{4} \mathrm{~V}$ que sobre o cluster $\mathrm{Pt}_{4} \mathrm{Al}$.

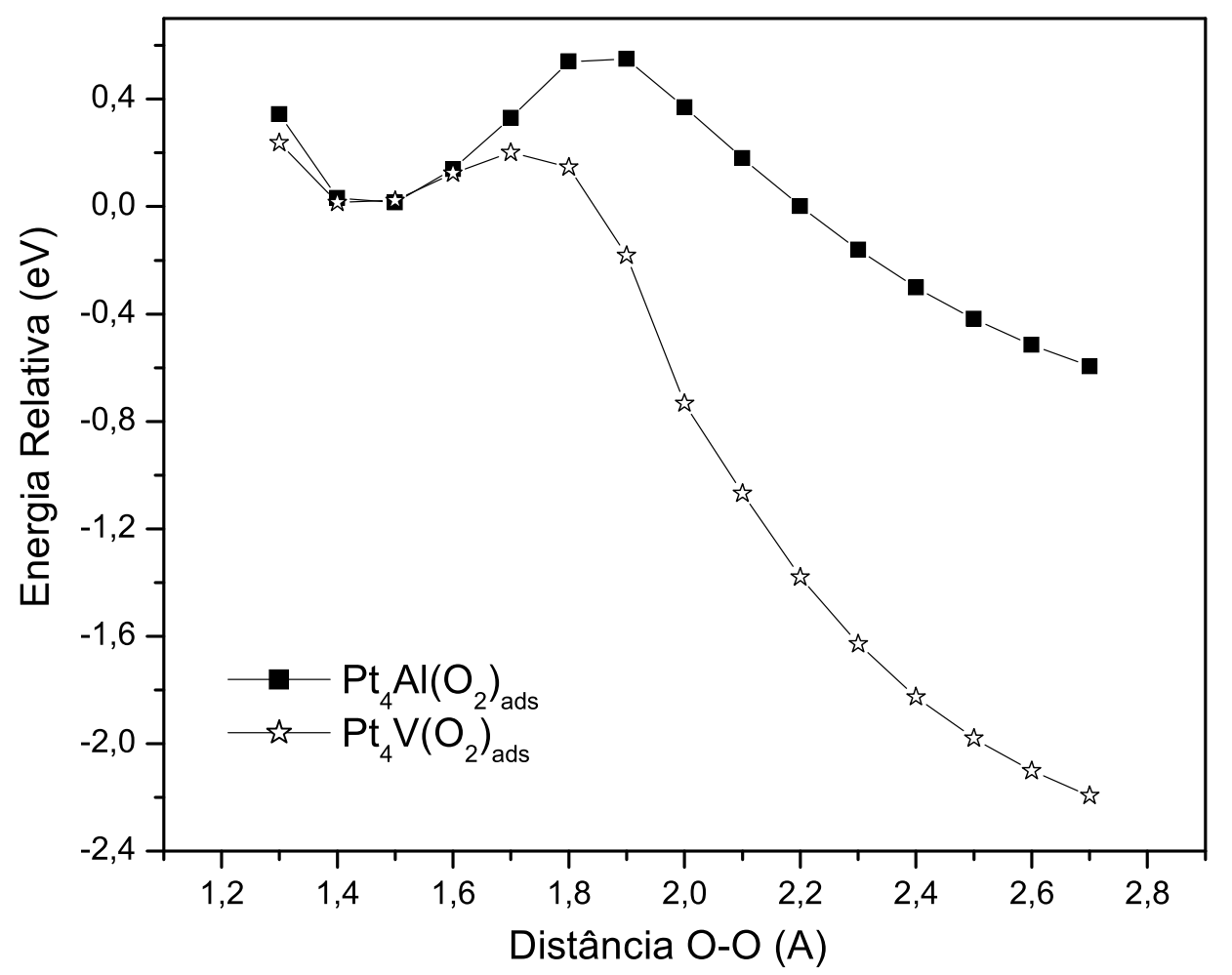

Figura 20: Curva de superfície de energia potencial com barreira energética para a dissociação de oxigênio molecular adsorvido em $\mathrm{Pt}_{4} \mathrm{Al}$ e $\mathrm{Pt}_{4} \mathrm{~V}$ [B3LYP/LANL2DZ, 6$311 \mathrm{G}^{\star *}$. Energias calculadas em relação às energias das estruturas otimizadas.

Os resultados encontrados para as energias de ligação entre oxigênio molecular e os clusters $\mathrm{Pt}_{5}, \mathrm{Pt}_{4} \mathrm{Al}, \mathrm{Pt}_{4} \mathrm{Cr}_{\text {e }} \mathrm{Pt}_{4} \mathrm{~V}$ são apresentados na Tabela 17, onde também estão inseridos os dados termodinâmicos, tais como variação de entalpia, variação de energia livre e variação de entropia a $298,15 \mathrm{~K}$ e 1 atm de pressão. Podemos observar, novamente, que a ligação torna-se mais forte quando o cluster $\mathrm{Pt}_{5}$ é dopado com alumínio, cromo ou vanádio. $\mathrm{O}$ valor da energia de ligação entre $\mathrm{Pt}_{5}$ e $\mathrm{O}_{2}$ foi de $-2,10 \mathrm{eV}$ e esse valor aumenta para $-3,05 \mathrm{eV},-2,95 \mathrm{eV}$ e $-4,03 \mathrm{eV}$ quando um átomo de platina é substituído por átomos de $\mathrm{Al}, \mathrm{Cr}$ e $\mathrm{V}$, respectivamente. Além disso, podemos observar que a adsorção de oxigênio sobre 
os clusters $\mathrm{Pt}_{4} \mathrm{Al}, \mathrm{Pt}_{4} \mathrm{Cr}$ e $\mathrm{Pt}_{4} \mathrm{~V}$ é uma reação muito exotérmica e envolve maior variação de entalpia e energia livre, que para a adsorção sobre o cluster $\mathrm{Pt}_{4} \mathrm{~V}$. Dentre os clusters estudados o $\mathrm{Pt}_{4} \mathrm{~V}$ foi o que apresentou maior calor de adsorção e maior variação de energia livre. Além disso, todas as reações apresentaram variações negativas de entropia e não foram observadas diferenças significativas na variação de entropia para adsorção de oxigênio.

Tabela 17: Energia de ligação, variação de entalpia, variação de energia livre e variação de entropia para a reação: $\mathrm{Pt}_{4} \mathrm{M}+\mathrm{O}_{2} \rightarrow \mathrm{Pt}_{4} \mathrm{M}\left(\mathrm{O}_{2}\right)_{\text {ads }}$. Dados Termodinâmicos obtidos a 298,15 K e p=1 atm. [B3LYP/LANL2DZ, 6-311G**].

$\begin{array}{ccccc}\text { Reação } & \mathbf{E}_{\text {Ligação }}(\mathbf{e V}) & \Delta \mathbf{H}\left(\mathbf{k c a l ~ m o l}^{-1}\right) & \Delta \mathbf{G}\left(\mathbf{k c a l ~ m o l}^{-1}\right) & \Delta \mathbf{S}\left(\mathbf{c a l ~ K}^{-1} \mathbf{m o l}^{-1}\right) \\ \mathrm{Pt}_{5}+\mathrm{O}_{2} \rightarrow \mathrm{Pt}_{5}\left(\mathrm{O}_{2}\right) \text { ads } & -2,10 & -48,75 & -36,90 & -39,74 \\ \mathrm{Pt}_{4} \mathrm{Al}+\mathrm{O}_{2} \rightarrow \mathrm{Pt}_{4} \mathrm{Al}\left(\mathrm{O}_{2}\right)_{\text {ads }} & -3,05 & -70,20 & -60,45 & -32,75 \\ \mathrm{Pt}_{4} \mathrm{Cr}+\mathrm{O}_{2} \rightarrow \mathrm{Pt}_{4} \mathrm{Cr}\left(\mathrm{O}_{2}\right) \text { ads } & -2,95 & -68,33 & -57,20 & -37,33 \\ \mathrm{Pt}_{4} \mathrm{~V}+\mathrm{O}_{2} \rightarrow \mathrm{Pt}_{4} \mathrm{~V}\left(\mathrm{O}_{2}\right) \text { ads } & -4,03 & -95,69 & -81,93 & -46,15\end{array}$




\section{CONCLUSÕES}

Os resultados obtidos para os clusters de platina dopados com $\mathrm{Al}, \mathrm{Cr}$ e $\mathrm{V}$ mostraram que os átomos de metais não nobres cedem carga para os átomos de platina e as análises de NBO revelaram que existe transferência de carga dos orbitais s dos átomos $\mathrm{Cr}$ e $\mathrm{V}$ para orbitais d da platina e transferência de carga dos orbitais s e p do Al para os orbitais d da platina. Em todos os clusters estudados, quando um átomo de platina é substituído por $\mathrm{Cr}$ ou $\mathrm{V}$ os valores dos potenciais verticais de ionização decrescem quando comparados com os clusters Pt. Por outro lado, com exceção do cluster Pt-Al, todos os clusters dopados com alumínio mostraram aumento no potencial vertical de ionização. A diminuição no potencial vertical de ionização favorece a transferência do cluster para o adsorbato, provocando uma alteração na reatividade para a adsorção e dissociação de $\mathrm{O}_{2}$ sobre estes clusters.

Os estudos teóricos para interação entre oxigênio molecular e os clusters $\mathrm{Pt}_{2}$, Pt-Al, Pt-Cr e Pt-V mostram que ocorre adsorção dissociativa de oxigênio molecular sobre os clusters $\mathrm{Pt}_{2}, \mathrm{Pt}-\mathrm{Cr}$ e $\mathrm{Pt}-\mathrm{V}$, onde observamos uma adsorção que segue o modelo de ponte (Modelo Yeager) enquanto que sobre o cluster Pt-Al a adsorção segue o modelo de Pauling, com o oxigênio molecular adsorvido em apenas um sítio do cluster, sem a quebra da ligação O-O. As análises das populações de Mulliken e de NBO para a interação entre $\mathrm{O}_{2}$ e $\mathrm{Pt}_{2}$, $\mathrm{Pt}-\mathrm{Cr}$ e $\mathrm{Pt}-\mathrm{V}$ revelaram que ocorre transferência de carga dos orbitais $s$ e $d$ dos metais para os orbitais $p$ do oxigênio, resultando no preenchimento dos orbitais antiligantes da molécula de oxigênio, provocando a quebra da ligação O—O e formação de ligações hibridizadas Metal O. Além disso, observamos que a adsorção de oxigênio molecular torna-se mais forte quando um átomo de platina é substituído por outro metal. A adsorção de 
oxigênio sobre os clusters Pt-Al, Pt-Cr e Pt-V é uma reação exotérmica e envolve maior variação de entalpia e energia livre, quando comparado com a adsorção no cluster $\mathrm{Pt}_{2}$. Dentre os clusters diatômicos estudados o Pt-V foi o que apresentou maior energia de adsorção e maior variação de energia livre. Curvas de superfície de energia potencial revelaram um valor de aproximadamente 1,0 eV para a barreira de dissociação da ligação $\mathrm{O}$ - O para oxigênio molecular adsorvido em $\mathrm{Pt}_{2}$. Sobre $\mathrm{Pt}-\mathrm{Cr}$ e Pt-V, esse valor decresce para $0,56 \mathrm{eV}$ e $0,20 \mathrm{eV}$, respectivamente. Esses resultados mostram que a dissociação de $\mathrm{O}_{2}$ ocorre mais facilmente sobre esses clusters que sobre o cluster $\mathrm{Pt}_{2}$.

Os estudos da interação entre oxigênio molecular e os clusters $\mathrm{Pt}_{3}, \mathrm{Pt}_{2} \mathrm{Al}$, $\mathrm{Pt}_{2} \mathrm{Cr}$ e $\mathrm{Pt}_{2} \mathrm{~V}$ mostram que ocorre adsorção não dissociativa de oxigênio molecular sobre o cluster $\mathrm{Pt}_{3}$ onde observamos uma adsorção segundo o modelo de ponte enquanto que sobre os clusters $\mathrm{Pt}_{2} \mathrm{Al}$ e $\mathrm{Pt}_{2} \mathrm{~V}$ ocorre adsorção dissociativa de oxigênio molecular sobre os clusters. Por outro lado, sobre o cluster $\mathrm{Pt}_{2} \mathrm{Cr}$ a adsorção segue o modelo de Pauling, com o oxigênio molecular adsorvido em apenas um sítio do cluster, que foi o átomo Pt, sem a quebra da ligação O—O. As análises das populações de Mulliken e de $\mathrm{NBO}$ para a interação entre $\mathrm{O}_{2} \mathrm{e} \mathrm{Pt}_{3}$, mostraram que existe transferência de carga dos orbitais d dos átomos de platina ligados aos átomos de oxigênio para os orbitais $p$ do oxigênio, resultando em ligações Pt-O hibridizadas, com maior contribuição dos orbitais d, e enriquecimento dos orbitais $p$ do oxigênio, mas sem a quebra de ligação $O$ - O. Para a interação entre $\mathrm{Pt}_{2} \mathrm{Al}$ e oxigênio molecular, observamos que as populações dos orbitais $\mathrm{s}$ e $\mathrm{d}$ do átomo Pt1, ligado ao oxigênio, sofrem uma diminuição considerável enquanto que o orbital $\mathrm{p}$ sobre aumento nas populações. Por outro lado, para o átomo $\mathrm{Pt}_{2}$ foi observado uma diminuição na população dos orbitais $s$ e $p$ e um aumento 
considerável na população dos orbitais d. Além disso, para o átomo Al3 foi observado que as populações dos orbitais $s$ diminuem praticamente a metade enquanto que os orbitais $p$ sofrem aumento na população de Mulliken após interação com $\mathrm{O}_{2}$. Paralelamente a isso, os orbitais $\mathrm{p}$ dos átomos de oxigênio sofrem aumento nas populações, principalmente o átomo $\mathrm{O} 5$ ligado à Al3. Essa transferência eletrônica resultou no preenchimento dos orbitais antiligantes $\left(\pi_{\mathrm{g}}{ }^{*}\right)$ da molécula de oxigênio, sendo suficiente para causar a quebra da ligação $\mathrm{O}-\mathrm{O}$ e formação das ligações hibridizadas entre $\mathrm{Pt} 1-\mathrm{O} 4$ e $\mathrm{Al} 3-\mathrm{O} 5$. Enquanto isso, na interação entre $\mathrm{Pt}_{2} \mathrm{~V}$ e oxigênio molecular, observou-se que as populações dos orbitais $\mathrm{s}$ do átomo Pt1 e dos orbitais $s$ e d de Pt2 diminuem enquanto que há um aumento na população dos orbitais d de Pt1 e nos orbitais $p$ de Pt2. Com isso, podemos concluir que o fluxo de carga acontece dos orbitais $s$ e d de Pt2 o orbital p do O5. Além disso, para o átomo $\mathrm{V} 3$ foi observado que as populações dos orbitais s e d diminuem após interação com $\mathrm{O}_{2}$, ocorrendo transferência de carga para os orbitais p do $\mathrm{O}$. Essa transferência de carga dos orbitais s e d de Pt2 e V3 resulta no preenchimento dos orbitais antiligantes $\left(\pi_{\mathrm{g}}{ }^{*}\right)$ da molécula de oxigênio, causando a quebra da ligação $\mathrm{O}-\mathrm{O}$ e formação das ligações $\mathrm{Pt2}-\mathrm{O} 5$ e V3-O4. Nos resultados para $\mathrm{Pt} 2 \mathrm{Cr}\left(\mathrm{O}_{2}\right)_{\text {ads }}$ observamos que as populações dos orbitais $d$ dos dois átomos Pt sofrem uma diminuições enquanto que o orbital $p$ do átomo Pt1 e s do átomo Pt2 apresentaram aumentos nas populações após interação com oxigênio molecular. Além disso, a população dos orbitais s do átomo $\mathrm{Cr} 3$ diminuiu de 2,45 para 2,31 enquanto que dos orbitais d apresentaram considerável aumento de 4,01 para 4,67 após a interação com oxigênio. Por outro lado, para os átomos de oxigênio observamos acréscimos nas populações dos orbitais $p$, como resultado desta interação. Curvas de superfície de energia potencial para a dissociação de oxigênio molecular sobre $\mathrm{Pt}_{2} \mathrm{Al}$ e $\mathrm{Pt}_{2} \mathrm{~V}$ 
mostraram um valor de aproximadamente $0,21 \mathrm{eV}$ para a barreira de dissociação da ligação $\mathrm{O}-\mathrm{O}$ adsorvido sobre o cluster $\mathrm{Pt}_{2} \mathrm{Al}$ e aproximadamente $0,30 \mathrm{eV}$ para a barreira energética de dissociação da ligação $\mathrm{O}-\mathrm{O}$ adsorvido sobre o cluster $\mathrm{Pt}_{2} \mathrm{~V}$. Com esses resultados concluímos que a dissociação de $\mathrm{O}_{2}$ ocorre mais facilmente sobre o cluster $\mathrm{Pt}_{2} \mathrm{Al}$ que sobre o cluster $\mathrm{Pt}_{2} \mathrm{~V}$.

Os estudos da interação entre oxigênio molecular e os clusters $\mathrm{Pt}_{4}, \mathrm{Pt}_{3} \mathrm{Al}$, $\mathrm{Pt}_{3} \mathrm{Cr}$ e $\mathrm{Pt}_{3} \mathrm{~V}$ mostram que ocorre adsorção não dissociativa de oxigênio molecular sobre os clusters $\mathrm{Pt}_{4}$ e $\mathrm{Pt}_{3} \mathrm{Cr}$ onde foi observado que a adsorção segue o modelo de ponte, com freqüências de estiramento de $933,66 \mathrm{~cm}^{-1}$ e $1087,11 \mathrm{~cm}^{-1}$ para a ligação $\mathrm{O}$ - $\mathrm{O}$ do oxigênio adsorvido sobre $\mathrm{Pt}_{4}$ e $\mathrm{Pt}_{3} \mathrm{Cr}$, respectivamente. Por outro lado, sobre os clusters $\mathrm{Pt}_{3} \mathrm{Al}_{\mathrm{I}}$ e $\mathrm{Pt}_{3} \mathrm{~V}$ a adsorção de oxigênio molecular também seguiu o modelo ponte, com dissociação da ligação $O$ - $O$. As análises das populações de Mulliken e de NBO para a interação entre $\mathrm{O}_{2}$ e $\mathrm{Pt}_{4}$, mostraram que as populações dos orbitais $\mathrm{d}$ do átomo Pt2 e dos orbitais $\mathrm{s}$ e d do átomo Pt3 diminuem após a adsorção. Além disso, houve um aumento nas populações dos orbitais $s$ do átomo Pt1 e diminuições nas populações dos orbitais d do átomo Pt1 e dos orbitais $p$ e d do átomo Pt4. Por outro lado, as populações dos orbitais $p$ dos átomos de oxigênio aumentam a interação com $\mathrm{O}_{2}$, o que não foi suficiente para a quebra da ligação $\mathrm{O}$ O. Para a interação entre $\mathrm{Pt}_{3} \mathrm{Al}$ e oxigênio molecular, as populações dos orbitais $d$ do átomo Pt3 sofrem uma diminuição considerável enquanto que o orbital $s$ sofre aumento nas populações. Enquanto isso, o átomo Pt1 sofre uma pequena diminuição na população dos orbitais $p$ e pequeno aumento na população dos orbitais s e d. Além disso, para o átomo Al4 foi observado que as populações dos orbitais s diminuem em cerca de $60 \%$ enquanto que as populações do orbital $p$ sofrem acréscimos nas populações de Mulliken após interação com $\mathrm{O}_{2}$. 
Paralelamente a isso, os orbitais $p$ dos dois átomos de oxigênio sofrem aumento nas populações, principalmente $\mathrm{O}$ átomo $\mathrm{O} 5$ ligado à $\mathrm{Al} 4$, resultando assim no preenchimento dos orbitais antiligantes $\left(\pi_{\mathrm{g}}{ }^{*}\right)$ da molécula de oxigênio, causando a quebra da ligação O—O e formando as ligações Pt3-O6 e Al4—O5. Nos resultados para $\mathrm{Pt}_{3} \mathrm{Cr}\left(\mathrm{O}_{2}\right)_{\text {ads }}$ observamos que as populações dos orbitais $d$ de todos os átomos Pt sofrem uma diminuições enquanto que os orbitais $p$ do átomo Pt2 sobrem um pequeno aumento nas populações. Por outro lado, as populações dos orbitais $p$ e d do átomo $\mathrm{Cr}$ diminuem após a interação com oxigênio molecular. Além disso, observamos acréscimos nas populações dos orbitais $p$ dos átomos de oxigênio como resultado desta interação. Nos resultados para $\mathrm{Pt}_{3} \mathrm{~V}\left(\mathrm{O}_{2}\right)_{\mathrm{ads}}$ observamos que as populações dos orbitais $s$ dos átomos $\mathrm{Pt} 2$, Pt3 diminuem enquanto que há um aumento na população dos orbitais d de Pt3 e diminuição nos orbitais d de Pt2, pois parte dessa carga é transferida para o orbital $p$ de O5. Além disso, para o átomo V4 foi observado que as populações dos orbitais s e d diminuem após interação com $\mathrm{O}_{2}$, ocorrendo transferência de carga para os orbitais $p$ do $\mathrm{O}$, resultando, assim, no preenchimento dos orbitais antiligantes $\left(\pi_{\mathrm{g}}{ }^{*}\right)$ da molécula de oxigênio, causando a quebra da ligação O—O e formando as ligações Pt2-O5 e V4-O6. Curvas de superfície de energia potencial para a dissociação de oxigênio molecular sobre $\mathrm{Pt}_{3} \mathrm{Al}$ e $\mathrm{Pt}_{3} \mathrm{~V}$ mostraram um valor de aproximadamente $0,46 \mathrm{eV}$ para a barreira de dissociação da ligação $\mathrm{O}-\mathrm{O}$ adsorvido sobre o cluster $\mathrm{Pt}_{3} \mathrm{Al}$ e aproximadamente $0,28 \mathrm{eV}$ para a barreira energética de dissociação da ligação $\mathrm{O}-\mathrm{O}$ adsorvido sobre o cluster $\mathrm{Pt}_{3} \mathrm{~V}$. Com esses resultados podemos concluir que a dissociação da ligação $\mathrm{O}$ - O ocorre mais facilmente sobre o cluster $\mathrm{Pt}_{3} \mathrm{~V}$ que sobre o cluster $\mathrm{Pt}_{3} \mathrm{Al}$.

Os estudos para interação entre oxigênio molecular e os clusters $\mathrm{Pt}_{5}, \mathrm{Pt}_{4} \mathrm{Al}$, $\mathrm{Pt}_{4} \mathrm{Cr}$ e $\mathrm{Pt}_{4} \mathrm{~V}$ revelaram que ocorre adsorção dissociativa de oxigênio molecular 
sobre os clusters $\mathrm{Pt}_{4} \mathrm{Al}$ e $\mathrm{Pt}_{4} \mathrm{~V}$, onde observamos uma adsorção que segue o modelo de ponte (Modelo Yeager) enquanto que sobre 0 cluster $\mathrm{Pt}_{5}$ a adsorção segue 0 modelo de ponte sem dissociação da ligação O—O. Por outro lado, a adsorção sobre $\mathrm{Pt}_{4} \mathrm{Cr}$ segue o modelo de Pauling, com o oxigênio molecular adsorvido em apenas um sítio do cluster, sem a quebra da ligação $\mathrm{O}-\mathrm{O}$. As análises de NBO revelaram na estrutura $\mathrm{Pt} 5\left(\mathrm{O}_{2}\right)_{\text {ads }}$ um decréscimo na população dos orbitais d dos átomos $\mathrm{Pt} 2$ e $\mathrm{Pt} 4$, enquanto que o as populações dos orbitais $\mathrm{p}$ de ambos os átomos de oxigênio aumentam após a interação. Nos resultados para $\mathrm{Pt}_{4} \mathrm{Al}\left(\mathrm{O}_{2}\right)_{\text {ads }}$ observamos que as populações dos orbitais d do átomo Pt2 sofrem uma diminuição considerável enquanto que o orbital p sobre aumento nas populações enquanto Pt1 sofre uma pequena diminuição na população dos orbitais s e ligeiro aumento na população dos orbitais d. Além disso, para o átomo Al5 foi observado que as populações dos orbitais $s$ e principalmente dos orbitais $p$ sofrem diminuição das populações de Mulliken após interação com $\mathrm{O}_{2}$. Paralelamente a isso, os orbitais $p$ dos átomos de oxigênio sofrem aumento nas populações, principalmente o átomo O6 ligado à Al5, resultando assim no preenchimento dos orbitais antiligantes $\left(\pi_{g}{ }^{*}\right)$ da molécula de oxigênio, causando a quebra da ligação $\mathrm{O}-\mathrm{O}$ e formando as ligações hibridizadas $\mathrm{Pt} 2-\mathrm{O}$ e $\mathrm{Al} 5-\mathrm{O}$. Nos resultados para $\mathrm{Pt} 4 \mathrm{Cr}\left(\mathrm{O}_{2}\right)_{\text {ads }}$ observamos que as populações dos orbitais $s$ de todos os átomos Pt sofrem uma diminuições enquanto que o orbital $p$ dos átomos $\mathrm{Pt} 2$ e Pt3 sobrem um pequeno aumento nas populações. Além disso, os orbitais d dos átomos Pt1, Pt3 e Pt4 aumentam após a interação com oxigênio molecular. Para os átomos de oxigênio observamos acréscimos nas populações dos orbitais $p$, como resultado desta interação. Nos resultados para $\mathrm{Pt}_{4} \mathrm{~V}\left(\mathrm{O}_{2}\right)_{\text {ads }}$ observamos que as populações dos orbitais $s$ dos átomos $\mathrm{Pt1}$, Pt2 diminuem enquanto que há um aumento na população dos orbitais d de Pt1 e 
redução nos orbitais d de $\mathrm{Pt2}$, pois parte dessa carga é transferida para o orbital $\mathrm{p}$ do 07 . Além disso, para o átomo V5 foi observado que as populações dos orbitais s, p e d diminuem após interação com $\mathrm{O}_{2}$, ocorrendo transferência de carga para os orbitais $p$ do 06 , resultando, assim, no preenchimento dos orbitais antiligantes $\left(\pi_{\mathrm{g}}{ }^{*}\right)$ da molécula de oxigênio, causando a quebra da ligação $\mathrm{O}-\mathrm{O}$ e formando as ligações hibridizadas entre Pt2-O e V5-O, envolvendo maiores contribuições dos orbitais d dos átomos Pt e V. Curvas de superfície de energia potencial mostraram um valor de aproximadamente $0,62 \mathrm{eV}$ para a barreira de dissociação da ligação $\mathrm{O}-\mathrm{O}$ adsorvido sobre o cluster $\mathrm{Pt}_{4} \mathrm{Al}$ e aproximadamente $0,20 \mathrm{eV}$ para a barreira energética de dissociação da ligação $\mathrm{O}-\mathrm{O}$ adsorvido sobre o cluster $\mathrm{Pt}_{4} \mathrm{~V}$. com esses resultados podemos concluir que a dissociação de $\mathrm{O}_{2}$ ocorre mais facilmente sobre o cluster $\mathrm{Pt}_{4} \mathrm{~V}$ que sobre o cluster $\mathrm{Pt}_{4} \mathrm{Al}$.

Estes resultados são necessários para entender os fatores importantes que governam a atividade catalítica frente à reação de redução de oxigênio sobre os sítios de adsorção dos clusters de platina copados com cromo, vanádio e alumínio, uma vez que a forma e natureza da adsorção de oxigênio sobre a superfície do catalisador é um fator que pode favorecer a redução diretamente à água (mecanismo $4 \mathrm{e}^{-}$) ou favorecer o mecanismo $2 \mathrm{e}^{-}$, resultando como produto o peróxido de hidrogênio. 


\section{REFERÊNCIAS}

1. CHAVES, J. A. P; ARAÚJO, M. F. A.; VARELA JÚNIOR, J. de J. G.; TANAKA, A. A. Eletrocatálise da reação de redução de oxigênio sobre eletrodos de grafite modificados com ftalocianina tetracarboxilada de ferro, Eclética Química, v.28, p.920, 2003.

2. SANTOS JR, J. R. Propriedades eletroquímicas de ftalocianinas de ferro e cobalto para a redução de oxigênio, Dissertação de Mestrado, IQSC/USP, São Carlos-SP, 1991.

3. MACHADO, S. A. S.; TANAKA, A. A.; GONZALEZ, E. R., Underpotential deposition of lead on polycrystalline platinum and its influence on the oxygen reduction reaction, Electrochimica Acta, v.39, p.2591-2597. 1994.

4. PAFFET M. T., BEERY, J. G.; GOTTESFELD, S. Oxygen reduction at $\mathrm{Pt}_{0.65} \mathrm{Cr}_{0.35}, \mathrm{Pt}_{0.2} \mathrm{Cr}_{0.8}$ and roughened platinum, Journal of the Electrochemical Society v.135, p.1431-1436, 1988.

5. LIMA, F. H. B., GIZ, M. J., TICIANELLI, E. A. Electrochemical performance of disperse $\mathrm{Pt}-\mathrm{M}(\mathrm{M}=\mathrm{V}, \mathrm{Cr}$ and $\mathrm{Co})$ nanoparticles for the oxygen reduction electrocatalysis, Jounal of the Brazilian Chemical Society, v. 26 (3A), p.328-336, 2005.

6. TODA, T.; IGARASHI, H.; UCHIDA, H.; WATANABE, M.; Enhancement of the electroreduction of oxygen on $\mathrm{Pt}$ alloys with $\mathrm{Fe}, \mathrm{Ni}$, and $\mathrm{Co}$ Journal of The Electrochemical Society, v.146, p.3750-3756, 1999.

7. LIMA, F. H. B., TICIANELLI, E. A. Oxygen Electrocatalysis on ultra-thin porous coating rotating ring/disk electrode in alkaline media. Electrochimica Acta, v. 49., p. 4091-4099, 2004. 
8. YEAGER, E. B. Electrocatalysts for $\mathrm{O}_{2}$ reduction, Electrochimica Acta, v. 29, p.1527-1537, 1984.

9. YEAGER, E. B. Dioxygen electrocatalysis - mechanisms in relation to catalyst structure, Journal of Molecular Catalalysis, v. 38, p. 5-25, 1986.

10. ADZIC, R. in: J. Lipkouski, P. N. Ross (Eds.), Electrocatalysis, Wiley-VCH, New York, p.209,1998.

11. TICIANELLI, E. A.; GONZALEZ, E. R.; Eletroquímica: Princípios e Aplicações, EDUSP, São Paulo, 1998.

12. DAMJANOVIC, A.; BRUSIC, V.; BOCKRIS, J. O. M. Mechanism of Oxygen Reduction Related to Electronic Structure of Gold-Palladium Alloy, Journal of Physical Chemistry, v.71, p.2471-2472, 1967.

13. JALAN, V.; TAYLOR, J.; Importance of interatomic spacing in catalytic reduction of oxygen in phosphoric-acid, Jounal of the Electrochemical Society, $v$. 130, p.2299-2301, 1983.

14. APLEBBY, A. J.; Molten carbonate fuel cells in a future United Kingdom context, Energy, v.13, p.97-106, 1988.

15. MUKERJEE, S.; SRINIVASAN, S.; Enhanced electrocatalysis of oxygen reduction on platinum alloys in proton- exchange membrane fuel-cells, Journal of Electroanalytical Chemistry, , v.357, p.201-224, 1993.

16. GLASS, J. T.; CAHEN, G. L.; STONER, G. E. The effect of metallurgical variables on the electrocatalytic properties of $\mathrm{PtCr}$ alloys, Journal of the Electrochemical Society, v.134, p.58-65, 1987. 
17. PAFFET, M. T.; BERRY G.J., GOTTESFELD, S.; Oxygen reduction at $\mathrm{Pt}_{0,65} \mathrm{Cr}_{0,35}, \mathrm{Pt}_{0,2} \mathrm{Cr}_{0,8}$ and roughened platinum, Journal of the Electrochemical Society, v.135, p.1431-1436, 1988.

18. BEARD, B.C., ROSS, P.N., The structure and activity of Pt-Co alloys as oxygen reduction electrocatalysts, Journal of The Electrochemical Society, v.137, p.3368-3374, 1990.

19. TODA, T; IGARASHI, H.; UCHIDA, H.; WATANABE, M., Enhancement of the electroreduction of oxygen on $\mathrm{Pt}$ alloys with $\mathrm{Fe}, \mathrm{Ni}$, and $\mathrm{Co}$, Journal of the Electrochemical Society, v.146, p. 3750-3756, 1996.

20. MUKERJEE, S; SRINIVASAN, S.; SORIAGA, M.P.; MCBREEN, J, Role of structural and electronic-properties of $\mathrm{pt}$ and $\mathrm{Pt}$ alloys on electrocatalysis of oxygen reduction - an in-situ XANES and EXAFS investigation, Journal of the Electrochemical Society, v.142, p. 1409-1422, 1995.

21. ARICÓ, A. S.; SHUKLA, A. K.; KIM, H.; PARK, S.; MIN, M. ANTONUCCI, V.; An XPS study on oxidation states of $\mathrm{Pt}$ and its alloys with $\mathrm{Co}$ and $\mathrm{Cr}$ and its relevance to electroreduction of oxygen, Applied Surface Science, v.172, p.33-40, 2001.

22. STAMENKOVIC, V.; SCHMIDT, T. J.; ROSS, P. N.; MARKOVIC N.M.; Surface composition effects in electrocatalysis: Kinetics of oxygen reduction on well-defined $\mathrm{Pt}_{3} \mathrm{Ni}$ and $\mathrm{Pt}_{3} \mathrm{Co}$ alloy surfaces, Journal of Physical Chemistry B, v. 106, p.1197011979, 2002.

23. LEVINE, I. N. Quantum Chemistry. 4.ed. New York: Prentice Hall, 1991. 628p.

24. KOCH, W.; HOLTHAUSEN, M. C., A chemist's Guide to density functional theory, 2nd ed., Willey-VCH: Weinheim, 2001. 
25. DRUDE, P. Zur Elektronentheorie der Metalle; II. Teil. Galvanomagnetische und thermomagnetische Effect. Annalen der Physik, v. 308, p. 369-402, 1900.

26. THOMAS, P. H. The Calculations of Atomic Fields, Mathematical Proceedings of the Cambridge Philosophical Society, v.23, p.542-548, 1927.

27. FERMI, E. Um Metodo Statistico per La Determinazione di Alcune Proprietà Dell'Atomo, Rend. Lincei, v. 6, p. 602-607, 1927.

28. VIANNA, J. D. M., FAZZIO, A., CANUTO, S., Teoria quântica de moléculas e sólidos: simulação computacional, Ed. Livraria da Física: São Paulo, 2004.

29. HOHENBERG, P., KOHN, W., Inhomogeneous Electron Gas, Physical Review, v.136, p.B864-B871, 1964.

30.KOHN, W., SHAM, L., Self-Consistent Equations Including Exchange and Correlation Effects, Physical Review, v.140, A1133-A1138, 1965.

31.PARR, R.G. e YANG, W. Density Functional Theory of Atoms and Molecules. Oxford: Oxford University Press, 1989. 352 p.

32. VOSKO, S. H.; WILK, L.; NUSAIR, M., Accurate spin-dependent electron liquid correlation energies for local spin density calculations: a critical analysis, Canadian Journal of Physics, v. 58, p. 1200-1211, 1980.

33.PERDEW, J. P.; ZUNGER, A., Self-interaction correction to density-functional approximations for many-electron systems. Physical Review B, v.23, p.50485079, 1981.

34.PARR, R.G. e YANG, W. Density Functional Theory of Atoms and Molecules. Oxford: Oxford University Press, 1989. 352 p. 
35. CAPELLE, K., A bird's eyes view of density-functional theory., Brazilian Journal of Physics, v. 36, p. 1318-1343, 2006.

36.PERDEW, J. P., BURKE, K. ERNZERHOF, M., Generalized gradient approximation made simple., Physical Review Letters, v. 77, p. 3865-3868, 1996.

37.BECKE, A. D., Density-functional Exchange-energy aproximation with correct asymptotic behavior., Physical Review A, v. 38, p. 3098-3100, 1988.

38. LEE, C.; YANG, W.; PARR, R.G.; Development of the Colle-Salvetti correlation-energy formula into a functional of the electron density, Physical Review B, v.37, p.785-789, 1988.

39. BECKE, A. D., Density-functional thermochemistry. V. Systematic optimization of exchange-correlation functionals, Journal of Chemical Physics, v.107, p.85548560, 1997.

40. HAMMER, B.; NØRSKOV, J. K.; Theoretical surface science and catalysiscalculations and concepts, Advances in Catalysis, v.45, p.71-129, 2000.

41. EICHLER, A.; HAFNER, J.; Molecular Precursors in the Dissociative Adsorption of $\mathrm{O}_{2}$ on Pt(111) Physical Review Letters, v.79, p.4481-4484, 1997.

42. HYMAN, M. P. MEDLIN, P. W. Theoretical study of the adsorption and dissociation of oxygen on $\mathrm{Pt}(111)$ in the presence of homogeneous electric fields. Journal of Physical Chemistry B, v.109, p.6304-6310, 2005.

43. FEIBELMAN, P. J., ESCH, S. and MICHELY, T.; O Binding Sites on Stepped Pt(111) Surfaces, Physical Review Letters, v.77, p.2257-2260, 1996. 
44.XU, Y.; RUBAN, A.V.; MAVRIKAKIS, M.; Adsorption and Dissociation of $\mathrm{O}_{2}$ on $\mathrm{Pt}-\mathrm{Co}$ and Pt-Fe Alloys, Journal of the American Chemical Society, v.126, p.4717-4725, 2003.

45. BALBUENA, P. B.; ALTOMARE, D.; AGAPITO, L.; SEMINARIO, J. M., Theoretical Analysis of Oxygen Adsorption on Pt-Based Clusters Alloyed with Co, Ni, or Cr Embedded in a Pt Matrix, Journal of Physical Chemistry B, v.107, p.1367113680, 2003.

46. GOBAL, F.; ARAB, R.; NAHALI, M. A comparative DFT study of atomic and molecular oxygen adsorption on neutral and negatively charged $\operatorname{Pd}_{x} \mathrm{Cu}_{3-x}(x=0-3)$ nano-clusters. Journal of Molecular Structure: THEOCHEM, v.959, p. 15-21, 2010.

47. ANDERSON, A. B.; ALBU, T. V; Ab Initio Determination of Reversible Potentials and Activation Energies for Outer-Sphere Oxygen Reduction to Water and the Reverse Oxidation Reaction, Journal of the American Chemical Society, v.121, p. 11855-11863, 1999.

48.ZAGAL, J. H.; PAEZ, M.; TANAKA, A. A.; SANTOS JUNIOR, J. R.; LINKOUS, C., Journal of Electroanalytical Chemistry 339 (1-2)(1992) 13.

49. A. A. Tanaka, C. Fierro, D. A. Scherson, E. Yeager. Material Chemistry and Physics 22 (3-4)(1989) 431.

50.A. A. Tanaka, C. Fierro, D. Scherson, E. Yeager. Journal of Physical Chemistry, 91 (14)(1987) 3799.

51.ZAGAL, J.H., CARDENAS, G.I. Reactivity of immobilized cobalt phthalocyanines for the electroreduction of molecular oxygen in terms of molecular hardness, Journal of Electroanalytical Chemistry, v. 489, p. 96-100, 2000.

52. CARDENAS, G.I., GULPI, M.A., CARO, C.A., RIO, R., ZAGAL, J.H., Reactivity of electrodes modified with substituted metallophthalocyanines. Correlations with 
redox potentials, Hammett parameters and donor-acceptor intermolecular hardness, Electrochimica Acta, v. 46, p. 3227 - 3235, 2001.

53. CARDENAS, G.I., ZAGAL, J.H. Donor-acceptor intermolecular hardness on charge transfer reactions of substituted cobalt phthalocyanines, Journal of Electroanalytical Chemistry, v. 497, p.55-60, 2001.

54. ZAGAL, J.H., GULPI, M., ISAACAS, M., CARDENAS, G. AGUIRRE, M.J. Linear versus volcano correlations between electrocatalytic activity and redox and electronic properties of metallophthalocyanines, Electrochimica Acta, v. 44, p.1349-1357, 1998.

55. FRISCH, M. J.; TRUCKS, G. W.; SCHLEGEL, H. B.; SCUSERIA, G. E.; ROBB, M. A.; CHEESEMAN, J. R.; MONTGOMERY, J. A., JR.; VREVEN, T.; KUDIN, K. N.; BURANT, J. C.; MILLAM, J. M.; IYENGAR, S. S.; TOMASI, J.; BARONE, V.; MENNUCCI, B.; COSSI, M.; SCALMANI, G.; REGA, N.; PETERSSON, G. A.; NAKATSUJI, H.; HADA, M.; EHARA, M.; TOYOTA, K.; FUKUDA, R.; HASEGAWA, J.; ISHIDA, M.; NAKAJIMA, T.; HONDA, Y.; KITAO, O.; NAKAI, H.; KLENE, M.; LI, X.; KNOX, J. E.; HRATCHIAN, H. P.; CROSS, J. B.; ADAMO, C.; JARAMILLO, J.; GOMPERTS, R.; STRATMANN, R. E.; YAZYEV, O.; AUSTIN, A. J.; CAMMI, R.; POMELLI, C.; OCHTERSKI, J. W.; AYALA, P. Y.; MOROKUMA, K.; VOTH, G. A.; SALVADOR, P.; DANNENBERG, J. J.; ZAKRZEWSKI, V. G.; DAPPRICH, S.; DANIELS, A. D.; STRAIN, M. C.; FARKAS, O.; MALICK, D. K.; RABUCK, A. D.; RAGHAVACHARI, K.; FORESMAN, J. B.; ORTIZ, J. V.; CUI, Q.; BABOUL, A. G.; CLIFFORD, S.; CIOSLOWSKI, J.; STEFANOV, B. B.; LIU, G.; LIASHENKO, A.; PISKORZ, P.; KOMAROMI, I.; MARTIN, R. L.; FOX, D. J.; KEITH, T.; AL-LAHAM, M. A.; PENG, C. Y.; NANAYAKKARA, A.; CHALLACOMBE, M.; GILL, P.M. W.; JOHNSON, B.; CHEN, W.; WONG, M. W.; GONZALEZ, C.; POPLE, J. A. Gaussian 03, Revision B.04; Gaussian, Inc.: Pittsburgh PA, 2003.

56. POPLE, J.A.; HEAD-GORDON, M.; RAGHAVACHARI, K. J., Quadratic configuration interaction. A general technique for determining electron correlation energies, Journal of Chemical Physics, v.87, p.5968-5976, 1987. 
57. BECKE, A.D.; Density-functional thermochemistry. III. The role of exact exchange, Journal of Physical Chemistry, v.98, p.5648-5652, 1993.

58. GUPTA, S. K., NAPPI, B. M.; GRINGERICH, K. A., Mass spectrometric study of the stabilities of the gaseous molecules $\mathrm{Pt}_{2}$ and PtY, Inorganic Chemistry, v. 20, p. 966-973, 1981.

59. Li, T., BALBUENA, P.; Computational studies of the interaction of oxygen with platinum clusters, Journal of Physical Chemistry B, v.105, p.9943-9952, 2001.

60. TAYLOR, S.; LEMIRE, G. W.; HAMRICK, Y. M.; FU, Z.; MORSE, M. D., Resonant two-photon ionization spectroscopy of jet-cooled $\mathrm{Pt}_{2}$, Journal of Chemical Physics, v. 89, p. 5517, 1988.

61. HO, J.; POLAK, M. L.; ERVIN, K. M., LINEBERGER, W. C., Photoelectronspectroscopy of Nickel Group Dimers $\mathrm{Ni}^{2-}, \mathrm{Pd}^{2-}$ and $\mathrm{Pt}^{2-}$, Journal of Chemical Physics, v.99, p.8542-8551, 1993.

62. ERVIN, K. M.; HO, J.; LINEBERGER, W. C.; Electronic and vibrational structure of transition metal trimers: Photoelectron spectra of $\mathrm{Ni}_{3}{ }^{-}, \mathrm{Pd}_{3}{ }^{-}$, and $\mathrm{Pt}_{3}{ }^{-}$, Journal of Chemical Physics, v.89, p.4514-4519, 1988.

63. GANTEFOER, G.; GAUSE, G.; MEIWES-BROER, K.H.; LUTZ, H.O., Photoelectron Spectroscopy of Jet-cooled Aluminium Cluster Anions, Zeitschrift für Physik D: Atoms Molecules and Clusters, v.9, p.253-261, 1988.

64. STEARNS, C.A.; KOHL, F.J., Mass spectrometric determination of the dissociation energies of gaseous $\mathrm{Al}_{2}, \mathrm{AlSi}$, and $\mathrm{AlSiO}$, High Temperature Science, v.5, p.113-119, 1973. 
65. CASEY, S.M.; VILLATA, P.W.; BENGALI, A.A.; CHENG, C.-L.; DICK, J.P.; FENN, P.T.; LEOPOLD, D.G., A Study of $\mathrm{Cr} 2$ by Negative ion Photoelectron Spectroscopy, Journal of the American Chemical Society, v.113, p.6688-6694, 1991.

66. HILPERT, R. K., Determination of the dissociation energy of the $\mathrm{Cr}_{2}$ molecule, Berichte der Bunsengesellschaft für Physikalische Chemie, v.91, p.724-730, 1987.

67. DUNCAN, M. A.; KNIGTH, N, Y.; NAGAO, S.; JUDAI, K.; NAKAJIMA, A.; KAYA, K., Photoelectron spectroscopy of V-x(coronene)(y) and Ti- x(coronene)(y) anions, Journal of Physical Chemistry A, v. 105, p.10093-10097, 2001.

68. JAMES, A.M.; KOWALCZYK, P.; LANGLOIS, E.; CAMPBELL, M.D.; OGAWA, A.; SIMARD, B., Resonant two photon ionization spectroscopy of the molecules $V_{2}$, $\mathrm{VNb}$, and $\mathrm{Nb}_{2}$, Journal of Chemical Physics, v.101, p.4485-44, 1994.

69. STEININGER, H.; LEHWALD, S.; IBACH, H., Adsorption of Oxygen on Pt(111), Surface Science, v.123, p.1-17, 1982.

70. GLAND, J.; SEXTON, B. FISHER, G. B., Oxygen Interaction with Pt(111) Surface, Surface Science, v.95, p.587-602, 1980.

71. WURTH, W.; STOHR, J.; FEULNER, P.; PAN, X.; BAUCHSPIESS, K. R.; BABA, Y.; HUDEL, E.; HOCKER, G.; MENZEL, D., Bonding, structure, and magnetism of physisorbed and chemisorbed $\mathrm{O}_{2}$ on $\mathrm{Pt}(111)$, Physical Review Letters, v.65, p.2426-2429, 1990.

72. PUGLIA, C.; NILSSON, A.; HERNNAS, B.; KARIS, O.; BENNICH, P.; MARTENSSON, N., Physisorbed, Chemisorbed and dissociated $\mathrm{O}_{2}$ on $\mathrm{Pt}(111)$ studied by different core level spectroscopy methods, Surface Science, v.342, p.119-133, 1995. 
73. SIDIK, R. A.; ANDERSON, A. B., Density Functional Theory study of $\mathrm{O}_{2}$ electroreduction when bonded to a $\mathrm{Pt}$ dual site, Journal of Electroanalytical Chemistry, v.528, p.69-76, 2002. 